\title{
Peridynamics formulation for beam structures to predict damage in offshore structures
}

\author{
Cong Tien Nguyen ${ }^{\mathrm{a}, \mathrm{b}}$ and Selda Oterkus ${ }^{\mathrm{a},}$ \\ ${ }^{a}$ Department of Naval Architecture, Ocean and Marine Engineering, University of Strathclyde, \\ Glasgow, G11XQ, UK \\ ${ }^{\mathrm{b}}$ Faculty of Shipbuilding, Vietnam Maritime University, Haiphong, Vietnam
}

\begin{abstract}
Progressive damage analysis of structures is still a major challenge. Peridynamics is a non-local theory using the integral equations rather than differential equations which makes it suitable for damage prediction. In this study, a novel bond based peridynamic model is developed for threedimensional complex beam structures with 6 degrees of freedom based on Timoshenko beam theory. The numerical algorithm for dealing with complex beam structures is provided for the first time in the peridynamic literature. A damage criterion for beam structures with 6 degrees of freedom is also presented. The validity of peridynamic predictions is established by considering various examples. Initially, the proposed peridynamic model is used to predict the structural behavior of straight and curved beams. Next, the proposed peridynamic model is used to investigate a jacket platform. The jacket platform is subjected to both static and dynamic sea load induced by waves, winds, and currents. The peridynamic predictions are verified by comparing with finite element solutions. Peridynamic damage prediction for a pre-notched beam is verified by comparing with the previous literature. After verifying the peridynamic model, the proposed model is used to predict damages for a jacket platform due to sea loads and due to ship-jacket platform collisions.
\end{abstract}

Keywords: peridynamic beam model, damage, offshore structure, sea loads, collision

\section{Introduction}

Marine structures can experience damages although they are initially designed with high safety factors. These damages can be caused by many reasons such as collisions, groundings, explosions, corrosion, fatigue, overloading, etc. Predicting crack initiation and propagation is a challenging work that requires much effort. The equations in classical continuum mechanics are derived by using spatial derivatives. Therefore, traditional approaches face conceptual and mathematical difficulties in terms of predicting crack nucleation and growth, especially for multiple crack paths. This difficulty can be overcome by using peridynamics (PD) which is first introduced by Silling (2000). PD theory is based on integro-differential equations which are valid in both continuous and discontinuous models. Therefore, it is suitable to predict damages on structures such as crack propagation and multiple crack growths (Javili et al.,2018).

Peridynamics is applicable in both elastic and plastic material responses (Foster et al., 2010, Mitchell, 2011, Madenci and Oterkus, 2016a, 2017). Moreover, it can also be used to analyze composite and polycrystalline materials (Oterkus, 2010, Oterkus and Madenci, 2012, Hu et al., 2012, Gao and Oterkus, 2018, 2019, De Meo et al, 2016). Another advantage of peridynamics is that it can be applied for multiphysics (Oterkus,2015, Han et al.,2015, Madenci and Oterkus, 2016b, Oterkus et al.,2017) and multiscale modelling (Askari et al., 2008, Bobaru and Ha, 2011)

*Corresponding Author: Selda Oterkus, Department of Naval Architecture, Ocean and Marine Engineering, University of Strathclyde. Email: selda.oterkus@strath.ac.uk. 
PD can also be either combined with finite element analysis (FEA) (Kilic and Madenci, 2010b, Oterkus et al., 2012, Liu and Hong, 2012, Bie et al., 2018) or implemented in FEA framework (Macek and Silling, 2007, Han et al.,2016, Diyaroglu et al.,2017b, Yang et al., 2018).

Computational modeling of engineering structures in a three-dimensional (3D) fashion is usually computationally expensive. When analyzing complex structures, structural simplification can be used to reduce the computational cost. Thus, it is also essential to consider simplified peridynamic structural models. The first peridynamic model capturing tension and compression for $1 \mathrm{D}$ bars is introduced by Silling et al. (2003). Later, O'Grady and Foster (2014) introduced a non-ordinary state based PD model for Euler-Bernoulli beam by including transverse displacements but disregarding the transverse shear deformations. In order to include transverse shear deformations, Diyaroglu et al. (2015) introduced a bond based PD model based on Timoshenko beam theory which is applicable to both thin and moderately thick beams. The PD beam model provided by Diyaroglu et al. (2015) has two degrees of freedom which are transverse displacement and rotation. Moreover, Diyaroglu et al. (2017a) developed an ordinary state based PD model for EulerBernoulli beams. Recently, Yang et al. (2018) presented the implementation of PD beam and plate formulations in FEA framework.

It is obvious that a general beam has six degrees of freedom (DOF), three translational displacements, and three rotations. Moreover, beam structures in the real world often include many beams joined together, and each beam can be straight or curved. Therefore, this study focuses on developing six degrees of freedom PD beam model for complex beam type structures which are composed of straight and curved beams.

This paper is organized as follows. Section 2 describes the beam kinematics and strain energy density (SED) in classical continuum mechanics for beam structures. Section 3 provides PD formulation of SED, PD constants and PD equation of motion for beam structures. Section 4 describes the transformation of equation of motion from local to global coordinate system. Section 5 provides the damage criteria for beam structures based on critical energy release rate. Section 6 presents the numerical procedure for PD modelling of beam structures. In Section 7, static and dynamic analyses of the frame with straight beams, curved beam, and jacket platform are presented and the proposed PD model is first used to predict damage in a pre-notched beam then for a jacket platform. Finally, our conclusions are provided in Section 8.

\section{Beam kinematics in classical continuum mechanics}

In this section, first stress-strain relations for beam structures are obtained. Next, the strain energy density is established based on small deformation assumption. According to Timoshenko beam theory, it is assumed that plane cross-section remains plane after the deformation, but it does not have to remain normal to the neutral axis (Bathe, 2006). The Timoshenko beam has six degrees of freedom (Timoshenko et al., 1970); three displacements $(u, v, w)$ and three cross-sectional rotations $\left(\theta_{x}, \theta_{y}, \theta_{z}\right)$ as shown in Fig. 1 .

The displacement components of a material point can be defined as (Bathe, 2006)

$$
\begin{aligned}
& u(x, y, z)=\hat{u}(x)+z \theta_{y}(x)-y \theta_{z}(x) \\
& v(x, y, z)=\hat{v}(x)-z \theta_{x}(x)
\end{aligned}
$$


$w(x, y, z)=\hat{w}(x)+y \theta_{x}(x)$

where $x$ represents the beam's longitudinal axis which is located at the centreline of the beam and $\hat{u}, \hat{v}, \hat{w}$ represent the displacement components at the centreline of the beam.

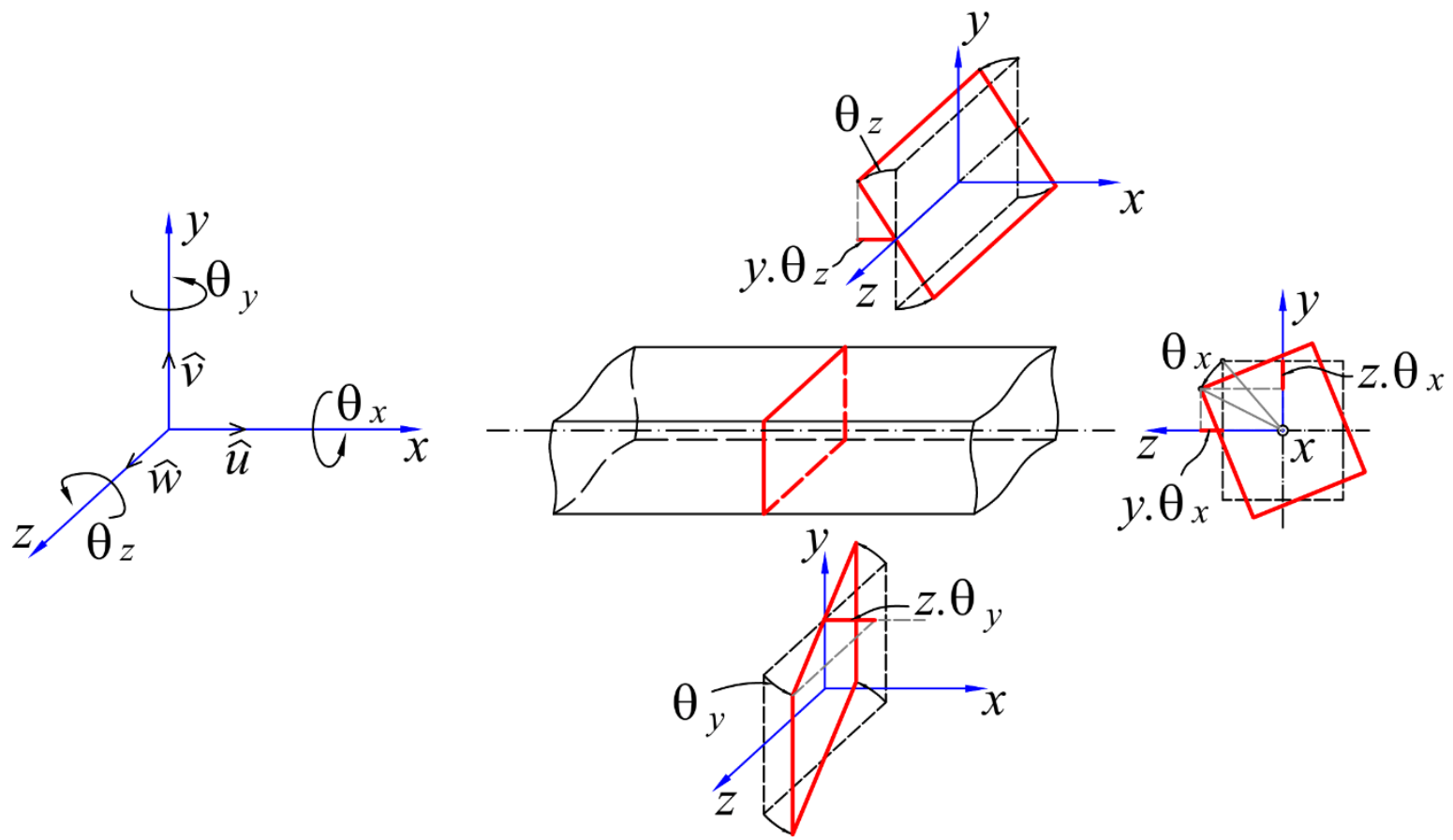

Fig. 1. Beam configuration with 6 DOFs

By using the displacement components defined in Eq. (1), the linear strain components of the beam can be calculated as

$$
\begin{aligned}
& \varepsilon_{x x}=u_{, x}=\hat{u}_{, x}+z \theta_{y, x}-y \theta_{z, x} \\
& \gamma_{x y}=u_{, y}+v_{, x}=\hat{v}_{, x}-\theta_{z}-z \theta_{x, x} \\
& \gamma_{x z}=u_{, z}+w_{, x}=\hat{w}_{, x}+\theta_{y}+y \theta_{x, x} \\
& \varepsilon_{y y}=\varepsilon_{z z}=\gamma_{y z}=0
\end{aligned}
$$

Based on small deformation assumption in linear elasticity, Cauchy stress components can be directly calculated from linear strain components as (Bathe, 2006)

$$
\begin{aligned}
& \sigma_{x x}=E \varepsilon_{x x}=E\left(\hat{u}_{, x}+z \theta_{y, x}-y \theta_{z, x}\right) \\
& \sigma_{x y}=G \gamma_{x y}=G\left(\hat{v}_{, x}-\theta_{z}-z \theta_{x, x}\right) \\
& \sigma_{x z}=G \gamma_{x z}=G\left(\hat{w}_{, x}+\theta_{y}+y \theta_{x, x}\right) \\
& \sigma_{y y}=\sigma_{z z}=\sigma_{y z}=0
\end{aligned}
$$

where $E$ represents the elastic modulus and $G$ represents the shear modulus. The strain energy per unit length of the beam can be calculated as

$U=\int_{A}\left[\frac{E}{2} \varepsilon_{x x}{ }^{2}+\frac{G}{2} \gamma_{x y}{ }^{2}+\frac{G}{2} \gamma_{x z}{ }^{2}\right] d A=U_{1}+U_{2}$

with 


$$
\begin{aligned}
& U_{1}=\frac{E}{2} \int_{A} \varepsilon_{x x}{ }^{2} d A \\
& U_{2}=\frac{G}{2} \int_{A}\left[\gamma_{x y}{ }^{2}+\gamma_{x z}{ }^{2}\right] d A
\end{aligned}
$$

where $A$ represents the cross-sectional area of the beam.

Utilizing strain definition given in Eq. (2), the strain energy per unit length of the beam given in Eq. (4) can be rewritten as

$$
\begin{aligned}
& U_{1}=\frac{E}{2} \int_{A}\left[\hat{u}_{, x}{ }^{2}+\left(z^{2} \cdot \theta_{y, x}{ }^{2}+y^{2} \cdot \theta_{z, x}{ }^{2}-2 y z \cdot \theta_{y, x} \theta_{z, x}\right)+2 \hat{u}_{, x}\left(z \cdot \theta_{y, x}-y \cdot \theta_{z, x}\right)\right] d A \\
& U_{2}=\frac{G}{2} \int_{A}\left[\begin{array}{l}
{\left[\left(\hat{v}_{, x}-\theta_{z}\right)^{2}+\left(\hat{w}_{, x}+\theta_{y}\right)^{2}\right]+\left(z^{2}+y^{2}\right) \theta_{x, x}{ }^{2}} \\
+\left(2 y\left(\hat{w}_{, x}+\theta_{y}\right)-2 z\left(\hat{v}_{, x}-\theta_{z}\right)\right) \theta_{x, x}
\end{array}\right] d A
\end{aligned}
$$

By considering the beam with a symmetric cross-section, the area moment of inertia and the first moment of area are obtained as

$$
\begin{aligned}
& I_{y y}=\int_{A} z^{2} d A ; I_{z z}=\int_{A} y^{2} d A ; I_{y z}=\int_{A} y z d A=0 \\
& S_{y}=\int_{A} z d A=0 ; S_{z}=\int_{A} y d A=0
\end{aligned}
$$

By utilizing relations given in Eq. (6), strain energy per unit length can be written as

$$
\begin{aligned}
& U_{1}=\frac{E A}{2} \hat{u}_{, x}{ }^{2}+\frac{E}{2}\left(I_{y y} \cdot \theta_{y, x}{ }^{2}+I_{z z} \cdot \theta_{z, x}{ }^{2}\right) \\
& U_{2}=\frac{G A}{2}\left[\left(\hat{v}_{, x}-\theta_{z}\right)^{2}+\left(\hat{w}_{, x}+\theta_{y}\right)^{2}\right]+\frac{G}{2}\left[\int_{A}\left(z^{2}+y^{2}\right) d A\right] \theta_{x, x}{ }^{2}
\end{aligned}
$$

By adding the shear correction factor, $k_{s}$ (Stephen, 1981), and replacing $\int_{A}\left(z^{2}+y^{2}\right) d A$ by torsional constant, $k_{t}$ (Boresi et al., 1985), Eq. (7b) becomes

$$
U_{2}=\frac{k_{s} G A}{2}\left[\left(\hat{v}_{, x}-\theta_{z}\right)^{2}+\left(\hat{w}_{, x}+\theta_{y}\right)^{2}\right]+\frac{k_{t} G}{2} \theta_{x, x}{ }^{2}
$$

By substituting Eq. (7a), (8) into Eq. (4a) the strain energy per unit length of the beam can be written as

$$
U=\frac{E A}{2} \hat{u}_{, x}^{2}+\frac{E}{2}\left(I_{y y} \cdot \theta_{y, x}{ }^{2}+I_{z z} \cdot \theta_{z, x}{ }^{2}\right)+\frac{k_{s} G A}{2}\left[\left(\hat{v}_{, x}-\theta_{z}\right)^{2}+\left(\hat{w}_{, x}+\theta_{y}\right)^{2}\right]+\frac{k_{t} G}{2} \theta_{x, x}{ }^{2}
$$

The strain energy density can be defined as

$$
W=\frac{U}{A}=\frac{E}{2} \hat{u}_{, x}{ }^{2}+\frac{E}{2 A}\left(I_{y y} \cdot \theta_{y, x}{ }^{2}+I_{z z} \cdot \theta_{z, x}{ }^{2}\right)+\frac{k_{s} G}{2}\left[\left(\hat{v}_{, x}-\theta_{z}\right)^{2}+\left(\hat{w}_{, x}+\theta_{y}\right)^{2}\right]+\frac{k_{t} G}{2 A} \theta_{x, x}{ }^{2}
$$

The strain energy density can also be decomposed into its axial, bending, shear and torsional components as

$W=W_{\text {axial }}+W_{\text {bending }}+W_{\text {shear }}+W_{\text {torsional }}$

where

$$
W_{\text {axial }}=\frac{E}{2} \hat{u}_{, x}^{2}
$$




$$
\begin{aligned}
& W_{\text {bending }}=\frac{E}{2 A}\left(I_{y y} \theta_{y, x}{ }^{2}+I_{z z} \theta_{z, x}{ }^{2}\right) \\
& W_{\text {shear }}=\frac{k_{s} G}{2}\left[\left(\hat{v}_{, x}-\theta_{z}\right)^{2}+\left(\hat{w}_{, x}+\theta_{y}\right)^{2}\right] \\
& W_{\text {torsional }}=\frac{k_{t} G}{2 A} \theta_{x, x}{ }^{2}
\end{aligned}
$$

\section{Beam kinematics in peridynamics}

Peridynamics is a new formulation of continuum theory which uses integro-differential equations. The peridynamic equation of motion for a material point is introduced by Silling (2000) and later generalized by Silling et al. (2007) as

$$
\rho(\mathbf{x}) \mathbf{u}(\mathbf{x}, t)=\int_{H_{\mathrm{x}}}\left(\mathbf{t}\left(\mathbf{u}^{\prime}-\mathbf{u}, \mathbf{x}^{\prime}-\mathbf{x}, t\right)-\mathbf{t}^{\prime}\left(\mathbf{u}-\mathbf{u}^{\prime}, \mathbf{x}-\mathbf{x}^{\prime}, t\right)\right) d H_{\mathrm{x}}+\mathbf{b}(\mathbf{x}, t)
$$

which can also be represented in discrete form as

$$
\rho_{(k)} \mathbf{u}_{(k)}=\sum_{j=1}^{N}\left(\mathbf{t}_{(k)(j)}\left(\mathbf{u}_{(j)}-\mathbf{u}_{(k)}, \mathbf{x}_{(j)}-\mathbf{x}_{(k)}, t\right)-\mathbf{t}_{(j)(k)}\left(\mathbf{u}_{(k)}-\mathbf{u}_{(j)}, \mathbf{x}_{(k)}-\mathbf{x}_{(j)}, t\right)\right) V_{(j)}+\mathbf{b}_{(k)}
$$

where $\rho$ represents the mass density, $\mathbf{u}$ represents the displacement vector and $\mathbf{b}$ represents the body force vector. In peridynamics, each material point has interactions with other material points within a distance which is called horizon size, $\delta$ as shown in Fig. 2. Material points within the horizon are called family members of material point $k$. In Eq. (12b) $N$ represents the number of family members of material point $k, j$ represents the family member material point $k, \mathbf{t}_{(k)(j)}$ represents the force density that material point $j$ exerts on material point $k$, and $\mathbf{t}_{(j)(k)}$ represents the force density that material point $k$ exerts on point $j$.

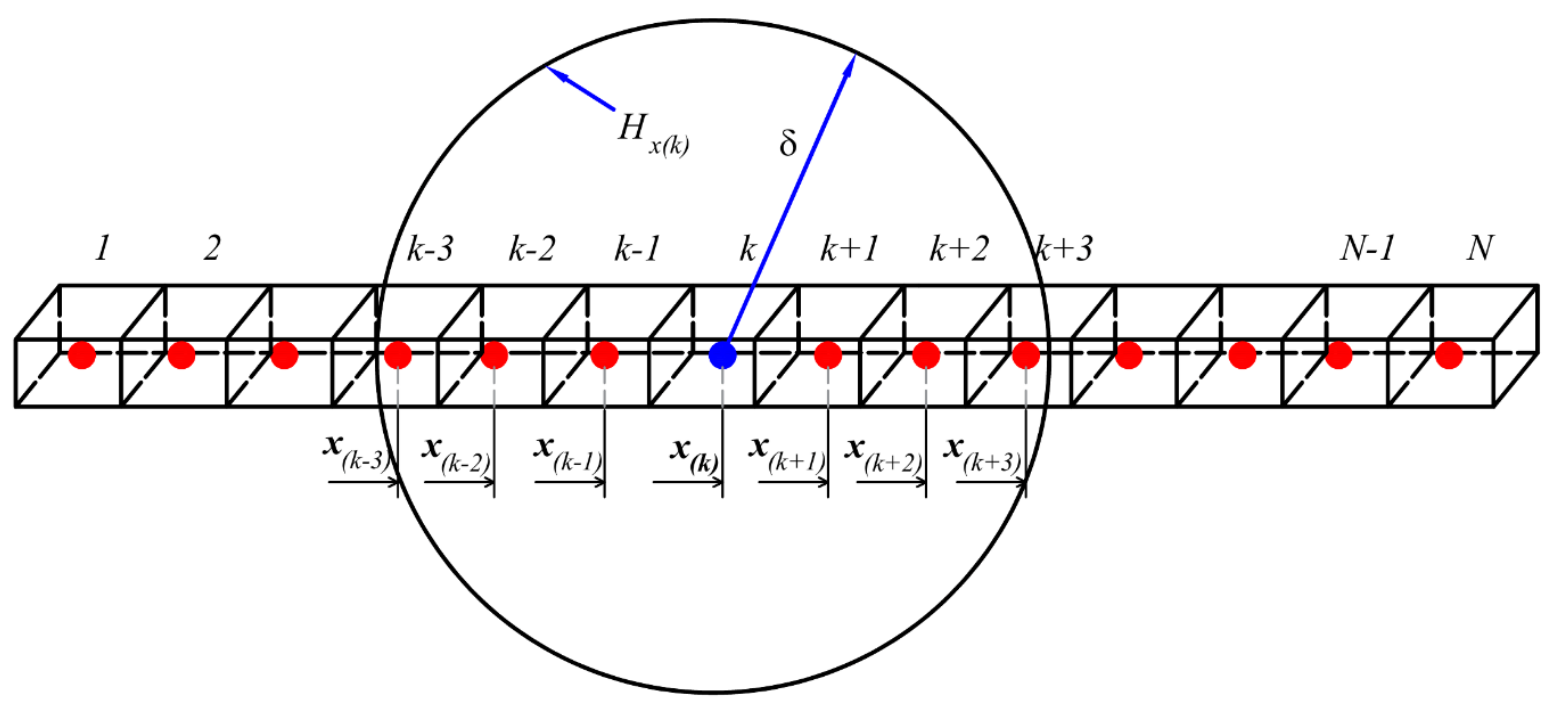

Fig. 2. PD material points and horizon size for a beam

As explained by Madenci and Oterkus (2014), the equation of motion can also be derived based on the principle of virtual work by satisfying the Lagrange's equation. Therefore, in Section 3.1, first, a PD form of strain energy is established and the PD constants are obtained by comparing 
SED in PD and classical continuum mechanics. After obtaining the Lagrangian function from kinetic energy and total potential energy, the equation of motion for the beam is obtained by using the Euler-Lagrange equation in Section 3.2.

\subsection{Strain energy density}

Similar to the SED in classical continuum mechanics provided in Eq. (11a), the SED for a beam in PD can also be represented as

$W_{(k)}^{P D}=W_{\text {axial }(k)}^{P D}+W_{\text {bending }(k)}^{P D}+W_{\text {shear }(k)}^{P D}+W_{\text {torsional }(k)}^{P D}$

Peridynamic form of axial part of SED was introduced by Diyaroglu (2016) as

$W_{\text {axial }(k)}^{P D}=\frac{1}{2} \sum_{j=1}^{N} \frac{1}{2} C_{a x}\left(\frac{u_{(j)}-u_{(k)}}{\xi}\right)^{2} \xi V_{(j)}$

In which, the bond constant $C_{a x}$ is defined as (Diyaroglu, 2016)

$$
C_{a x}=\frac{2 E}{A \delta^{2}}
$$

Here, $u_{(k)}$ and $u_{(j)}$ represent the axial displacement of material point $k$ and $j$, respectively. The parameter $\xi$ represents the distance between two material points $k$ and $j$.

Peridynamic form of bending part of SED in Eq. (13) can be expressed as

$$
W_{b e n d i n g(k)}^{P D}=\frac{1}{2} \sum_{j=1}^{N} \frac{1}{2}\left[C_{b y}\left(\frac{\theta_{y(j)}-\theta_{y(k)}}{\xi}\right)^{2}+C_{b z}\left(\frac{\theta_{z(j)}-\theta_{z(k)}}{\xi}\right)^{2}\right] \xi V_{(j)}
$$

where $C_{b y}$ and $C_{b z}$ represent the bending bond constants which can be determined as follows.

First, the rotational angles of material point $j$ is expressed by using the first two terms in Taylor's series expansion as

$$
\begin{aligned}
& \theta_{y(j)}=\theta_{y(k)}+\theta_{y(k), x}\left(x_{(j)}-x_{(k)}\right) \\
& \theta_{z(j)}=\theta_{z(k)}+\theta_{z(k), x}\left(x_{(j)}-x_{(k)}\right)
\end{aligned}
$$

or

$\frac{\theta_{y(j)}-\theta_{y(k)}}{\xi}=\theta_{y(k), x} \operatorname{sgn}\left(x_{(j)}-x_{(k)}\right)$

$\frac{\theta_{z(j)}-\theta_{z(k)}}{\xi}=\theta_{z(k), x} \operatorname{sgn}\left(x_{(j)}-x_{(k)}\right)$

with

$\operatorname{sgn}\left(x_{(j)}-x_{(k)}\right)=\frac{x_{(j)}-x_{(k)}}{\xi}$

By using the relations in Eq.(16), the PD form of bending part of SED in Eq. (15) can be written as

$$
W_{\text {bending }(k)}^{P D}=\frac{1}{2} \sum_{j=1}^{N} \frac{1}{2}\left[C_{b y}\left(\theta_{y, x} \operatorname{sgn}\left(x_{(j)}-x_{(k)}\right)\right)^{2}+C_{b z}\left(\theta_{y, x} \operatorname{sgn}\left(x_{(j)}-x_{(k)}\right)\right)^{2}\right] \xi V_{(j)}
$$

or 


$$
W_{b e n d i n g(k)}^{P D}=\frac{1}{2} \sum_{j=1}^{N} \frac{1}{2}\left[C_{b y}\left(\theta_{y, x}\right)^{2}+C_{b z}\left(\theta_{y, x}\right)^{2}\right] \xi V_{(j)}
$$

Next, bending strain energy density given in Eq. (17b) can be rewritten in the integral form by disregarding the peridynamic interactions beyond the horizon as

$$
W_{\text {bending }}^{P D}(x)=2 A \int_{0}^{\delta} \frac{1}{4}\left[C_{b y}\left(\theta_{y, x}\right)^{2}+C_{b z}\left(\theta_{y, x}\right)^{2}\right] \xi d \xi
$$

After performing the integrations in Eq. (18), the strain energy density for bending part becomes

$$
W_{\text {bending }}^{P D}(x)=\frac{1}{4} A \delta^{2}\left[C_{b y} \theta_{y, x}{ }^{2}+C_{b z} \theta_{z, x}{ }^{2}\right]
$$

By comparing SED given in Eq. (19) and Eq. (11c), the PD constants for bending can be determined as

$$
\begin{aligned}
C_{b y} & =\frac{2 E I_{y y}}{A^{2} \delta^{2}} \\
C_{b z} & =\frac{2 E I_{z z}}{A^{2} \delta^{2}}
\end{aligned}
$$

Peridynamic form of shear part of SED in Eq. (13) can be expressed as

$$
W_{\text {shear }(k)}^{P D}=\frac{1}{2} \sum_{j=1}^{N} \frac{1}{2} C_{s}\left[\begin{array}{l}
\left(\frac{v_{(j)}-v_{(k)}}{\xi} \operatorname{sgn}\left(x_{(j)}-x_{(k)}\right)-\frac{\theta_{z(j)}+\theta_{z(k)}}{2}\right)^{2} \\
+\left(\frac{w_{(j)}-w_{(k)}}{\xi} \operatorname{sgn}\left(x_{(j)}-x_{(k)}\right)+\frac{\theta_{y(j)}+\theta_{y(k)}}{2}\right)^{2}
\end{array}\right] \xi V_{(j)}
$$

where $C_{s}$ represents the shear bond constant which can be determined as follows.

First, similar to the bending part, the explicit expression of shear SED in Eq. (21) can be written in integral form as

$$
W_{\text {shear }(k)}^{P D}=2 A C_{s} \frac{1}{4} \int_{0}^{\delta}\left[\left(v_{, x}-\theta_{z}\right)^{2}+\left(w_{, x}+\theta_{y}\right)^{2}\right] \xi d \xi
$$

Next, by performing integrations in Eq. (22), the peridynamic form of shear part of SED can be rewritten as

$$
W_{s}^{P D}=\frac{1}{4} A \delta^{2} C_{s}\left[\left(v_{, x}-\theta_{z}\right)^{2}+\left(w_{, x}+\theta_{y}\right)^{2}\right]
$$

By comparing Eq. (23) and Eq. (11d), the PD constant for shear part of SED can be defined as

$$
C_{s}=\frac{2 k_{s} G}{A \delta^{2}}
$$

Peridynamic form of torsional part of SED in Eq. (13) can be explicitly expressed according to Diyaroglu (2016) as

$$
W_{\text {torsional }(k)}^{P D}=\frac{1}{2} \sum_{j=1} C_{t} \frac{1}{2}\left(\frac{\theta_{x(j)}-\theta_{x(k)}}{\xi}\right)^{2} \xi V_{(j)}
$$

In which, the torsional bond constant $C_{t}$ can be defined as (Diyaroglu, 2016)

$$
C_{t}=\frac{2 k_{t} G}{A^{2} \delta^{2}}
$$




\subsection{Equations of motion}

The equations of motion for a beam can be achieved by using the Euler-Lagrange equation (Madenci and Oterkus, 2014). The Euler-Lagrange equation can be written as

$\frac{d}{d t}\left(\frac{\partial L}{\partial q_{i}}\right)-\frac{\partial L}{\partial q_{i}}=0$

where $L$ represents the Lagrangian, $q_{i}$ represents the degree of freedom with $\mathbf{q}=\left\{\begin{array}{llllll}u & v & w & \theta_{x} & \theta_{y} & \theta_{z}\end{array}\right\}$ and $q_{i}$ represents the time derivative of $q_{i}$.

The Lagrangian function for a beam can be expressed as

$$
L=T-U
$$

with

$$
\begin{aligned}
& T=\frac{\rho}{2} \sum_{k=1}^{N}\left(u_{(k)}^{2}+v_{(k)}^{2}+w_{(k)}^{2}+\frac{I_{x x}}{A} \theta_{x(k)}^{2}+\frac{I_{y y}}{A} \theta_{y(k)}^{2}+\frac{I_{z z}}{A} \theta_{z(k)}^{2}\right) V_{(k)} \\
& U=\sum_{k=1}^{N}\left(\begin{array}{c}
W_{(k)}^{P D}-b_{x(k)} u_{(k)}-b_{y(k)} v_{(k)}-b_{z(k)} w_{(k)} \\
-m_{x(k)} \theta_{x(k)}-m_{y(k)} \theta_{y(k)}-m_{z(k)} \theta_{z(k)}
\end{array}\right) V_{(k)}
\end{aligned}
$$

where $T$ represents the total kinetic energy, $U$ represents the total potential energy, $b_{x(k)}, b_{y(k)}$, $b_{z(k)}$ represent the applied body forces, $m_{x(k)}, m_{y(k)}, m_{z(k)}$ represent the moment per unit volume at material point $k$. Substituting Lagrangian provided in Eq. (27) into Eq. (26), the peridynamic form of the equation of motion for a beam can be obtained as

$$
\begin{aligned}
& \rho u_{(k)}=C_{a x} \sum_{j=1}^{N}\left(\frac{u_{(j)}-u_{(k)}}{\xi}\right) V_{(j)}+b_{x(k)} \\
& \rho v_{(k)}=C_{s} \sum_{j=1}^{N}\left(\frac{v_{(j)}-v_{(k)}}{\xi}-\frac{\theta_{z(j)}+\theta_{z(k)}}{2} \operatorname{sgn}\left(x_{(j)}-x_{(k)}\right)\right) V_{(j)}+b_{y(k)} \\
& \rho w_{(k)}=C_{s} \sum_{j=1}^{N}\left(\frac{w_{(j)}-w_{(k)}}{\xi}+\frac{\theta_{y(j)}+\theta_{y(k)}}{2} \operatorname{sgn}\left(x_{(j)}-x_{(k)}\right)\right) V_{(j)}+b_{z(k)} \\
& \frac{\rho I_{x x}}{A} \theta_{x(k)}=C_{t} \sum_{j=1}^{N}\left(\frac{\left.\theta_{x(j)}-\theta_{x(k)}\right)}{\xi}\right) V_{(j)}+m_{x(k)} \\
& \frac{\rho I_{y y}}{A} \theta_{y(k)}=C_{b y} \sum_{j=1}^{N}\left(\frac{\theta_{y(j)}-\theta_{y(k)}}{\xi}\right) V_{(j)} \\
& \quad-\frac{1}{2} C_{s} \sum_{j=1}^{N}\left(\frac{w_{(j)}-w_{(k)}}{\xi} \operatorname{sgn}\left(x_{(j)}-x_{(k)}\right)+\frac{\theta_{y(j)}+\theta_{y(k)}}{2}\right) \xi V_{(j)}+m_{y(k)} \\
& \frac{\rho I_{z z}}{A} \theta_{z(k)}=C_{b z} \sum_{j=1}^{N}\left(\frac{\left.\theta_{z(j)}-\theta_{z(k)}\right)}{\xi}\right) V_{(j)} \\
& +\frac{1}{2} C_{s} \sum_{j=1}^{N}\left(\frac{v_{(j)}-v_{(k)}}{\xi} \operatorname{sgn}\left(x_{(j)}-x_{(k)}\right)-\frac{\theta_{z(j)}+\theta_{z(k)}}{2}\right) \xi V_{(j)}+m_{z(k)}
\end{aligned}
$$

The PD form of the equation of motion given in Eq. (28) can also be written in the vector form as 
$\mathbf{m}_{(k)} \mathbf{u}_{(k)}=\sum_{j=1}^{N} \mathbf{f}_{(k)(j)} V_{(j)}+\mathbf{b}_{(k)}$

where

$\mathbf{m}_{(k)}=\left[\begin{array}{cccccc}\rho & 0 & 0 & 0 & 0 & 0 \\ 0 & \rho & 0 & 0 & 0 & 0 \\ 0 & 0 & \rho & 0 & 0 & 0 \\ 0 & 0 & 0 & \frac{\rho I_{x x}}{A} & 0 & 0 \\ 0 & 0 & 0 & 0 & \frac{\rho I_{y y}}{A} & 0 \\ 0 & 0 & 0 & 0 & 0 & \frac{\rho I_{z z}}{A}\end{array}\right] ; \mathbf{u}_{(k)}=\left[\begin{array}{c}u_{(k)} \\ v_{(k)} \\ w_{(k)} \\ \theta_{x(k)} \\ \theta_{y(k)} \\ \theta_{z(k)}\end{array}\right] ; \mathbf{b}_{(k)}=\left[\begin{array}{c}b_{x(k)} \\ b_{y(k)} \\ b_{z(k)} \\ m_{x(k)} \\ m_{y(k)} \\ m_{z(k)}\end{array}\right]$

and

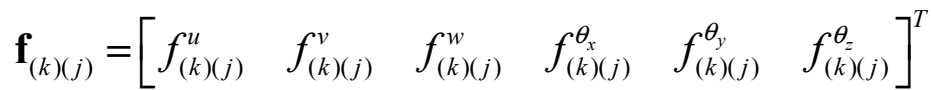

with

$$
\begin{aligned}
& f_{(k)(j)}^{u}=C_{a x}\left(\frac{u_{(j)}-u_{(k)}}{\xi}\right) \\
& f_{(k)(j)}^{v}=C_{s}\left(\frac{v_{(j)}-v_{(k)}}{\xi}-\frac{\theta_{z(j)}+\theta_{z(k)}}{2} \operatorname{sgn}\left(x_{(j)}-x_{(k)}\right)\right) \\
& f_{(k)(j)}^{w}=C_{s}\left(\frac{w_{(j)}-w_{(k)}}{\xi}+\frac{\theta_{y(j)}+\theta_{y(k)}}{2} \operatorname{sgn}\left(x_{(j)}-x_{(k)}\right)\right) \\
& f_{(k)(j)}^{\theta_{x}}=C_{t}\left(\frac{\theta_{x(j)}-\theta_{x(k)}}{\xi}\right) \\
& f_{(k)(j)}^{\theta_{y}}=C_{b y}\left(\frac{\theta_{y(j)}-\theta_{y(k)}}{\xi}\right)-\frac{1}{2} C_{s}\left(\frac{w_{(j)}-w_{(k)}}{\xi} \operatorname{sgn}\left(x_{(j)}-x_{(k)}\right)+\frac{\theta_{y(j)}+\theta_{y(k)}}{2}\right) \xi \\
& f_{(k)(j)}^{\theta_{z}}=C_{b z}\left(\frac{\theta_{z(j)}-\theta_{z(k)}}{\xi}\right)+\frac{1}{2} C_{s}\left(\frac{v_{(j)}-v_{(k)}}{\xi} \operatorname{sgn}\left(x_{(j)}-x_{(k)}\right)-\frac{\theta_{z(j)}+\theta_{z(k)}}{2}\right) \xi
\end{aligned}
$$

\section{Coordinate systems and transformation of equations of motion}

The equations of motion provided in Eq. (28) are derived in the local coordinate system. However, geometrical properties of complex structures are often governed with respect to the global coordinate system. Therefore, the equation of motion for each material point needs to be transformed from local to global coordinates. In this section, first, the relationship between local and global coordinates is provided in Section 4.1. Next, the transformation of equation of motion for straight and curved beams are presented in Sections 4.2 and 4.3, respectively.

\subsection{Local and global coordinate systems}

In the global coordinate system, the orientation of each beam is governed by the unit vectors, $n_{x}$, 
$n_{y}, n_{z}$ located at the centreline of the beam as shown in Fig. 3. If the beam is straight, every crosssection of the beam has same unit vectors. On the other hand, if the beam is curved, the unit vectors for each section will be different as shown in Fig. 3. Assuming that the unit vectors of material point $k$ are defined as

$$
\begin{aligned}
& n_{x(k)}=\left[\begin{array}{lll}
a_{1(k)} & a_{2(k)} & a_{3(k)}
\end{array}\right]^{T} \\
& n_{y(k)}=\left[\begin{array}{lll}
b_{1(k)} & b_{2(k)} & b_{3(k)}
\end{array}\right]^{T} \\
& n_{z(k)}=\left[\begin{array}{lll}
c_{1(k)} & c_{2(k)} & c_{3(k)}
\end{array}\right]^{T}
\end{aligned}
$$

Straight beam

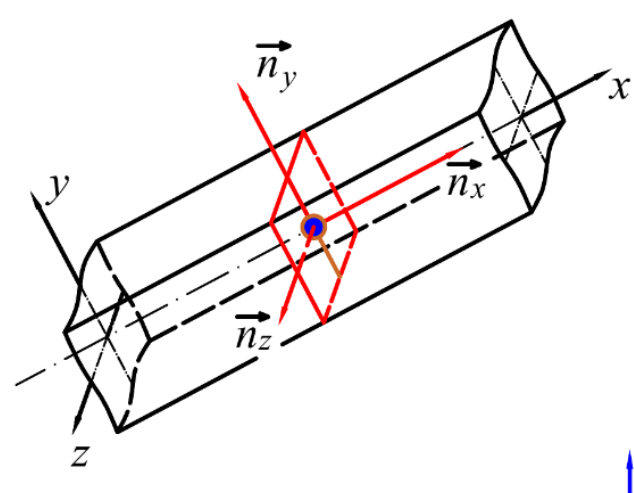

\section{Curved beam}

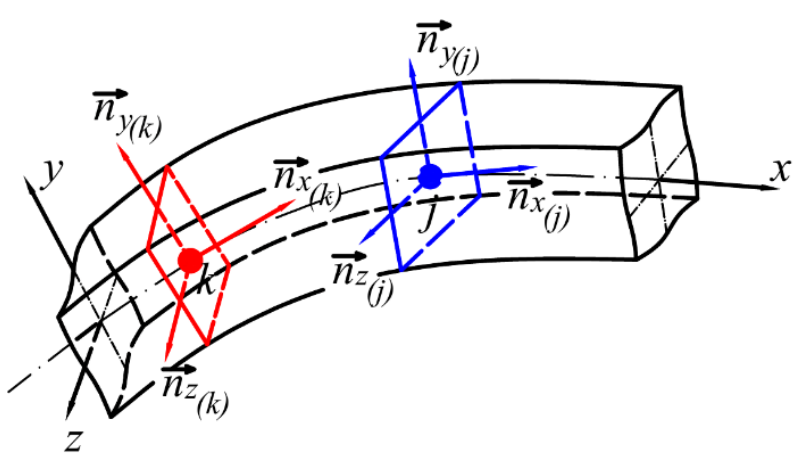

Fig. 3. Local and global coordinate systems

The relationship between global and local coordinates can be defined as

$\left[\begin{array}{c}x_{(k)} \\ y_{(k)} \\ z_{(k)}\end{array}\right]=\left[\begin{array}{lll}a_{1(k)} & a_{2(k)} & a_{3(k)} \\ b_{1(k)} & b_{2(k)} & b_{3(k)} \\ c_{1(k)} & c_{2(k)} & b_{3(k)}\end{array}\right]\left[\begin{array}{c}x_{(k)}^{\prime} \\ y_{(k)}^{\prime} \\ z_{(k)}^{\prime}\end{array}\right]=\mathbf{H}_{(k)}\left[\begin{array}{c}x_{(k)}^{\prime} \\ y_{(k)}^{\prime} \\ z_{(k)}^{\prime}\end{array}\right]$

with

$\mathbf{H}_{(k)}=\left[\begin{array}{lll}n_{x(k)} & n_{y(k)} & n_{z(k)}\end{array}\right]^{T}$

where $\mathbf{H}_{(k)}$ represents coordinate transformation matrix of material point $k$. The transformation of displacement vectors can be also defined as

$\mathbf{u}_{(k)}=\mathbf{T}_{(k)} \mathbf{u}_{(k)}^{\prime}$

with

$\mathbf{u}_{(k)}=\left[\begin{array}{llllll}u_{(k)} & v_{(k)} & w_{(k)} & \theta_{x(k)} & \theta_{y(k)} & \theta_{z(k)}\end{array}\right]^{T}$

$\mathbf{u}_{(k)}^{\prime}=\left[\begin{array}{llllll}u_{(k)}^{\prime} & v_{(k)}^{\prime} & w_{(k)}^{\prime} & \theta_{x^{\prime}(k)} & \theta_{y^{\prime}(k)} & \theta_{z^{\prime}(k)}\end{array}\right]^{T}$

where $\mathbf{u}_{(k)}$ and $\mathbf{u}_{(k)}^{\prime}$ are displacement vectors in the local and global coordinate systems, 
respectively. $\mathbf{T}_{(k)}$ represents the displacement transformation matrix of material point $k$. This matrix can be obtained from the coordinate transformation matrix, $\mathbf{H}_{(k)}$, as

$$
\mathbf{T}_{(k)}=\left[\begin{array}{cc}
\mathbf{H}_{(k)} & \mathbf{0} \\
\mathbf{0} & \mathbf{H}_{(k)}
\end{array}\right]
$$

\subsection{Transformation of equations of motion for straight beams}

By using the relations given in Eq. (32a), the equations of motion for a beam given in Eq. (29a) can be written as

$\mathbf{m}_{(k)} \mathbf{T}_{(k)} \mathbf{u}_{(k)}^{\prime}=\sum_{j=1}^{N} \mathbf{f}_{(k)(j)} V_{(j)}+\mathbf{b}_{(k)}$

Multiplying both sides by $\mathbf{T}_{(k)}^{T}$ results in

$\mathbf{m}_{(k)}^{\prime} \mathbf{u}_{(k)}^{\prime}=\mathbf{T}_{(k)}^{T}\left(\sum_{j=1}^{N} \mathbf{f}_{(k)(j)} V_{(j)}+\mathbf{b}_{(k)}\right)$

As a result, the equation of motion in global coordinates becomes

$\mathbf{m}_{(k)}^{\prime} \mathbf{u}_{(k)}^{\prime}=\sum_{j=1}^{N} \mathbf{f}_{(k)(j)}^{\prime} V_{(j)}+\mathbf{b}_{(k)}^{\prime}$

where

$\mathbf{f}_{(k)(j)}^{\prime}=\mathbf{T}_{(k)}^{T} \mathbf{f}_{(k)(j)}$

$\mathbf{b}_{(k)}^{\prime}=\mathbf{T}_{(k)}^{T} \mathbf{b}_{(k)}$

$\mathbf{m}_{(k)}^{\prime}=\mathbf{T}_{(k)}^{T} \mathbf{m}_{(k)} \mathbf{T}_{(k)}$

Note that $\operatorname{sgn}\left(x_{(j)}-x_{(k)}\right)$ in Eq. (17c) can be calculated in global coordinates by using Eq. (31) as $\operatorname{sgn}\left(x_{(j)}-x_{(k)}\right)=\frac{\left(a_{1(j)} x_{(j)}^{\prime}+a_{2(j)} y_{(j)}^{\prime}+a_{3(j)} z_{(j)}^{\prime}\right)-\left(a_{1(k)} x_{(k)}^{\prime}+a_{2(k)} y_{(k)}^{\prime}+a_{3(k)} z_{(k)}^{\prime}\right)}{\xi}$

4.3. Transformation of equations of motion for curved beams

The equations of motion for a curved beam can be obtained by modifying Eq. (35) with a new transformation matrix as

$\mathbf{m}_{(k)}^{\prime} \mathbf{u}_{(k)}^{\prime}=\mathbf{T}_{(k)(j)}^{T}\left(\sum_{j=1}^{N} \mathbf{f}_{(k)(j)} V_{(j)}+\mathbf{b}_{(k)}\right)$

with

$\mathbf{m}_{(k)}^{\prime}=\mathbf{T}_{(k)(j)}^{T} \mathbf{m}_{(k)} \mathbf{T}_{(k)(j)}$

where the new transformation matrix can be defined as

$\mathbf{T}_{(k)(j)}=\left[\begin{array}{cc}\mathbf{H}_{(k)(j)} & \mathbf{0} \\ \mathbf{0} & \mathbf{H}_{(k)(j)}\end{array}\right]$

with

$\mathbf{H}_{(k)(j)}=\left[\begin{array}{lll}n_{x(k)(j)} & n_{y(k)(j)} & n_{z(k)(j)}\end{array}\right]^{T}$

where $n_{x(k)(j)}, n_{y(k)(j)}, n_{z(k)(j)}$ can be represented as 


$$
\begin{aligned}
& n_{x(k)(j)}=\frac{\left(n_{x(k)}+n_{x(j)}\right)}{\left|\left(n_{x(k)}+n_{x(j)}\right)\right|} \\
& n_{y(k)(j)}=\frac{\left(n_{y(k)}+n_{y(j)}\right)}{\left|\left(n_{y(k)}+n_{y(j)}\right)\right|} \\
& n_{z(k)(j)}=\frac{\left(n_{z(k)}+n_{z(j)}\right)}{\left|\left(n_{z(k)}+n_{z(j)}\right)\right|}
\end{aligned}
$$

\section{Damage prediction in peridynamics}

A damage process in peridynamics is introduced through the elimination of interactions between material points. When the interaction between two material points is broken, the force densities between these points are irreversibly removed, and it leads to crack growth. Considering an interaction between material points $k$ and $j$, the state of interaction can be represented by a function, $\mu_{(k)(j)}$ which is introduced by Silling and Askari (2005) as

$\mu_{(k)(j)}\left(\mathbf{x}_{(j)}-\mathbf{x}_{(k)}, t\right)= \begin{cases}1 & \text { if interaction exists, } \\ 0 & \text { if interaction is broken }\end{cases}$

Therefore, the PD equation of motion given in Eq. (36) can be written as

$\mathbf{m}_{(k)}^{\prime} \mathbf{u}_{(k)}^{\prime}=\sum_{j=1}^{N} \mu_{(k)(j)} \mathbf{f}_{(k)(j)}^{\prime} V_{(j)}+\mathbf{b}_{(k)}^{\prime}$

The local damage, $\varphi\left(\mathbf{x}_{(k)}, t\right)$, which is the ratio of eliminated interactions to the total number of interactions associated with a material point within its horizon can be represented as (Silling and Askari, 2005)

$\varphi\left(\mathbf{x}_{(k)}, t\right)=1-\frac{\int_{H} \mu_{(k)(j)}\left(\mathbf{x}_{(j)}-\mathbf{x}_{(k)}, t\right) d V_{(j)}}{\int_{H} d V_{(j)}}$

The value of the local damage is between 0 and 1 , in which $\varphi=0$ represents no damage at the material point, and $\varphi=1$ represents all interactions of that material point with others within its horizon are eliminated. Moreover, $\varphi=0.5$ represents half of the interactions are eliminated with the related material point.

The damage criteria for each interaction can be represented by either critical bond stretch (Silling, 2000) or critical energy release rate (Madenci and Oterkus, 2016, 2017). In this study, the damage criteria based on the critical energy release rate is chosen. The critical energy release rate, $g_{c}$, for one interaction can be obtained by the relation suggested by Madenci and Oterkus $(2016,2017)$ as $g_{c}=\frac{G_{c}}{N_{c}}$

where $G_{c}$ represents the critical energy release rate for a material, and $N_{c}$ represents the total number of interactions passing through a unit crack surface, $A$. This number can be defined by counting the interactions passing through a unit crack surface. As shown in Fig. 4, there are 
$N_{c}=12$ interactions passing through the crack surface.
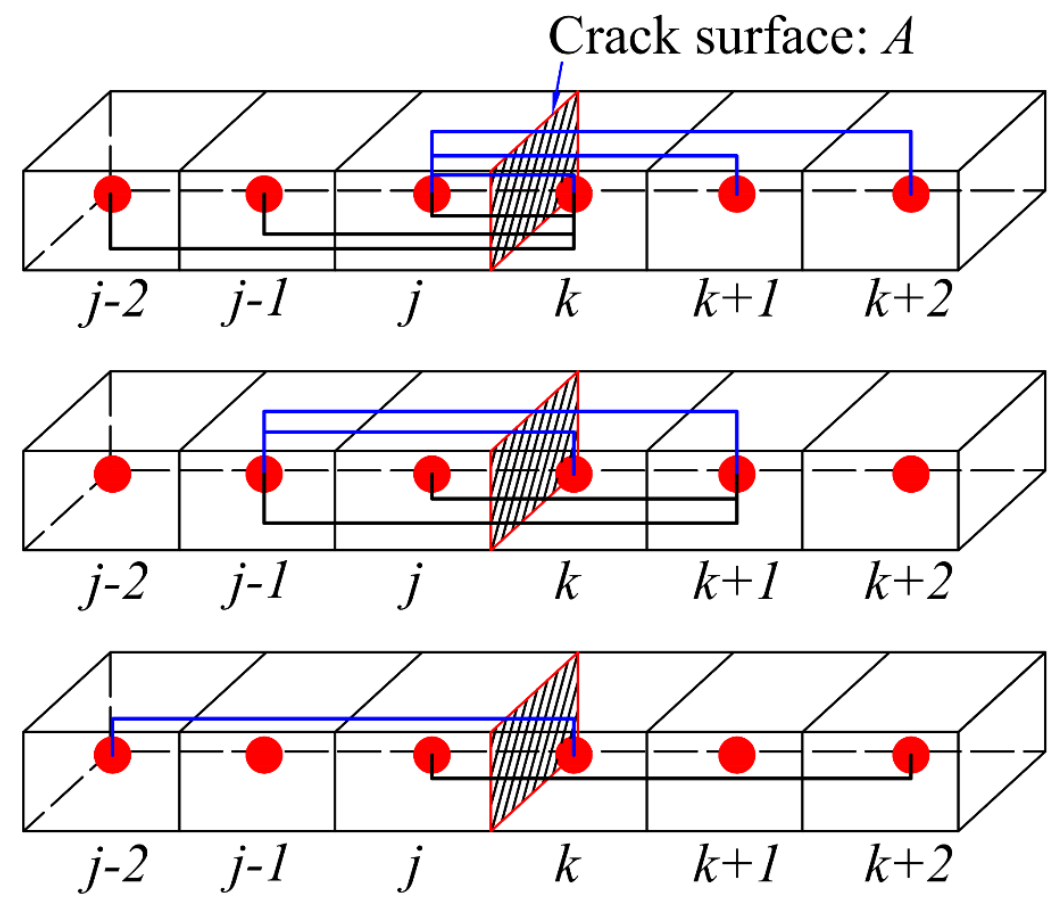

Fig. 4. Counting number of interaction passing through the crack surface $A$

The damage criteria in peridynamics for a beam can be described as

$\bar{g}_{(k)(j)}<g_{c} \rightarrow$ interaction exists: $\mu_{(k)(j)}=1$

$\bar{g}_{(k)(j)} \geq g_{c} \rightarrow$ interaction is broken: $\mu_{(k)(j)}=0$

where $\bar{g}_{(k)(j)}$ represents the energy release rate for interaction between material points $k$ and $j$ which can be calculated as

$\bar{g}_{(k)(j)}=\frac{1}{2}\left(g_{(k)(j)}+g_{(j)(k)}\right)$

with

$g_{(k)(j)}=\frac{1}{A} \Phi_{(k)(j)} V_{(k)} V_{(j)}$
$g_{(j)(k)}=\frac{1}{A} \Phi_{(j)(k)} V_{(j)} V_{(k)}$

where $\Phi_{(k)(j)}$ and $\Phi_{(j)(k)}$ represent micropotentials of the interaction between material point $k$ and $j$. By applying the same idea introduced by Madenci and Oterkus $(2016,2017)$, the micropotential $\Phi_{(k)(j)}$ for one interaction can be calculated as 


$$
\begin{aligned}
\Phi_{(k)(j)} & =\Phi_{(k)(j)}^{u}+\Phi_{(k)(j)}^{v}+\Phi_{(k)(j)}^{w}+\Phi_{(k)(j)}^{\theta_{x}}+\Phi_{(k)(j)}^{\theta_{y}}+\Phi_{(k)(j)}^{\theta_{z}} \\
& =\int_{0}^{s_{(k)(j)}^{u}} t_{(k)(j)}^{u} \xi d s_{(k)(j)}^{u}+\int_{0}^{s_{(k)(j)}^{v}} t_{(k)(j)}^{v} \xi d s_{(k)(j)}^{v}+\int_{0}^{s_{(k)(j)}^{w}} t_{(k)(j)}^{w} \xi d s_{(k)(j)}^{w} \\
& +\int_{0}^{s_{(k)(j)}^{\theta_{x}}} t_{(k)(j)}^{\theta_{x}} \xi d s_{(k)(j)}^{\theta_{x}}+\int_{0}^{s_{(k)(j)}^{\theta_{y}}} t_{(k)(j)}^{\theta_{y}} \xi d s_{(k)(j)}^{\theta_{y}}+\int_{0}^{s_{(k)(j)}^{\theta_{z}}} t_{(k)(j)}^{\theta_{z}} \xi d s_{(k)(j)}^{\theta_{z}}
\end{aligned}
$$

As shown in Fig. 5, the micropotential $\Phi_{(k)(j)}$ given in Eq. (48) can be simplified for linear analysis as

$$
\begin{aligned}
& \Phi_{(k)(j)}=\frac{1}{2} t_{(k)(j)}^{u} \xi s_{(k)(j)}^{u}+\frac{1}{2} t_{(k)(j)}^{v} \xi s_{(k)(j)}^{v}+\frac{1}{2} t_{(k)(j)}^{w} \xi s_{(k)(j)}^{w} \\
& +\frac{1}{2} t_{(k)(j)}^{\theta_{x}} \xi s_{(k)(j)}^{\theta_{x}}+\frac{1}{2} t_{(k)(j)}^{\theta_{y}} \xi s_{(k)(j)}^{\theta_{y}}+\frac{1}{2} t_{(k)(j)}^{\theta_{z}} \xi s_{(k)(j)}^{\theta_{z}}
\end{aligned}
$$

where

$$
\begin{aligned}
& s_{(k)(j)}^{u}=\frac{u_{(j)}-u_{(k)}}{\xi}, t_{(k)(j)}^{u}=\frac{1}{2} f_{(k)(j)}^{u} \\
& s_{(k)(j)}^{v}=\frac{v_{(j)}-v_{(k)}}{\xi}, t_{(k)(j)}^{v}=\frac{1}{2} f_{(k)(j)}^{v} \\
& s_{(k)(j)}^{w}=\frac{w_{(j)}-w_{(k)}}{\xi}, t_{(k)(j)}^{w}=\frac{1}{2} f_{(k)(j)}^{w} \\
& s_{(k)(j)}^{\theta_{x}}=\frac{\theta_{x(j)}-\theta_{x(k)}}{\xi}, t_{(k)(j)}^{\theta_{x}}=\frac{1}{2} f_{(k)(j)}^{\theta_{x}} \\
& s_{(k)(j)}^{\theta_{y}}=\frac{\theta_{y(j)}-\theta_{y(k)}}{\xi}, t_{(k)(j)}^{\theta_{y}}=\frac{1}{2} f_{(k)(j)}^{\theta_{y}} \\
& s_{(k)(j)}^{\theta_{z}}=\frac{\theta_{z(j)}-\theta_{z(k)}}{\xi}, t_{(k)(j)}^{\theta_{z}}=\frac{1}{2} f_{(k)(j)}^{\theta_{z}}
\end{aligned}
$$

with $f_{(k)(j)}^{u}, f_{(k)(j)}^{v}, f_{(k)(j)}^{w}, f_{(k)(j)}^{\theta_{x}}, f_{(k)(j)}^{\theta_{y}}, f_{(k)(j)}^{\theta_{z}}$ can be calculated by using Eq. (29).

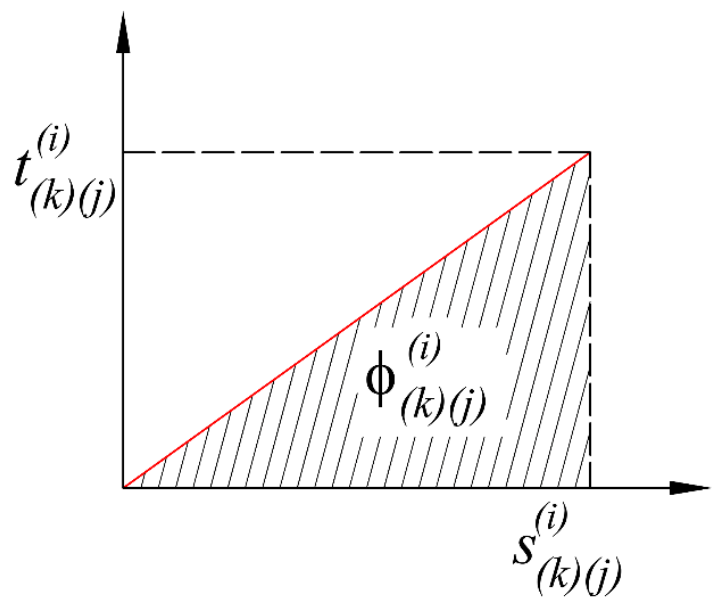

Fig. 5. A linear relationship between bond stretch and force density

\section{Numerical procedure}


The PD equation of motion can be solved by using a meshless scheme. The domain is divided into a uniform grid, with material points associated with specific volumes. The time integration can be achieved through an explicit scheme using the adaptive dynamic relaxation method described by Kilic and Madenci (2010) (See Appendix C). The numerical procedure is shown in Fig. 6. 


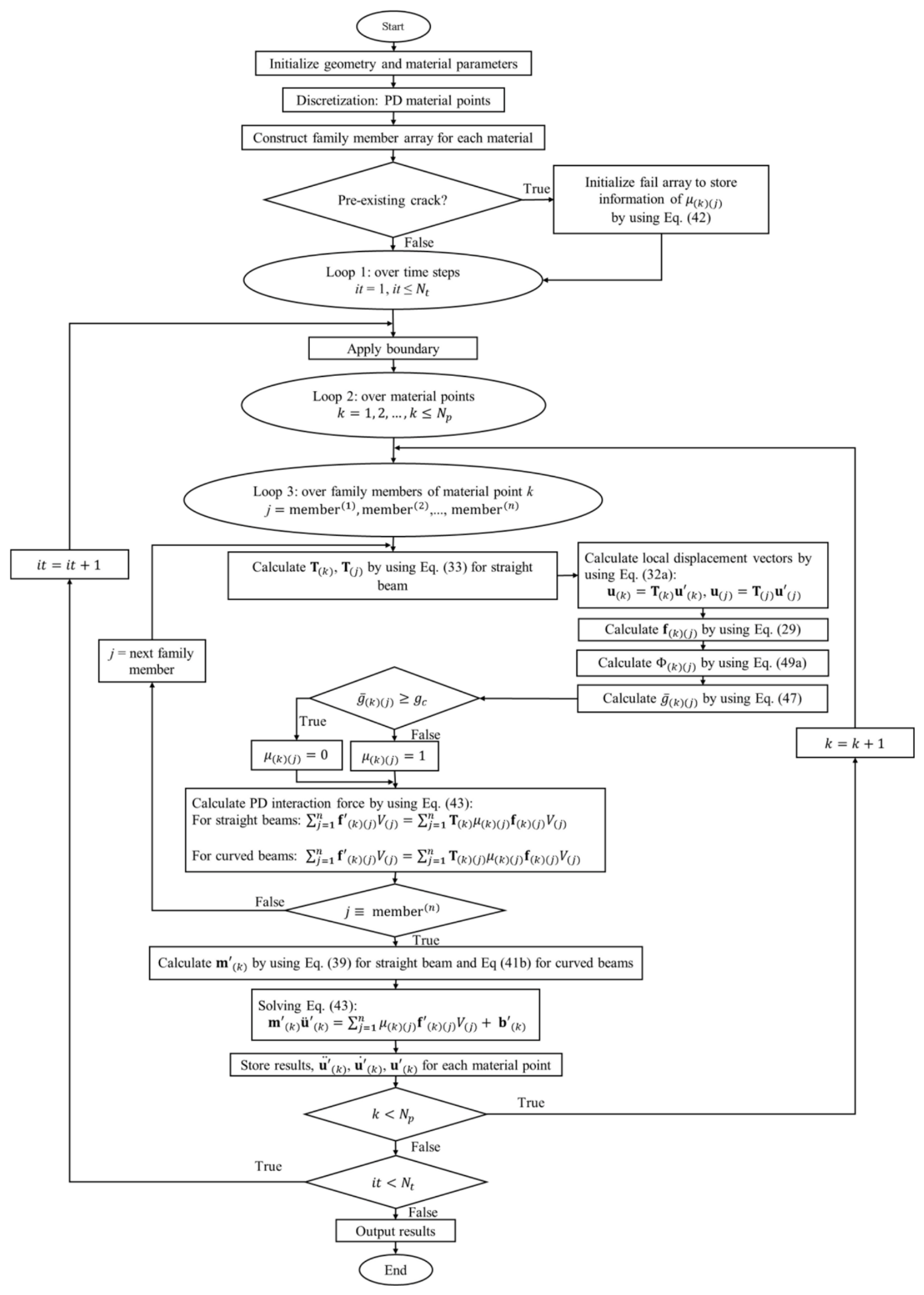

Fig. 6. Numerical procedure 


\subsection{Model discretization}

Fig. 7 demonstrates the model discretization for pin joined beam structures. As shown in Fig. 7, three beams A, B, and C are joined together at one point. In order to generate material points for this structure, each beam is firstly discretized into material points. After the discretization, for the points that share the same location such as points $i^{(A)}, k^{(B)}, j^{(C)}$ as shown in Fig. 7, only one material point is kept, the other points are removed. Point $k^{(A, B, C)}$ in Fig. 7 represents the joint point for three beams.

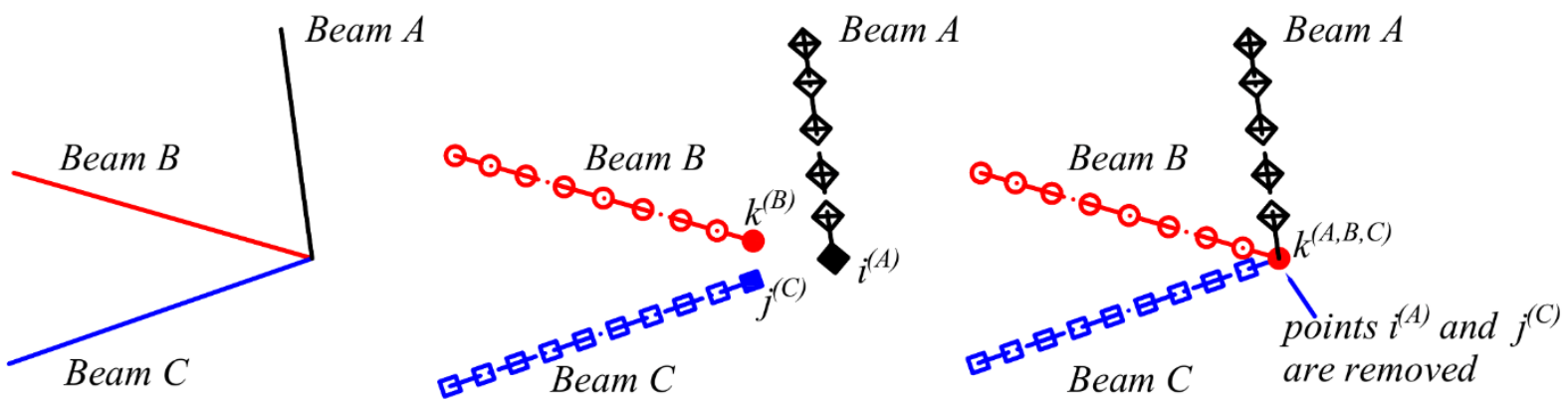

Fig. 7. The connection of beams at joint points

\subsection{Geometrical and material properties at the joint points}

It is considered that the family members for a joint point $k^{(A, B, C)}$ in Fig. 7 includes the material points from all three beams. If material point $k^{(A, B, C)}$ interacts with material points in beam $\mathrm{A}$, the material properties and geometrical parameters of material point $k^{(A, B, C)}$ are set same as beam A. On the other hand, if material point $k^{(A, B, C)}$ interacts with material points in beam $\mathrm{B}$, the material properties and geometrical parameters of material point $k^{(A, B, C)}$ are set same as beam B.

\section{Numerical results}

For verification purposes, the proposed PD model is first compared with finite element solutions. In section 7.1, the PD prediction for static problems are compared with ANSYS commercial software by using BEAM188 element. Next, in section 7.2, the PD prediction for a dynamic problem are compared with Sesam GeniE software provided by DNV GL. Sesam GeniE is a tool that is used for structural analyses of offshore and marine structures with environmental loading calculations including sea and wind loads.

The beams are made of steel material with Young's modulus $E=2 \times 10^{11} \mathrm{~N} / \mathrm{m}^{2}$, shear modulus $G=1 \times 10^{11} \mathrm{~N} / \mathrm{m}^{2}$, and mass density $\rho=7850 \mathrm{~kg} / \mathrm{m}^{2}$. For static solutions, an explicit scheme is used by implementing adaptive dynamic relaxation method (Kilic and Madenci, 2010), (Underwood, 1983).

\subsection{Static analyses}

This section presents static analyses of beam type structures in peridynamics. The PD results are verified by comparing with ANSYS solutions. The PD predictions for a 2D frame with straight beams, a curved beam and a jacket platform subjected to concentrated loads are presented. In all 
these examples, the weight of the structure is ignored.

\subsubsection{D frame with straight beams subjected to a constant concentrated load}

In order to verify the developed PD model for straight beams, a 2D frame subjected to point load is investigated. The steel frame consists of two beams as shown in Fig. 8. The frame is subjected to a concentrated force $F_{z^{\prime}}=-3 \times 10^{6} \mathrm{~N}$ at $(L, L, 0)$ and it is clamped at both ends. Both beams have the same length $L=1 \mathrm{~m}$ and same square cross-section, $A=0.1 \times 0.1 \mathrm{~m}^{2}$.

In the peridynamic model, both beams are discretized with uniform 200 integration points. In order to implement the fixed ends of the frame, three fictitious points (Oterkus and Madenci, 2015, 2017) are added at both ends and all the displacement and rotation components of these fictitious points are set equal to zero. In Fig. 8b, red points represent the material points in the real region, on the other hand, black points represent the material points in the fictitious region. In the FEA model, each beam is meshed with 200 elements.

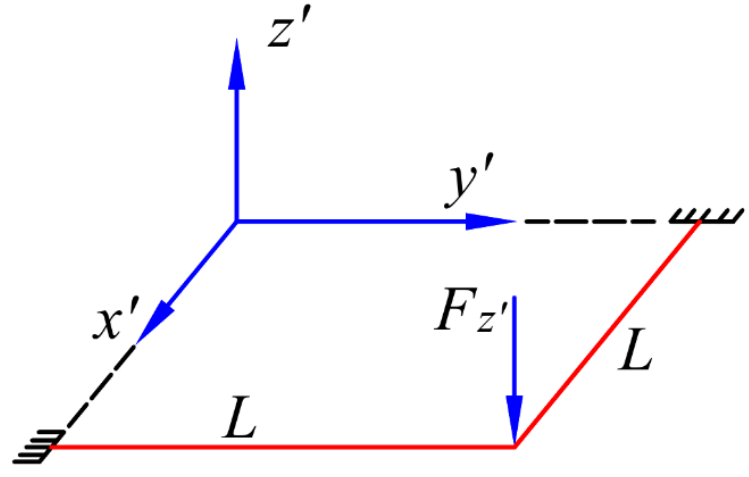

(a)

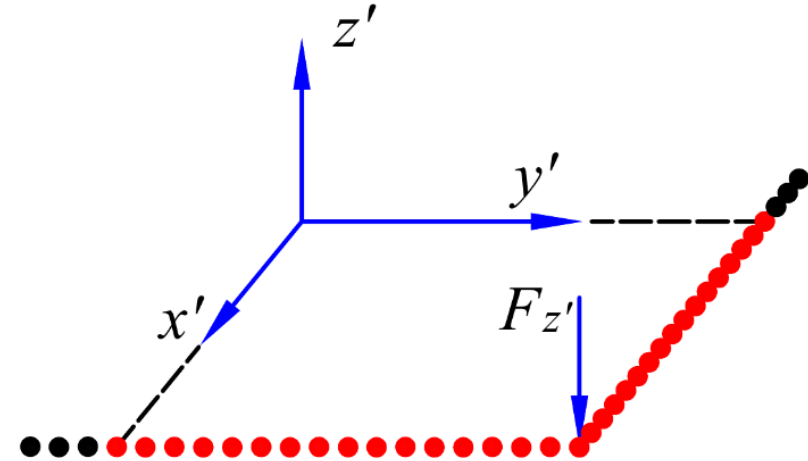

(b)

Fig. 8. 2D frame subjected to concentrated load (a) geometry, (b) PD discretization Due to the small deformation assumption, the beams have three nonzero DOFs. These are transverse displacement $w^{\prime}$, rotation $\theta_{x^{\prime}}$ and $\theta_{y^{\prime}}$. Figs. 9-11 represent the displacement variations along each beam in the deformed configuration. As can be seen the figures, PD results agree very well with the FEA results which shows the accuracy of the developed PD model for straight beams.

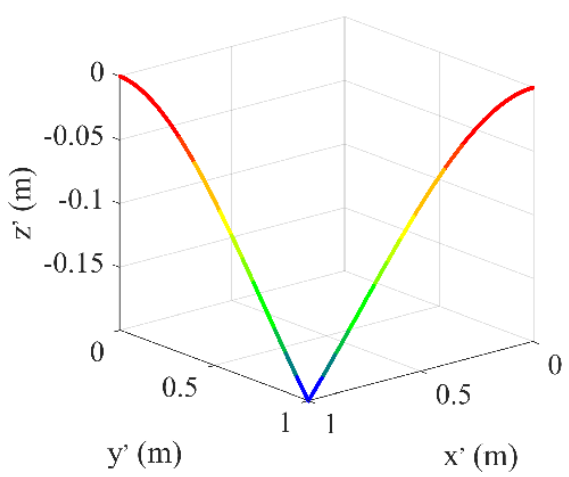

(a)

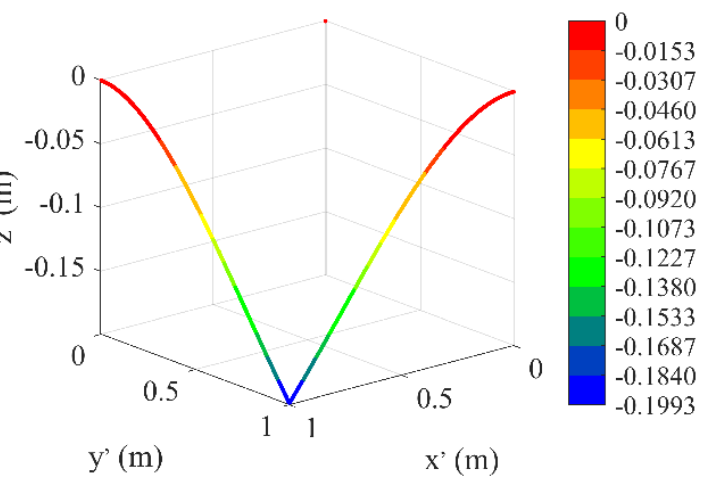

(b)

Fig. 9. Variation of displacement $w^{\prime}$ in deformed configuration (a) PD, (b) FEA 


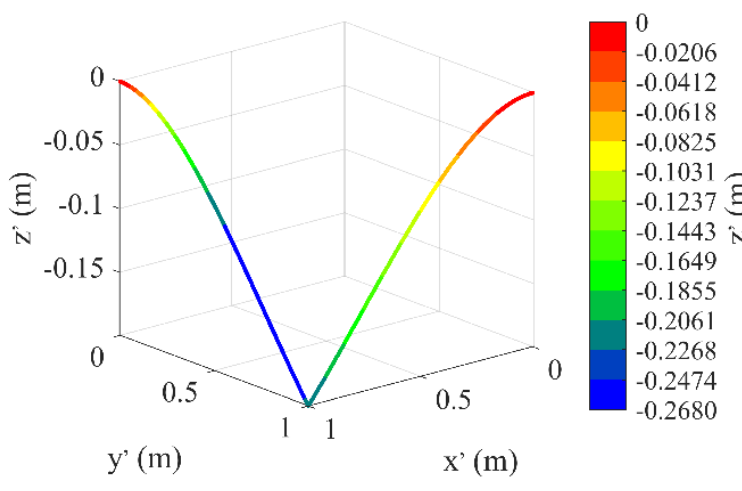

(a)

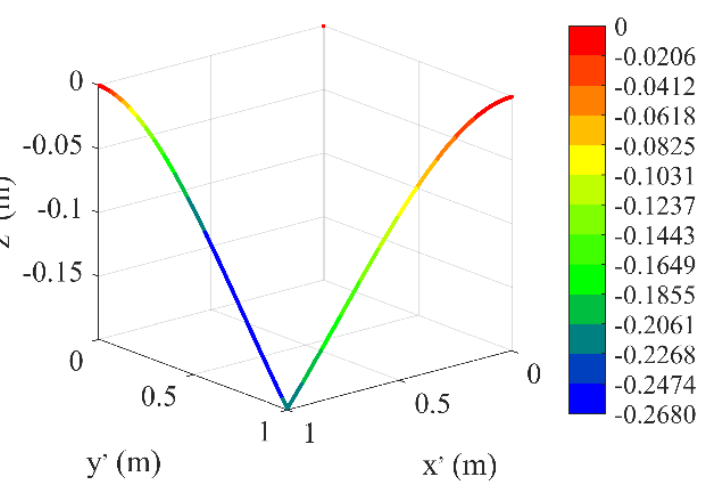

(b)

Fig. 10. Variation of $\theta_{x^{\prime}}$ in deformed configuration (a) PD, (b) FEA

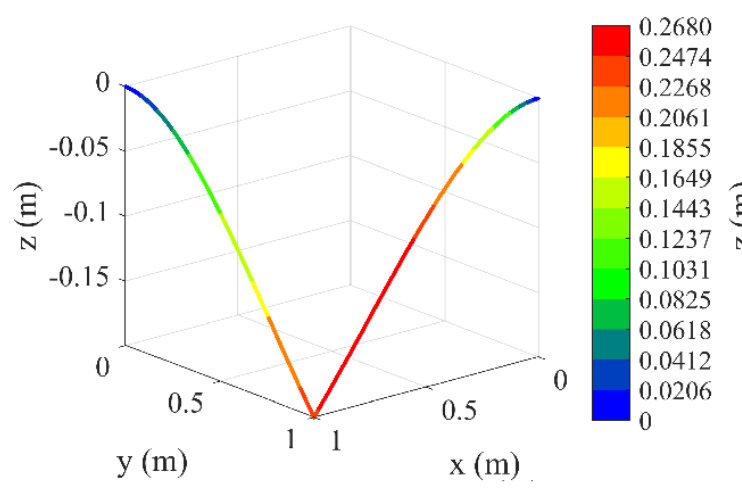

(a)

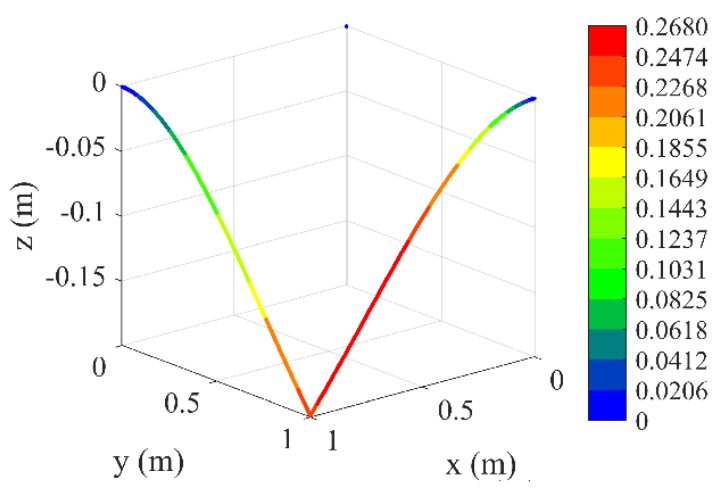

(b)

Fig. 11. Variation of $\theta_{y^{\prime}}$ in deformed configuration (a) PD, (b) FEA

\subsubsection{Curved beam subjected to a constant concentrated load}

In order to verify the developed PD model for curved beams, a semi-circular beam of radius $R$ subjected to point load is investigated as shown in Fig.12. The semi-circular beam is clamped at both ends and it is subjected to static loading $F_{y^{\prime}}=-1 \times 10^{8} \mathrm{~N}$ and $F_{z^{\prime}}=-2 \times 10^{6} \mathrm{~N}$. The semicircular beam has a radius of $R=0.5 \mathrm{~m}$ and square cross-section as $b=h=0.1 \mathrm{~m}$. 


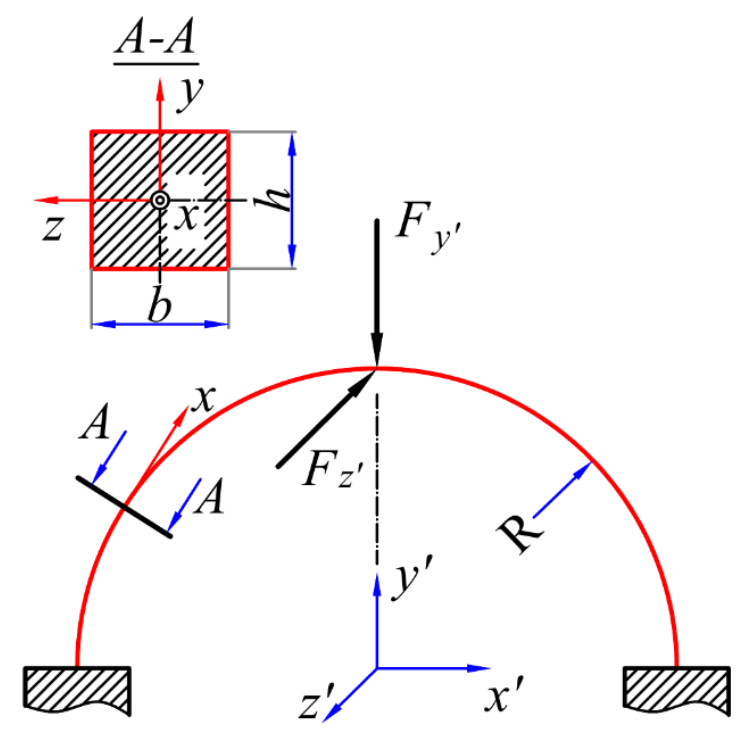

(a)

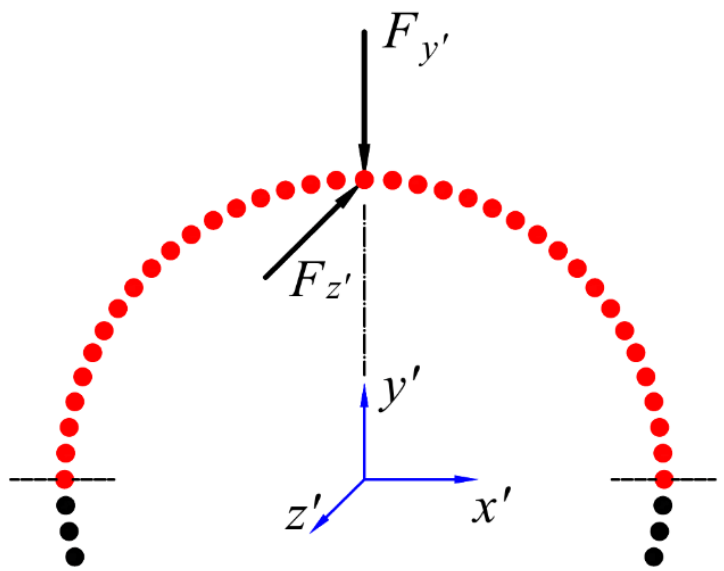

(b)

Fig. 12. Semi-circular beam subjects to concentrated load (a) geometry, (b) PD discretization

In the peridynamic model, the beam is discretized with uniform 500 integration points. In the FEA model, the beam is meshed with 500 elements. In order to implement the fixed ends of the frame, three fictitious points are added at both ends and all the displacement and rotation components of these fictitious points are set equal to zero. As shown in Fig. 12b, red points represent the material points in the real region, and black points represent the material points in the fictitious region.

Figs.13-14 represent the deformed shape of the beam and displacement and rotation variations along the semi-circular beam, respectively. It can be seen from Fig. 13 that the deformed shape of the beam predicted by PD agrees very well with the FEA solutions. It is also observed that all 6 DOFs of the beam predicted by PD and FEA have a very good agreement as shown in Fig. 14.

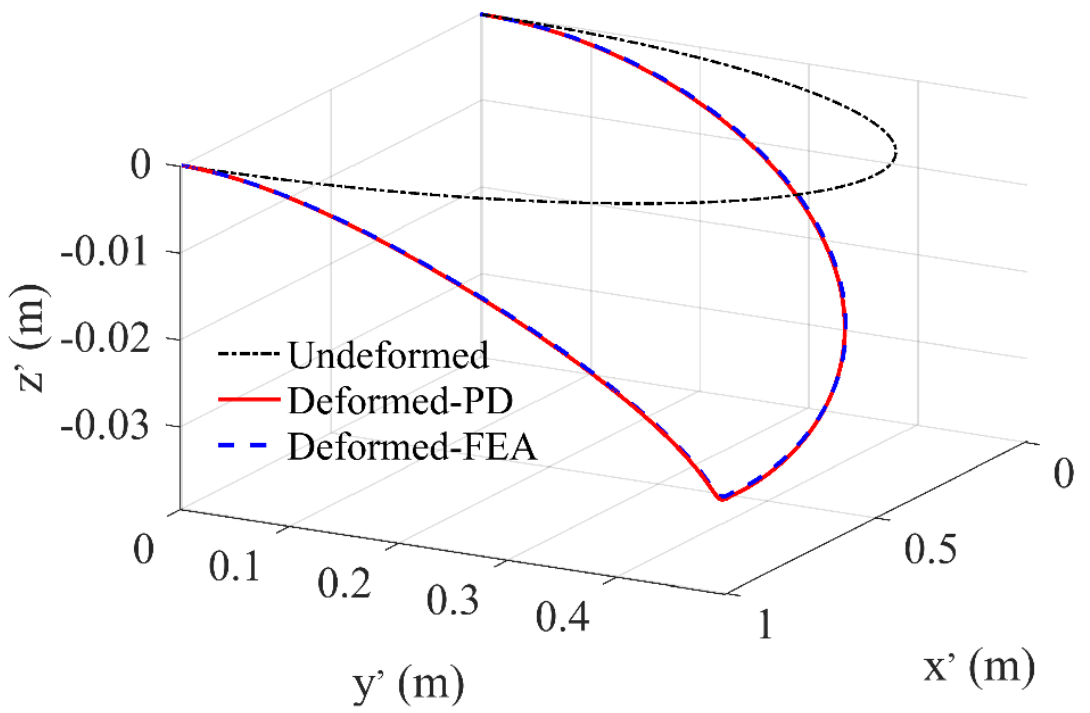

Fig. 13. The deformed and un-deformed shape of the beam 


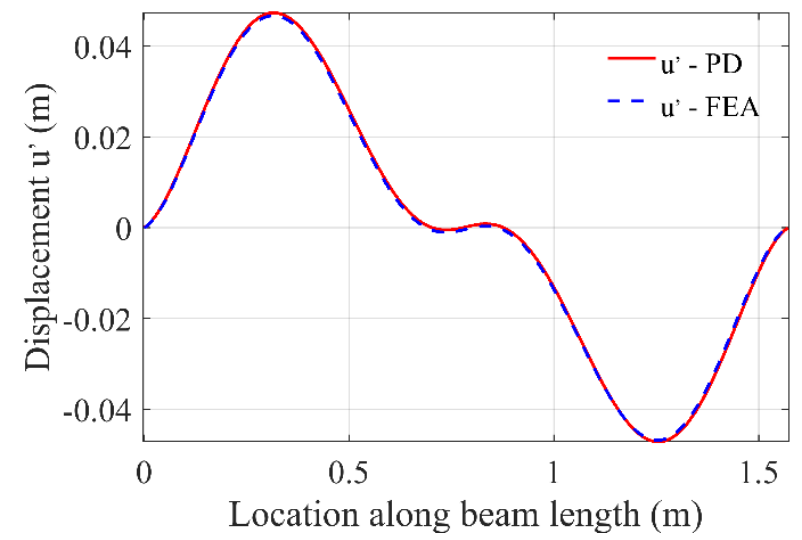

(a)

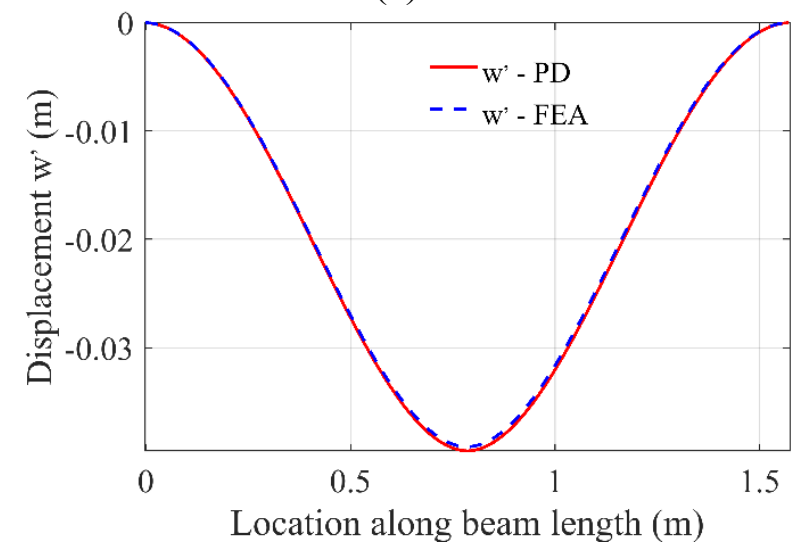

(c)

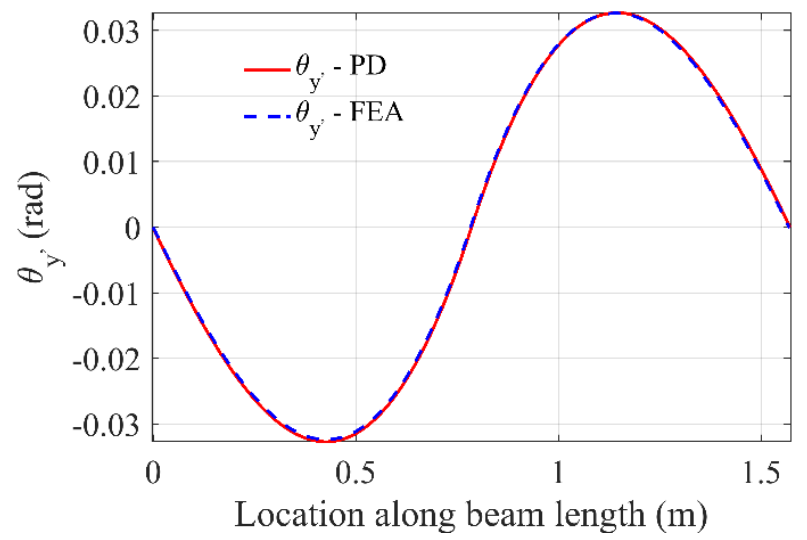

(e)

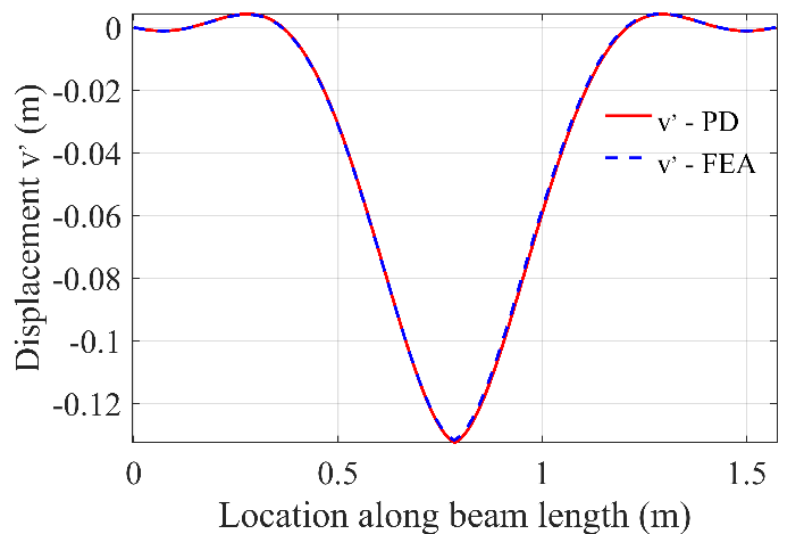

(b)

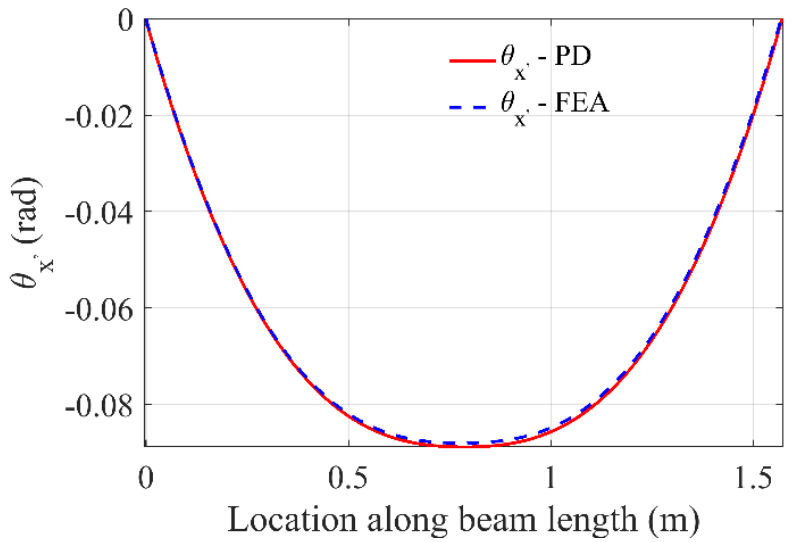

(d)

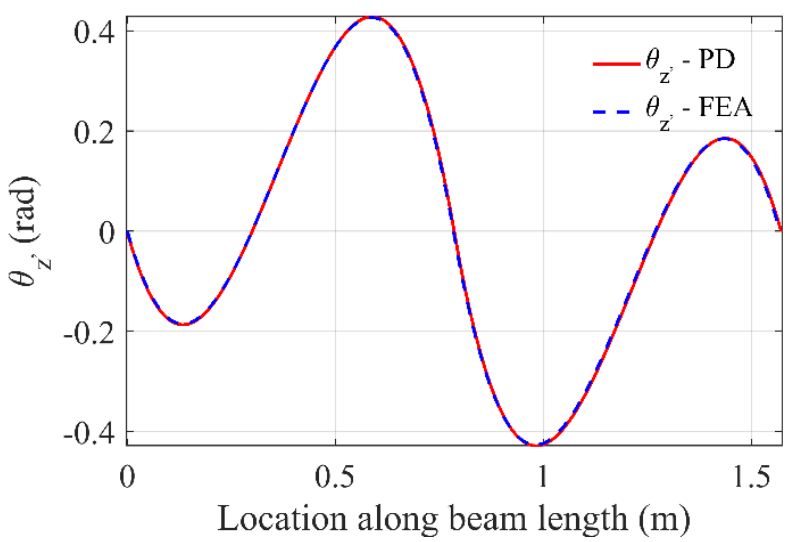

(f)

Fig. 14. Variation of (a) $u^{\prime}$, (b) $v^{\prime}$, (c) $w^{\prime}$, (d) $\theta_{x^{\prime}}$, (e) $\theta_{y^{\prime}}$, (f) $\theta_{z^{\prime}}$ along the beam length

\subsubsection{Jacket platform subjected to a constant concentrated load}

After verifying the developed PD model for the simple frame and curved beam, the accuracy of the PD model is tested for a jacket platform which consists of 28 beam type structures and 16 joints. For this purpose, a $27 \mathrm{~m}$ high jacket platform is modeled as shown in Fig. 15. The jacket platform has the following dimensions; $z_{1}^{\prime}=9 \mathrm{~m}, z_{2}^{\prime}=18 \mathrm{~m}, z_{3}^{\prime}=27 \mathrm{~m}, L_{1}=L_{2}=12 \mathrm{~m}$, $L_{3}=L_{4}=4 \mathrm{~m}$. The jacket platform is fixed on 4 legs at the location of $z^{\prime}=0$ and subjected to 
concentrated loading $F_{y^{\prime}}=2 \times 10^{7} \mathrm{~N}$ at $\left(L_{3} / 2,-L_{4} / 2, z_{3}^{\prime}\right)$ and $\left(-L_{3} / 2,-L_{4} / 2, z_{3}^{\prime}\right)$. Each beam component of the jacket platform has a hollow circular cross-section. Four main legs, shown in red, have an outer diameter $0.6 \mathrm{~m}$ and inner diameter $0.576 \mathrm{~m}$, the other beams, shown in blue, have an outer diameter $0.4 \mathrm{~m}$, and the inner diameter $0.384 \mathrm{~m}$.

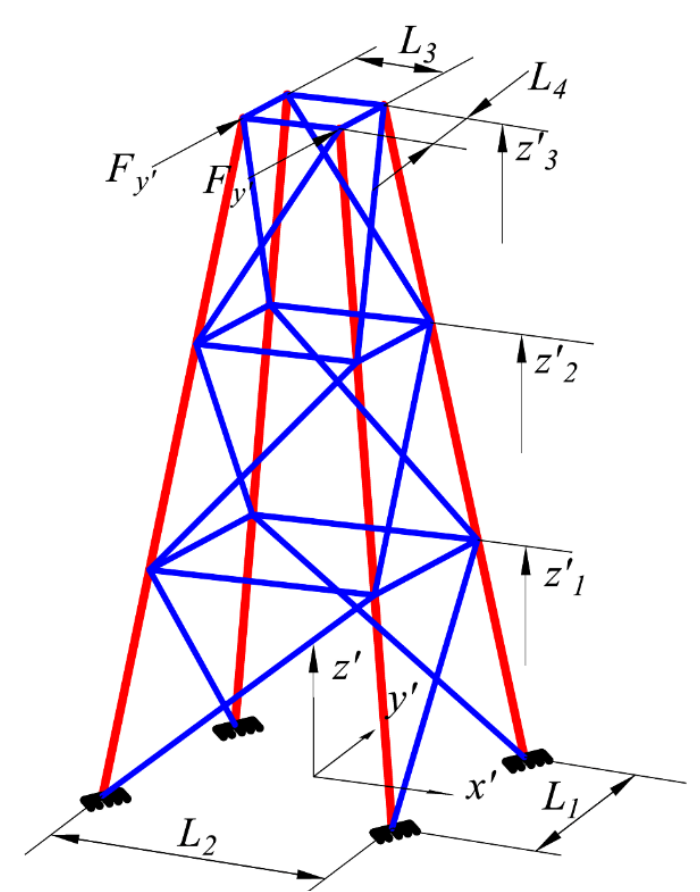

(a)

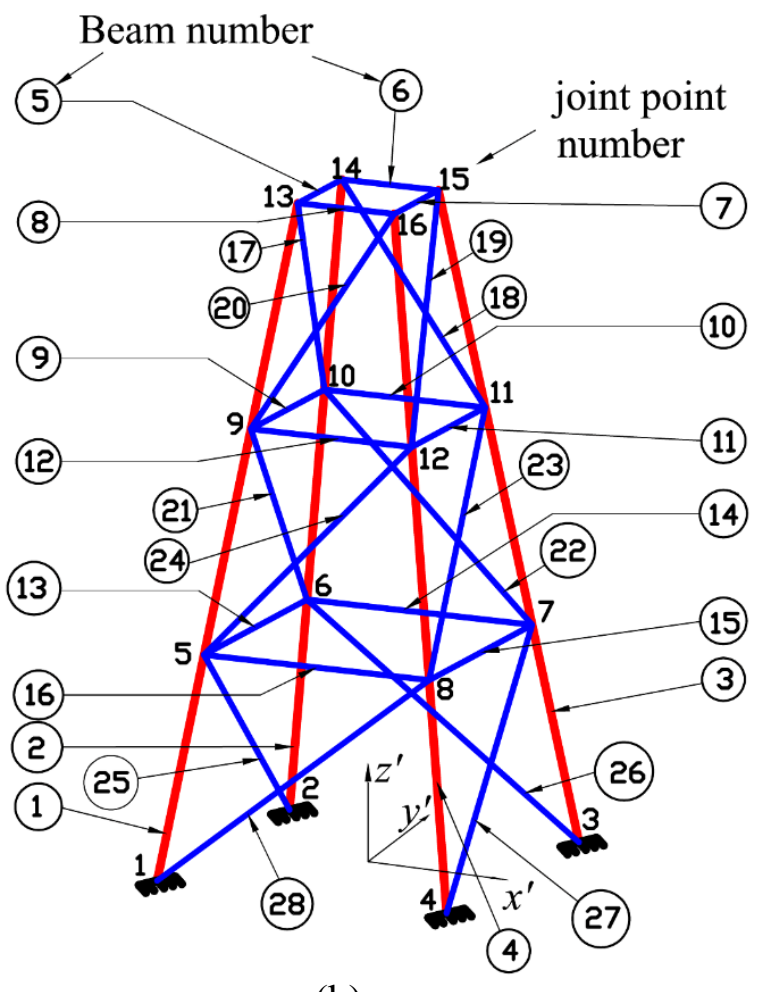

(b)

Fig. 15. Jacket platform (a) geometry, (b) beam numbers and joint points

In the PD model, beam 1, 2, 3, 4 (shown in red) are discretized into 300 material points, and other beams (shown in blue) are discretized into 100 material points. Similarly, for the ANSYS model, beam 1, 2, 3, 4 are meshed with 300 elements, and other beams are meshed with 100 elements.

Similar to the previous examples, in order to apply boundary conditions, three fictitious material points are added for beam 1,2, 3, 4, 25, 26, 27, and 28 on the center line of each beam, a long negative $z^{\prime}$ direction, and all DOF of these fictitious points are set equal to zero.

Shown in Figs. 16-21 is the variation of 6 DOFs of the jacket platform in the deformed shape in the global coordinates. The results obtained from the PD analysis match very well with those in FEA. 


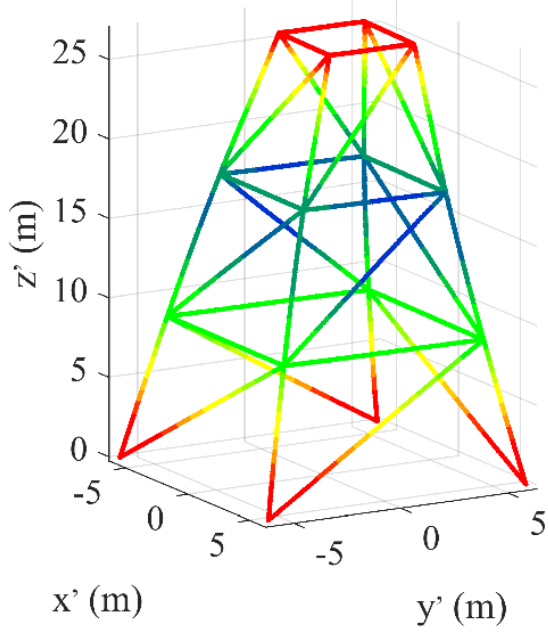

(a)

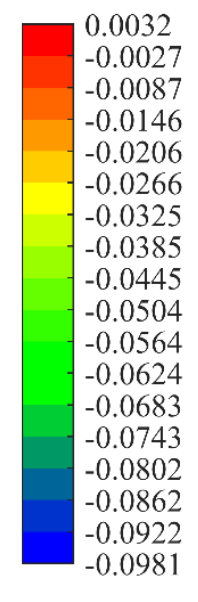

(2)

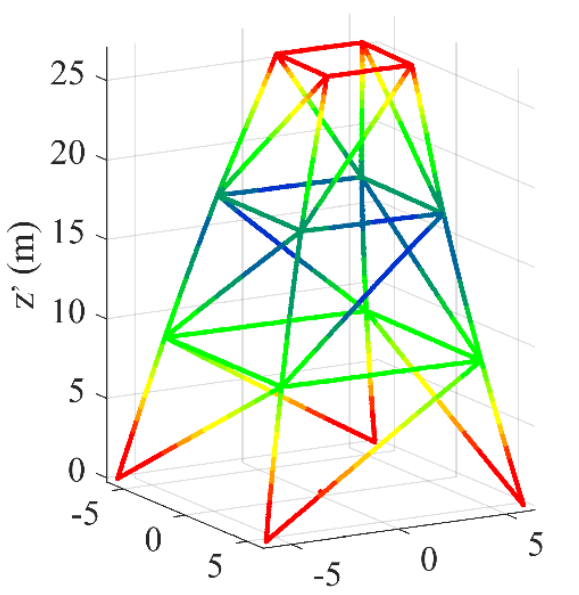

$y^{\prime}(m)$

(b)

Fig. 16. Displacement $u^{\prime}$ in deformed configuration (a): PD analysis, (b): FEA

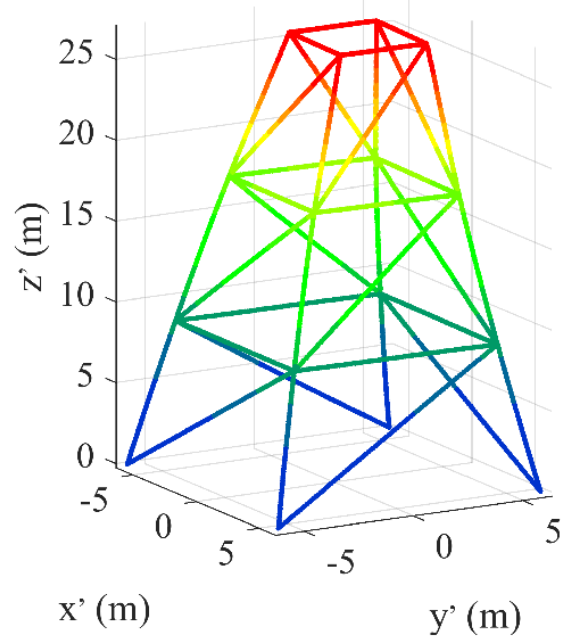

(a)
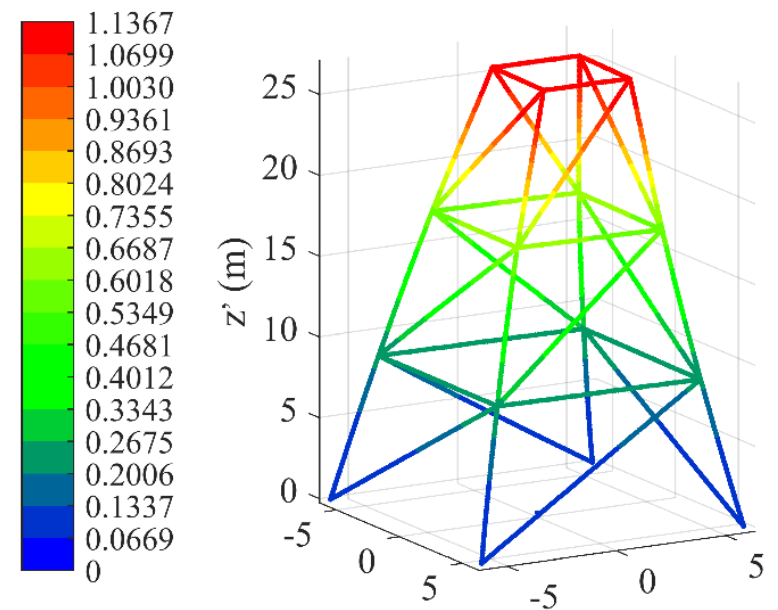

$y^{\prime}(m)$

(b)

Fig. 17. Displacement $v^{\prime}$ in deformed configuration (a): PD analysis, (b): FEA 


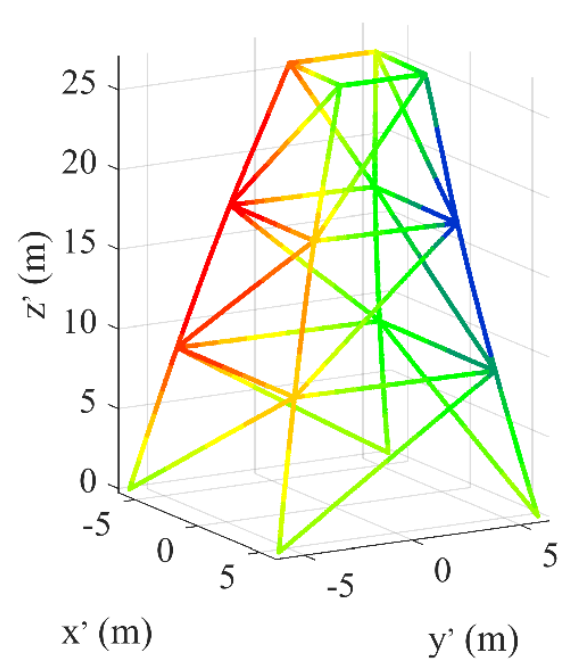

(a)

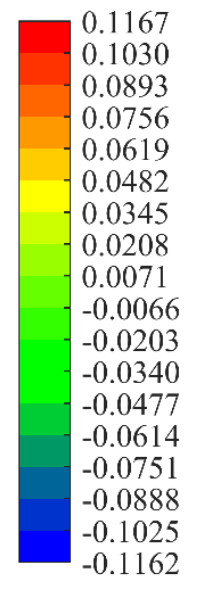
0.1030 0.0893

0.0482

.0345

.0071

$-0.0340$

$-0.075$

$-0.0888$

0.1162

$y^{\prime}(m)$

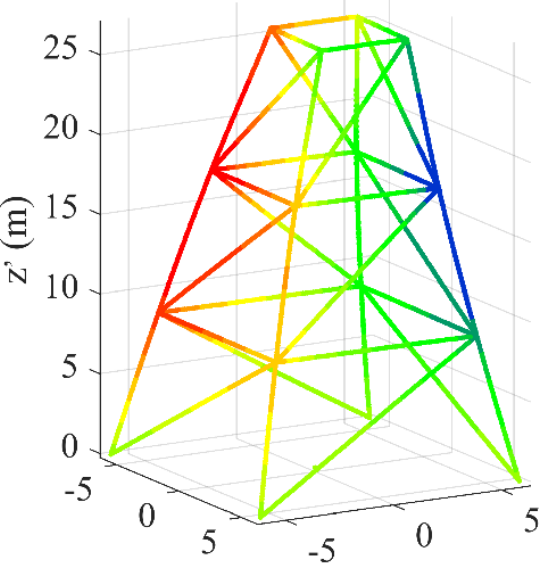

0.1167

0.1030

0.0893

0.0756

0.0619

0.0482

0.0345

0.0208

0.0071

$-0.0066$

$-0.0203$

$-0.0340$

$-0.0477$

$-0.0614$

$-0.0751$

$-0.0888$

$-0.1025$

$-0.1162$

$\mathrm{x}^{\prime}(\mathrm{m})$

$y^{\prime}(m)$

(b)

Fig. 18. Displacement $w^{\prime}$ in deformed configuration (a): PD analysis, (b): FEA

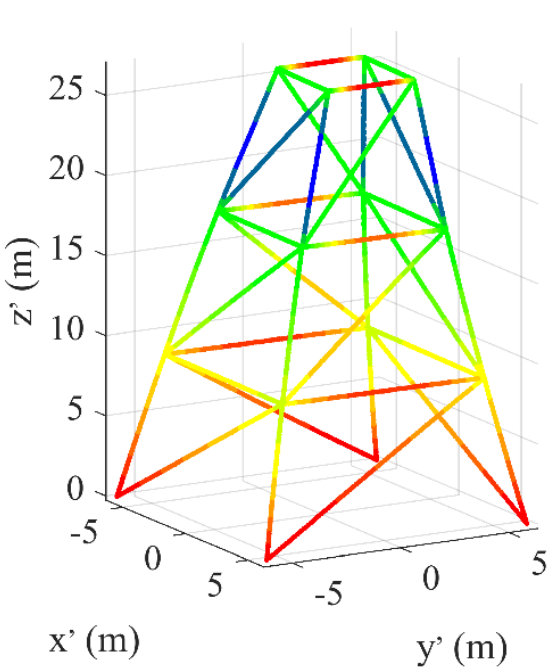

(a)
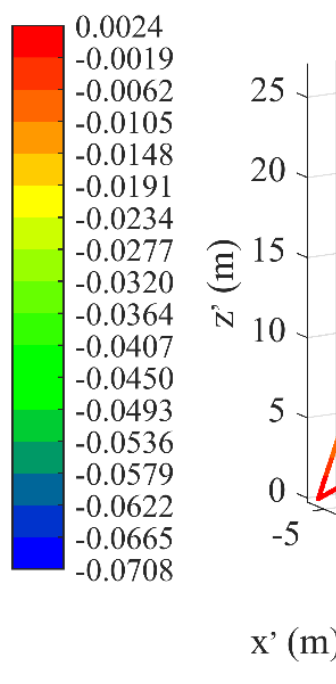

0.0024

$-0.0019$

$-0.0062$

$-0.0105$

$-0.0148$

$-0.0191$

$-0.0234$

$-0.0277$

$-0.0320$

$-0.0364$

$-0.0407$

$-0.0450$

$-0.0493$

$-0.0536$

$-0.0579$

$-0.0622$

$-0.0665$

$-0.0708$

$y^{\prime}(m)$

(b)

Fig. 19. Rotational angle $\theta_{x^{\prime}}$ in deformed configuration (a): PD analysis, (b): FEA 


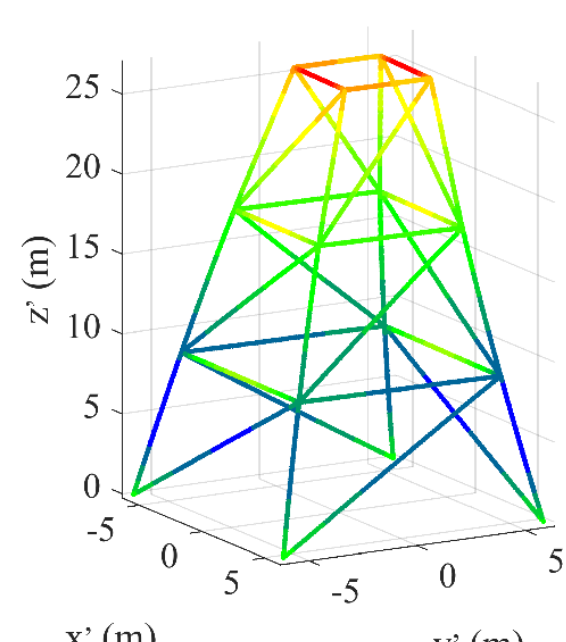

$\mathrm{x}^{\prime}(\mathrm{m})$

(a)

$\mathrm{y}^{\prime}(\mathrm{m})$

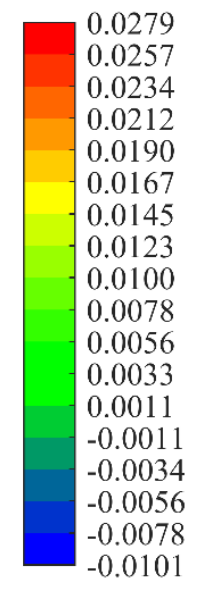

Fig. 20. Rotational angle $\theta_{y^{\prime}}$ in deformed configuration (a): PD analysis, (b): FEA

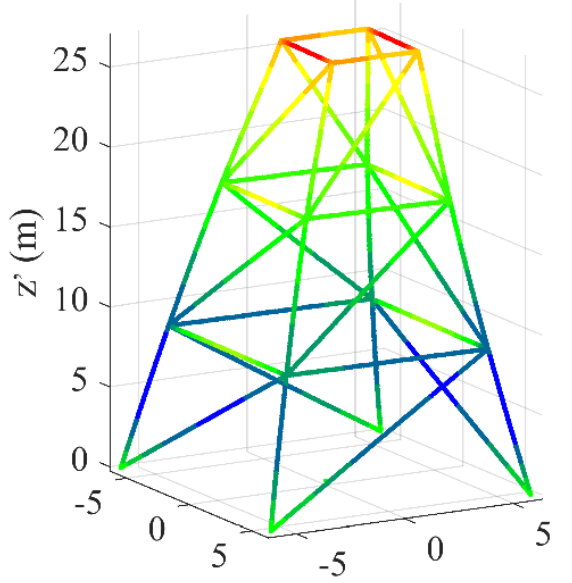

$\mathrm{X}^{\prime}(\mathrm{m})$

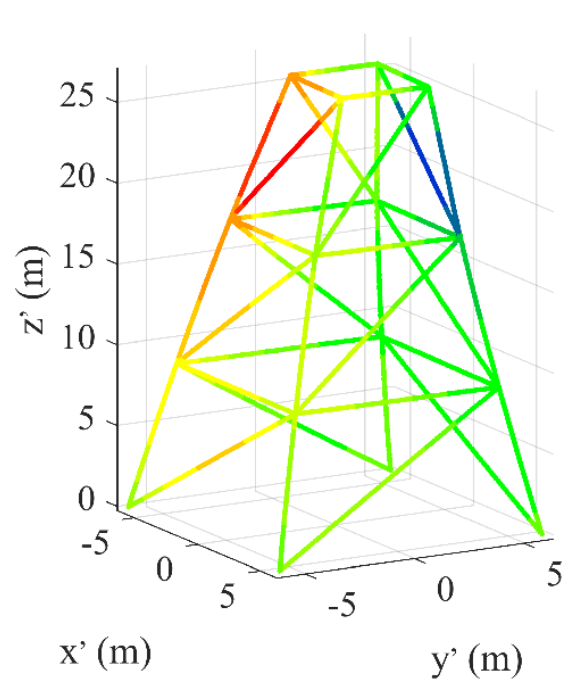

(a)

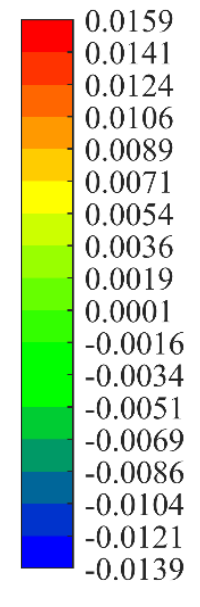

$\mathrm{y}^{\prime}(\mathrm{m})$

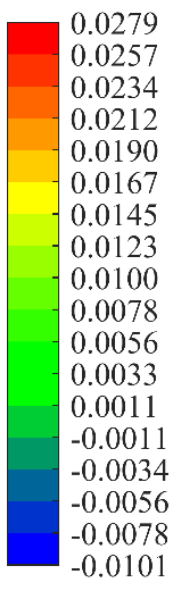

$y^{\prime}(m)$

(b)
.0279

0212

0190

.0123

0.0100

0.0056

0.0033

0.0011

0.0056

$-0.0101$ 

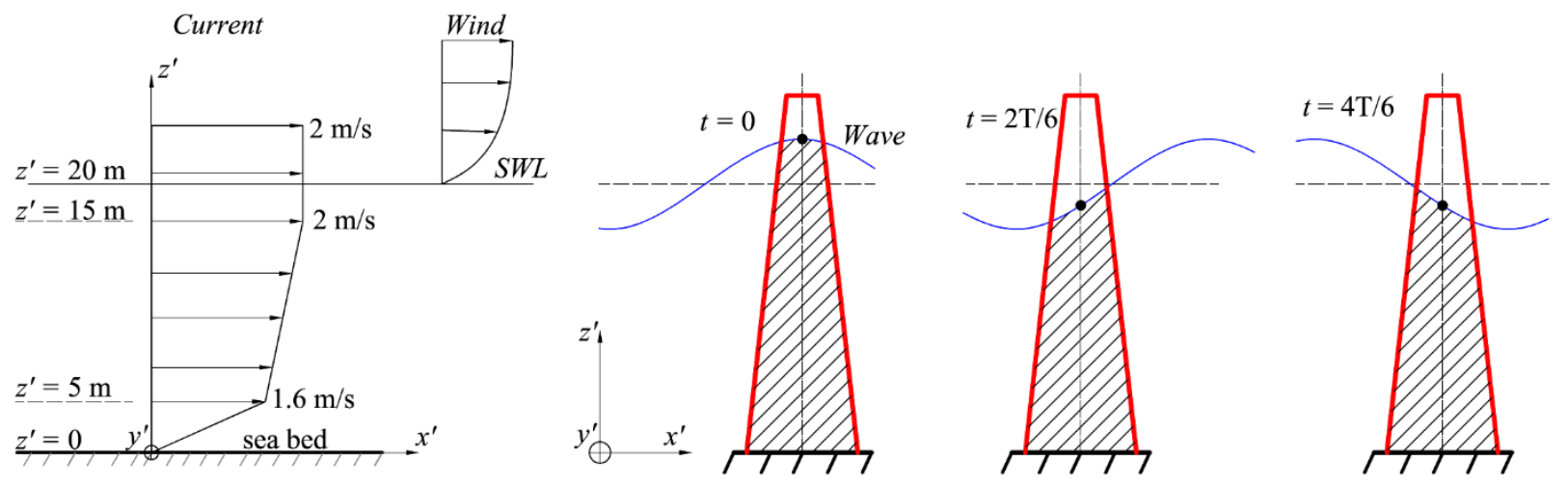

Fig. 22. Jacket platform is subjected to sea load

As shown in Fig. 22, the jacket platform is subjected to sea environment with water depth $d=20 \mathrm{~m}$, wave height $H=10 \mathrm{~m}$, and wave period $T=30 \mathrm{~s}$. The environment is assumed as shallow water (Andersen et al., 2014) and the corresponding wavelength is calculated from Eq. (A.10) as $L=420 \mathrm{~m}$ which results in $d / L=0.0476$. Since $d / L<0.05$, shallow water assumption is validated.

The wind mean velocity is considered as $V_{(\text {wind })}=25 \mathrm{~m} / \mathrm{s}$ at a reference height $H_{0}=10 \mathrm{~m}$, the gust factor $f=1.5$, and the height exponent $P=0.16$ (see appendix B4 for descriptions of these parameters). The current profile is described in Fig. 22. The wind, wave, and current are assumed to be in the $x^{\prime}$ direction. In addition, the jacket is subjected to a loading $G_{0}=20 \times 10^{5} \mathrm{~N}$ in the negative $z^{\prime}$ direction which represents the weight of the superstructure as shown in Fig. 23. The wind load on superstructure is calculated by simplifying the superstructure as a rigid cube with the dimensions of $10 \mathrm{~m} \times 10 \mathrm{~m} \times 10 \mathrm{~m}$ in $x^{\prime}, y^{\prime}$, and $z^{\prime}$ directions, respectively.

The underwater part of each beam is applied loads induced by wave, current, and buoyancy. The above water part of each beam is subjected to wind force. The weight of the structure is also included as described in Appendix B1. The wind load acting on the platform is calculated by using Morison's equation described in Appendix B4. For the wind load calculation, it is assumed that only left part of the superstructure is exposed to wind load. The distributed wind load is calculated by assuming uniform mesh on the superstructure. Next, the wind force, $\mathbf{f}_{(\text {wind })(i)}$ and the corresponding location, $h_{\text {wind }(i)}$ of each element with respect to the top part of the jacket platform is calculated. Thus, the total wind force, $F_{\text {wind }}$ and its location $h_{\text {wind }}$, which are shown in Fig. 23, is obtained as

$$
\begin{aligned}
\mathbf{F}_{\text {wind }} & =\sum_{i=1}^{N_{s}} \mathbf{f}_{(\text {wind })(i)} \\
h_{\text {wind }} & =\frac{\sum_{i=1}^{N_{s}} h_{\text {wind }(i)} \mathbf{f}_{(\text {wind })(i)}}{\sum_{i=1}^{N_{s}} \mathbf{f}_{(\text {wind })(i)}}
\end{aligned}
$$


where $N_{s}$ represents the total number of mesh on the superstructure. The weight and wind load acting on the jacket platform due to superstructure are distributed to four joints of the jacket platform as shown in Fig. 23.

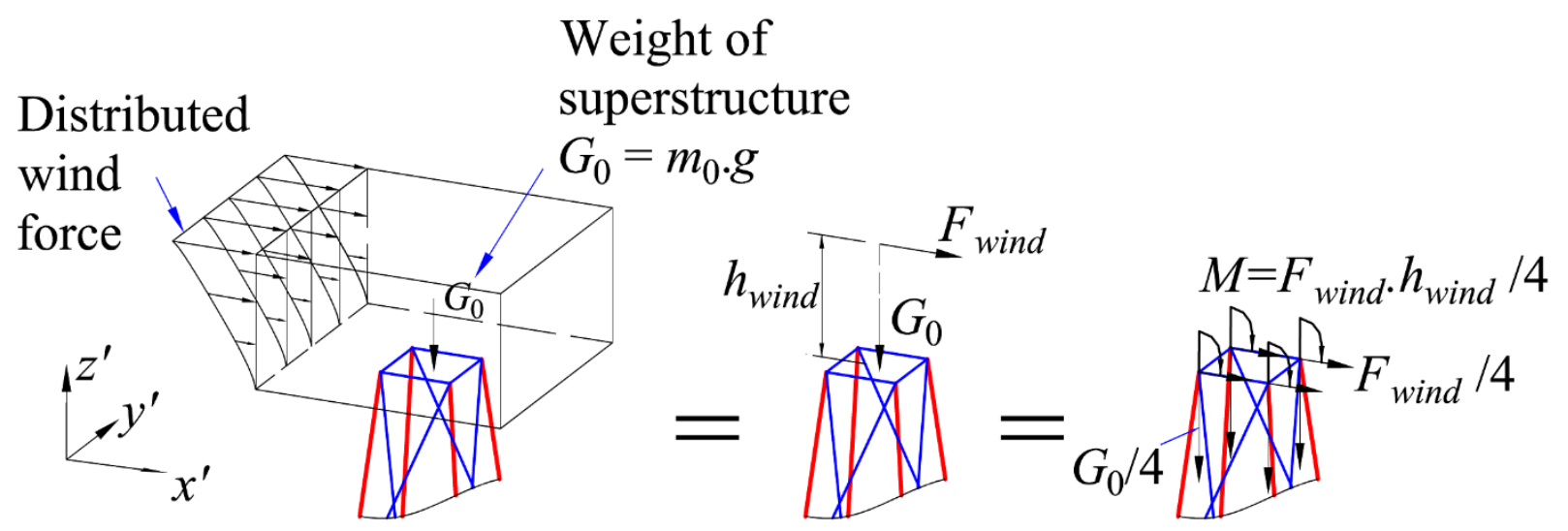

Fig. 23. Weight and wind load acting on the jacket platform due to the superstructure

Figs. 24-29 represent the variation of displacements and rotations of the jacket platform at different times. It can be seen that PD analysis and FEA using Sesam GeniE show a very good agreement of all 6 DOFs at all considered time points.

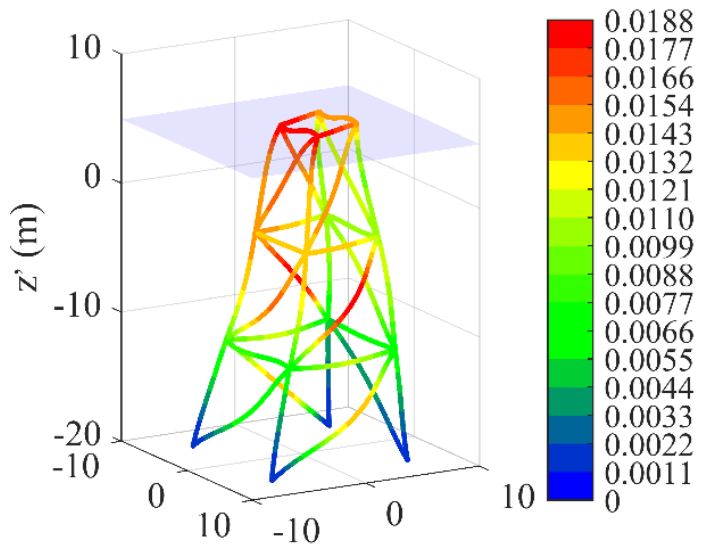

$\mathrm{X}^{\prime}(\mathrm{m})$

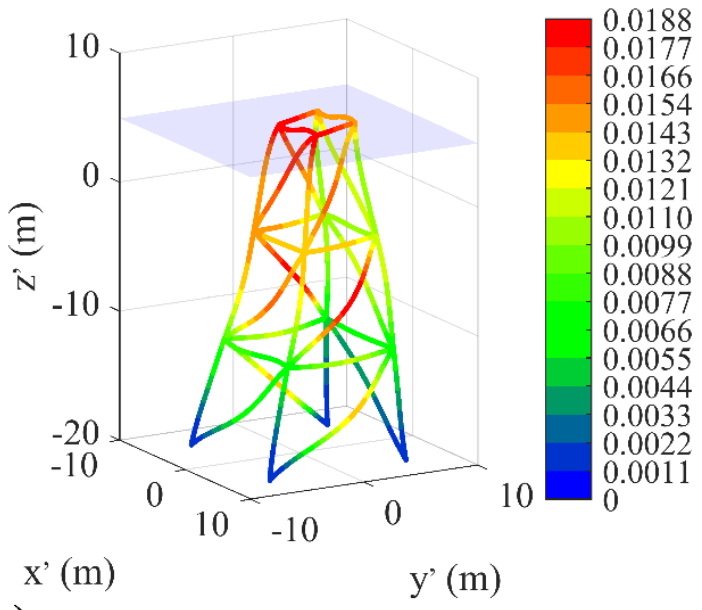

(a) 

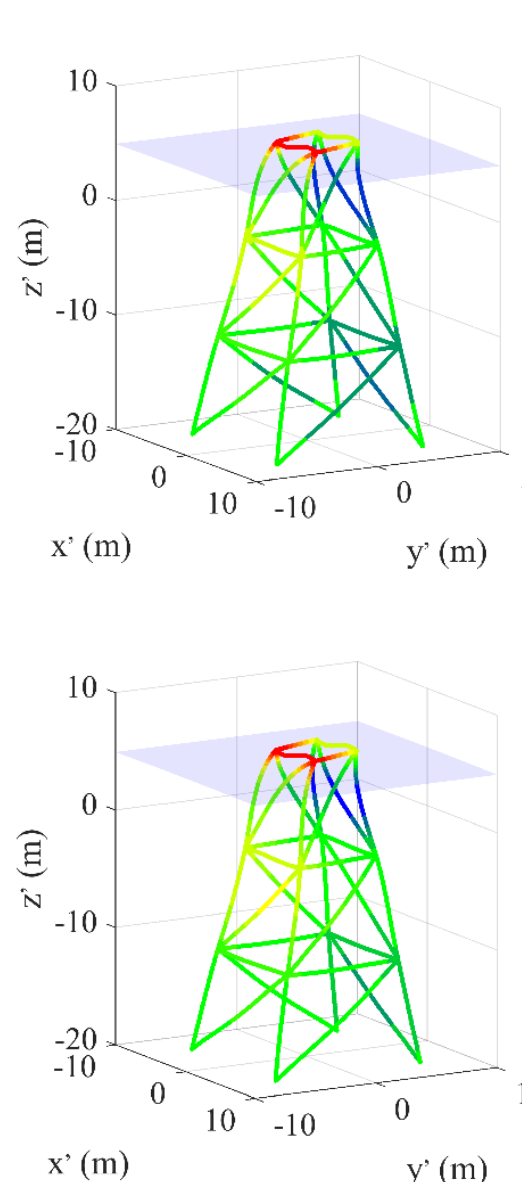

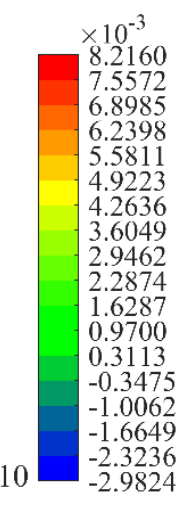

(b)

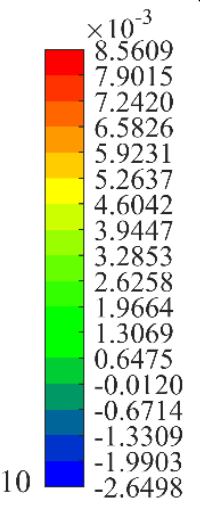

$y^{\prime \prime}(\mathrm{m})$

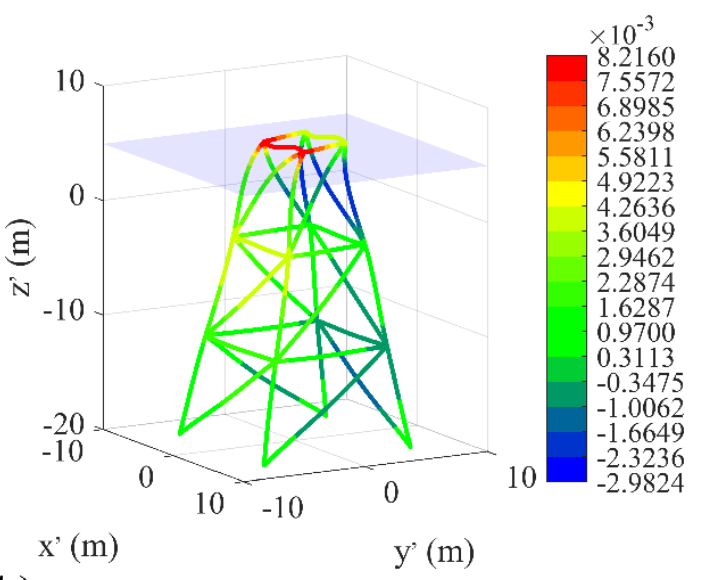

(b)

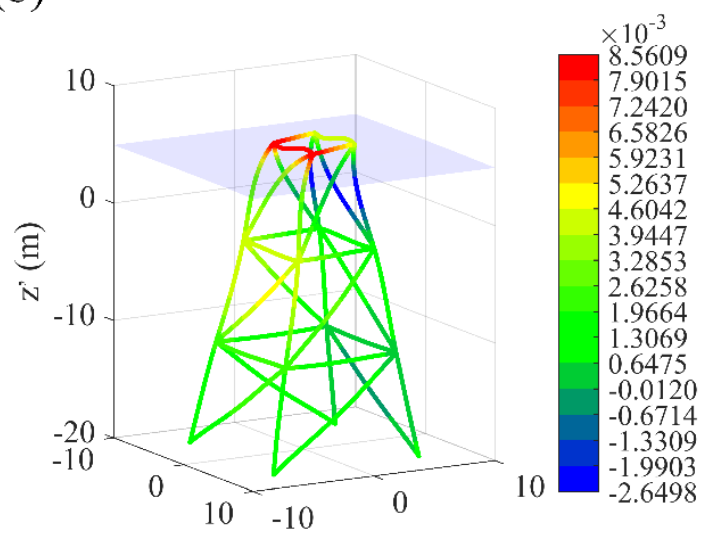

$\mathrm{x}^{\prime}(\mathrm{m}) \quad \mathrm{y}^{\prime}(\mathrm{m})$

(c)

Fig. 24. Variation of displacement $u^{\prime}$ in PD (left), Sesam GeniE (right) at (a) $t=0$, (b) $t=2 T / 6$, and (c) $t=4 T / 6$ (displacements are magnified by 200 for the deformed configurations)
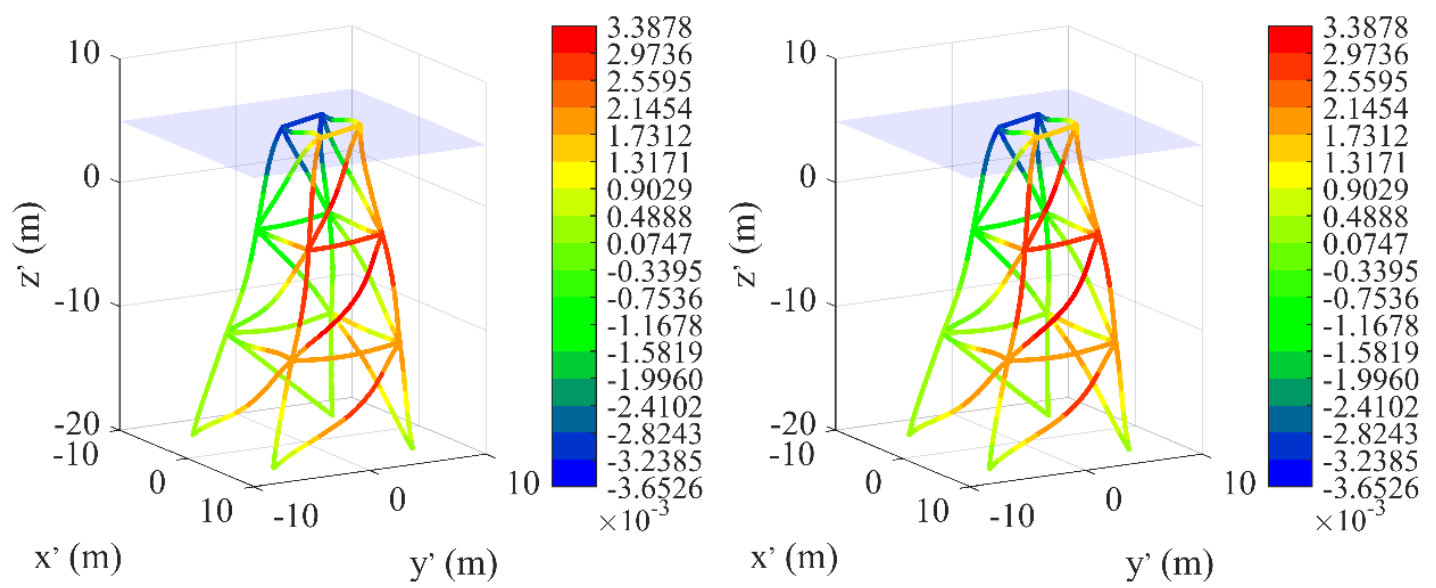

(a) 

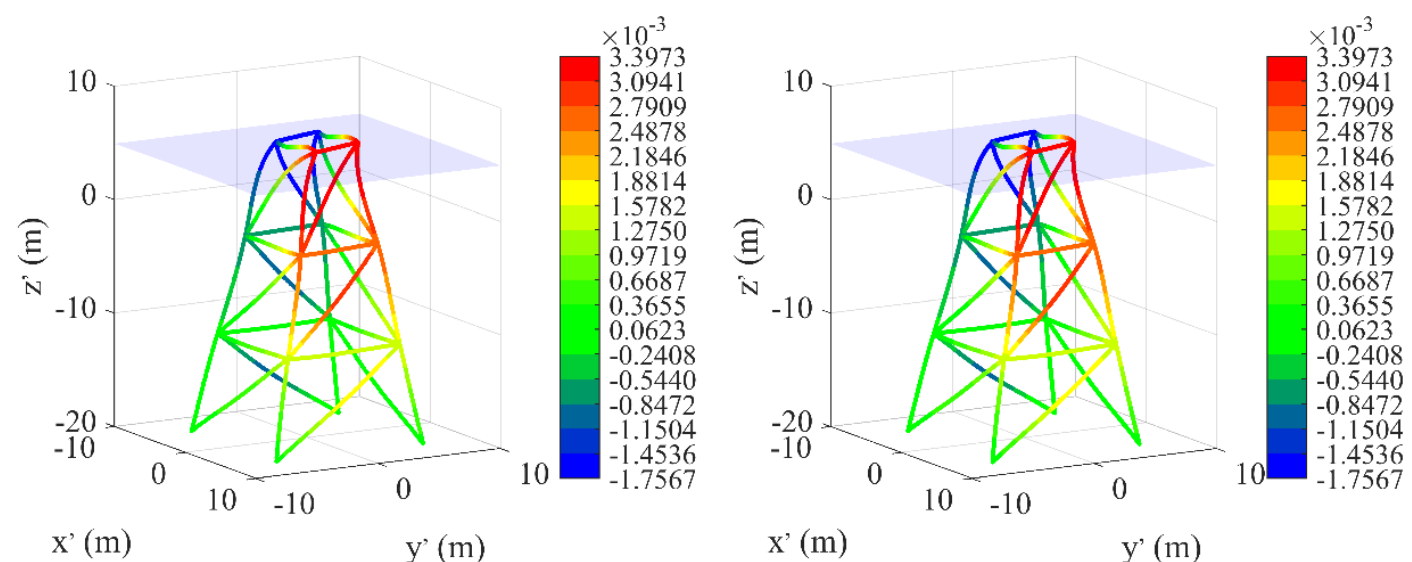

$\mathrm{x}^{\prime}(\mathrm{m})$

$y^{\prime}(m)$

(b)
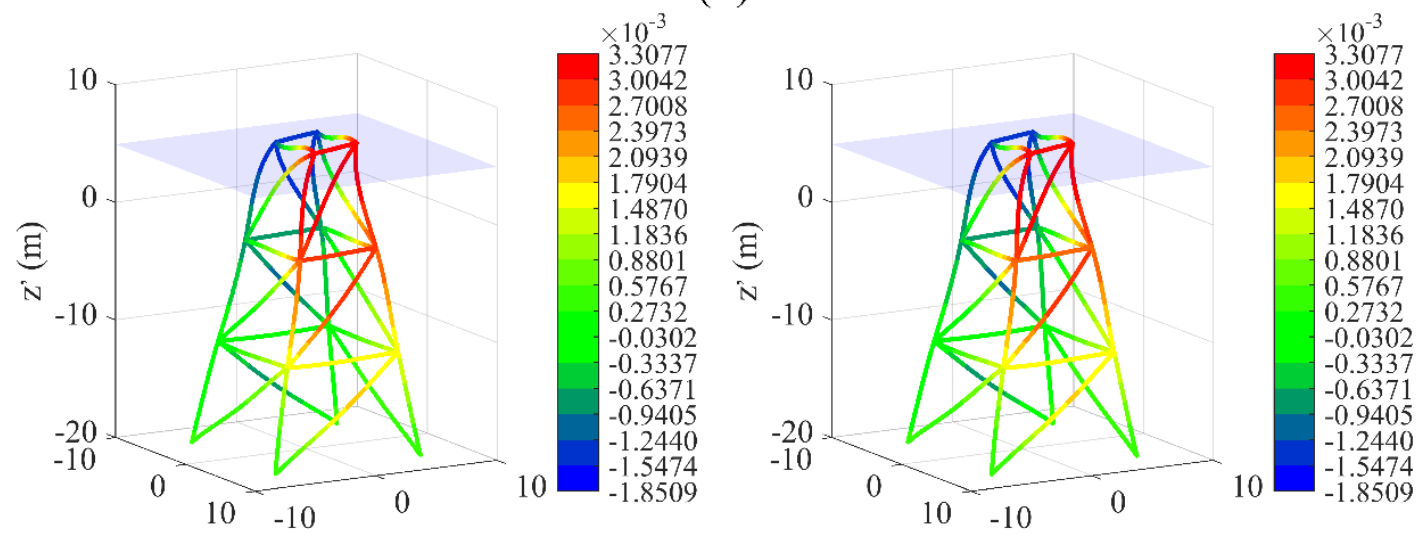

$\mathrm{x}^{\prime}(\mathrm{m}) \quad \mathrm{y}^{\prime}(\mathrm{m})$

(c)

Fig. 25. Variation of displacement $v^{\prime}$ in PD (left), Sesam GeniE (right) at (a) $t=0$, (b) $t=2 T / 6$, and (c) $t=4 T / 6$ (displacements are magnified by 200 for the deformed configurations)

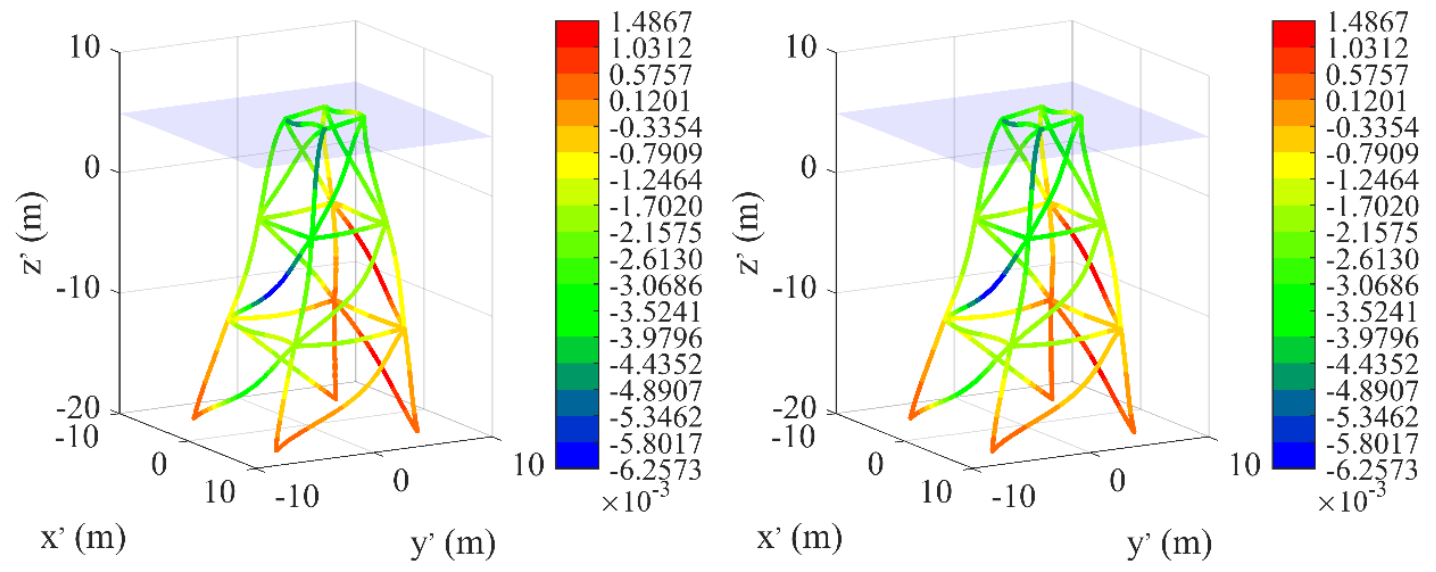

(a) 

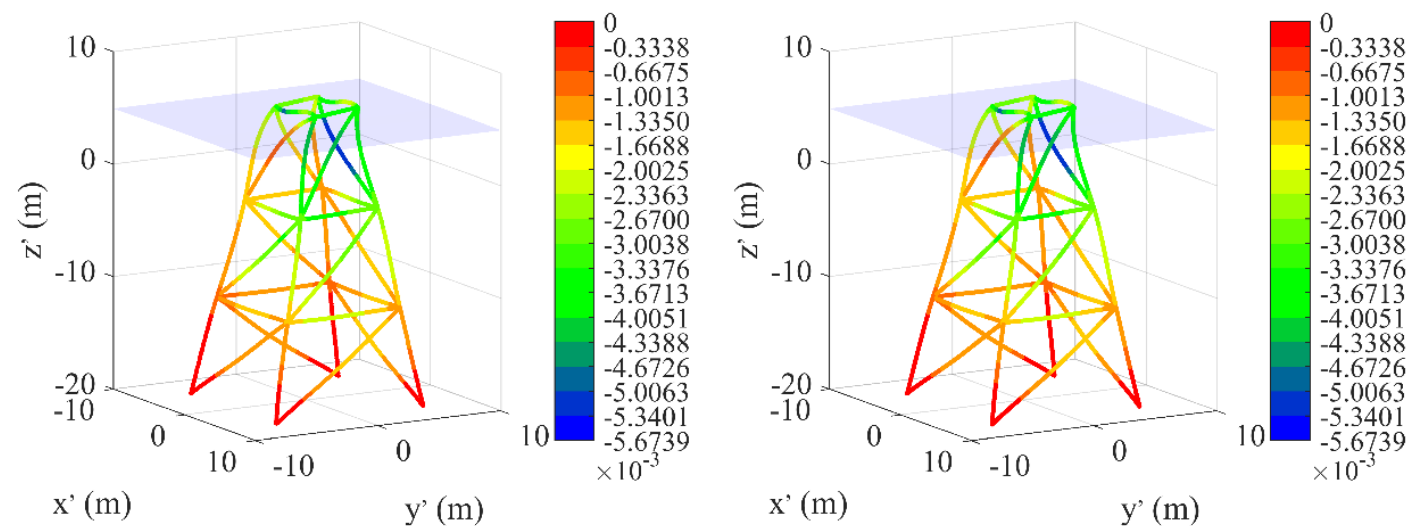

(b)
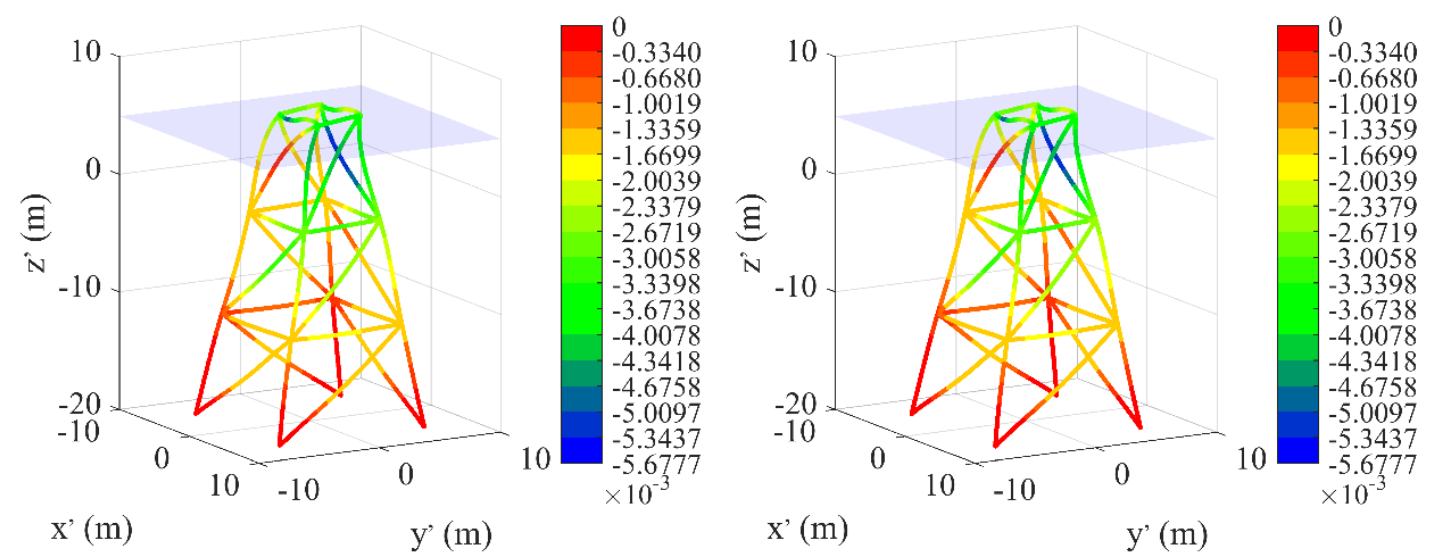

(c)

Fig. 26. Variation of displacement $w^{\prime}$ in PD (left), Sesam GeniE (right) at (a) $t=0$, (b) $t=2 T / 6$, and (c) $t=4 T / 6$ (displacements are magnified by 200 for the deformed configurations)
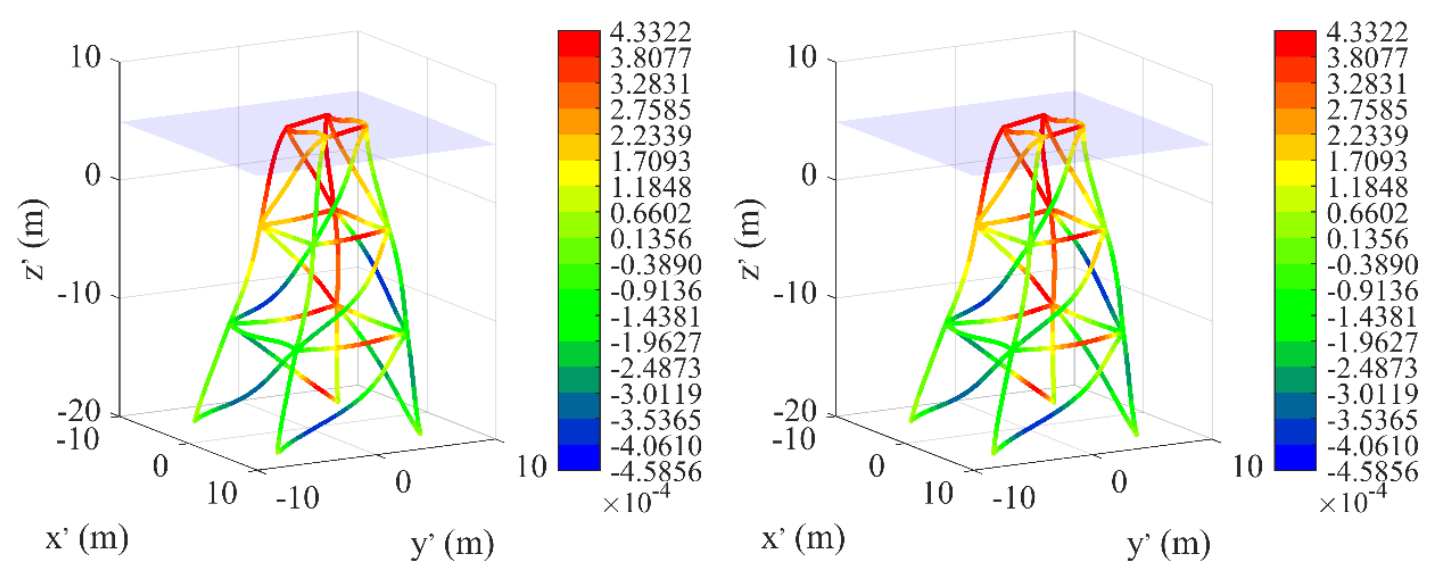

(a) 

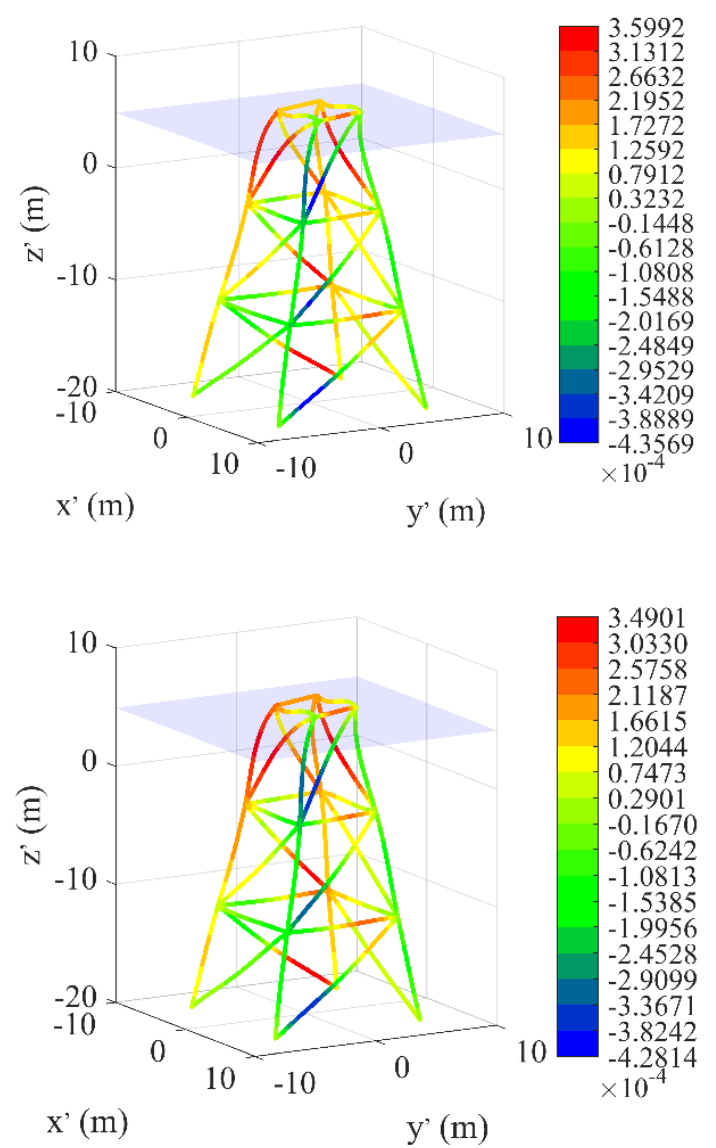

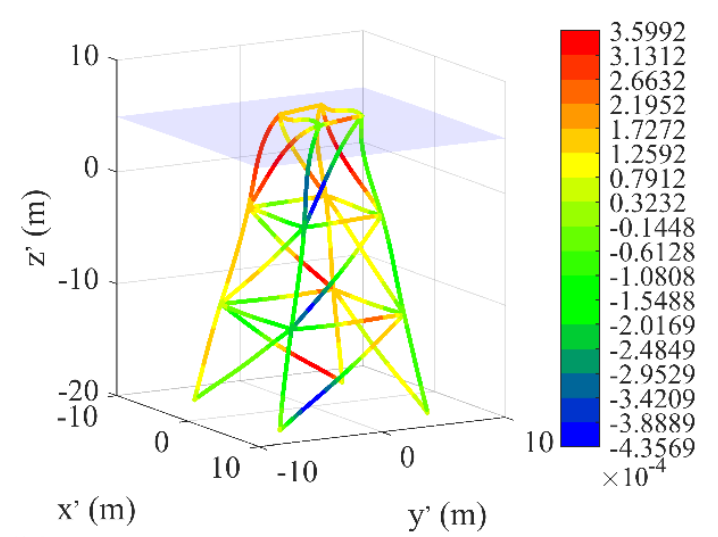

(b)

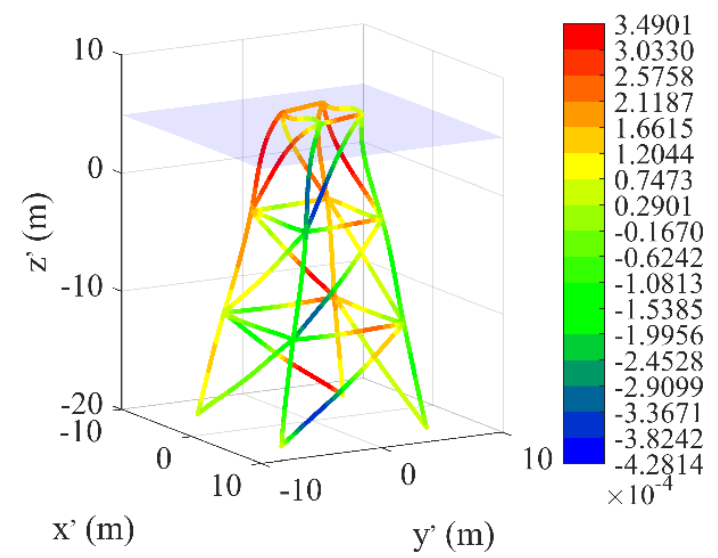

(c)

Fig. 27. Variation of displacement $\theta_{x^{\prime}}$ in PD (left), Sesam GeniE (right) at (a) $t=0$, (b) $t=2 T / 6$, and (c) $t=4 T / 6$ (displacements are magnified by 200 for the deformed configurations)
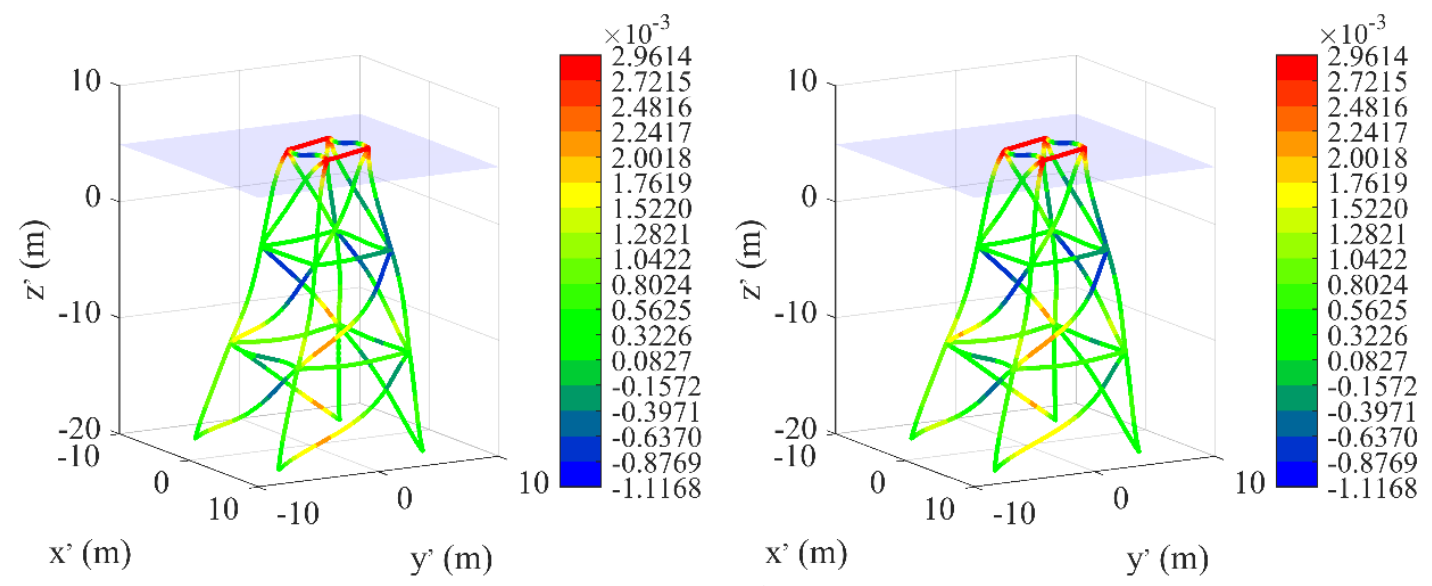

(a) 

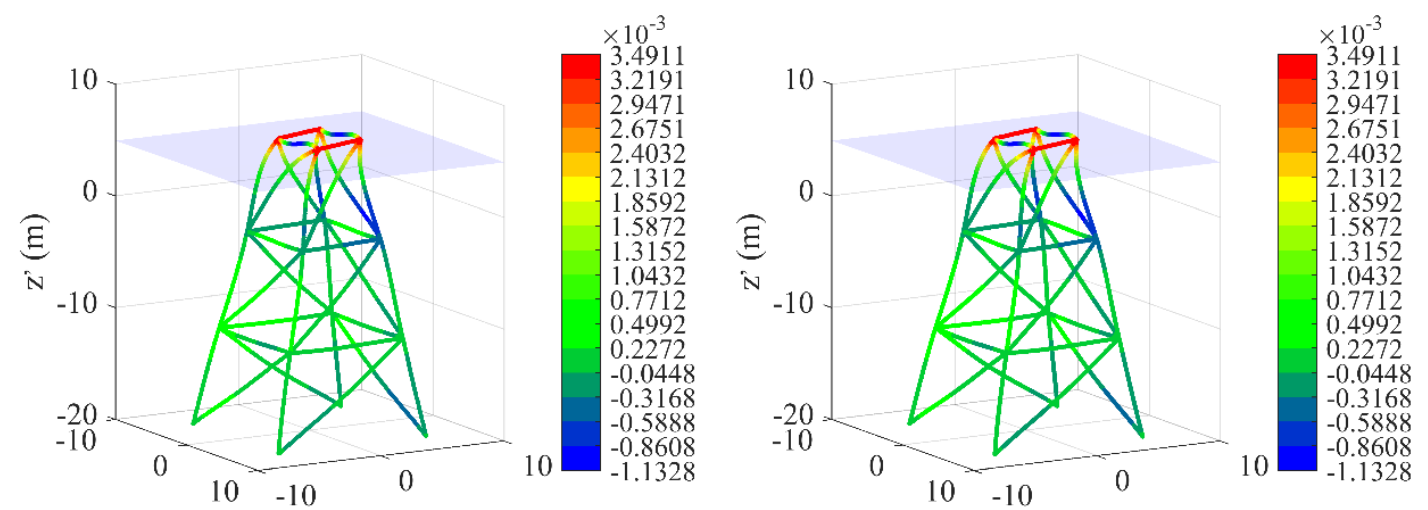

$$
\mathrm{x}^{\prime}(\mathrm{m}) \quad \mathrm{y}^{\prime}(\mathrm{m})
$$

$\mathrm{x}^{\prime}(\mathrm{m})$

(b)
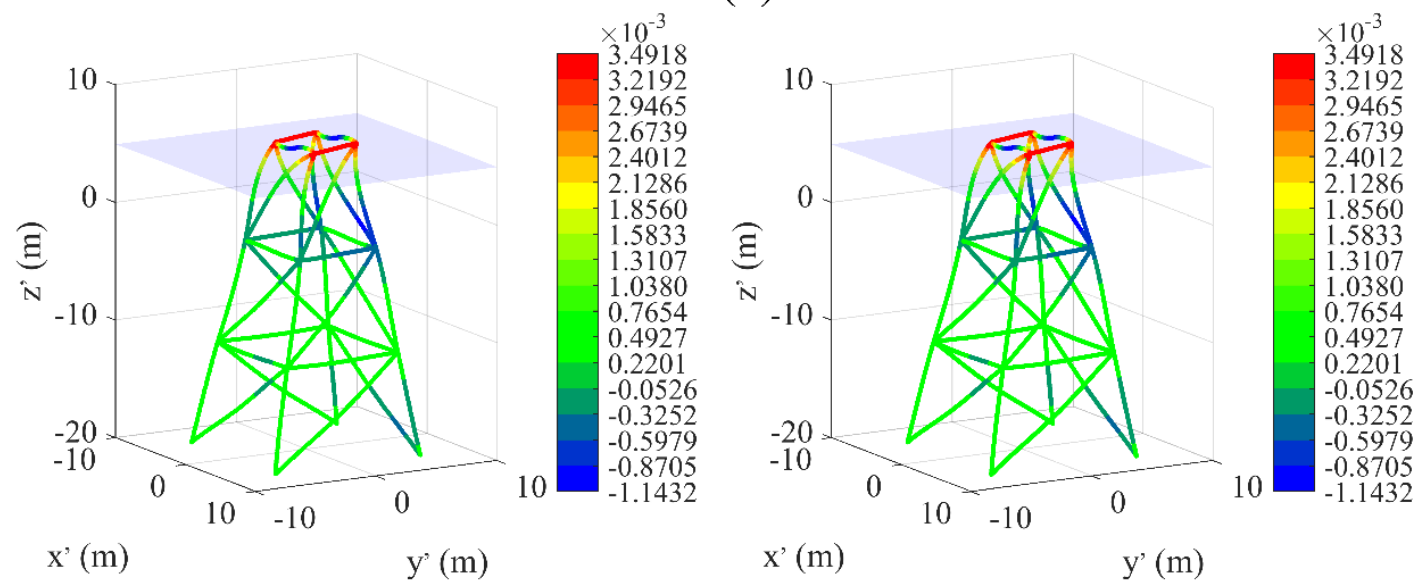

(c)

Fig. 28. Variation of displacement $\theta_{y^{\prime}}$ in $\mathrm{PD}$ (left), Sesam GeniE (right) at (a) $t=0$, (b) $t=2 T / 6$, and (c) $t=4 T / 6$ (displacements are magnified by 200 for the deformed configurations)
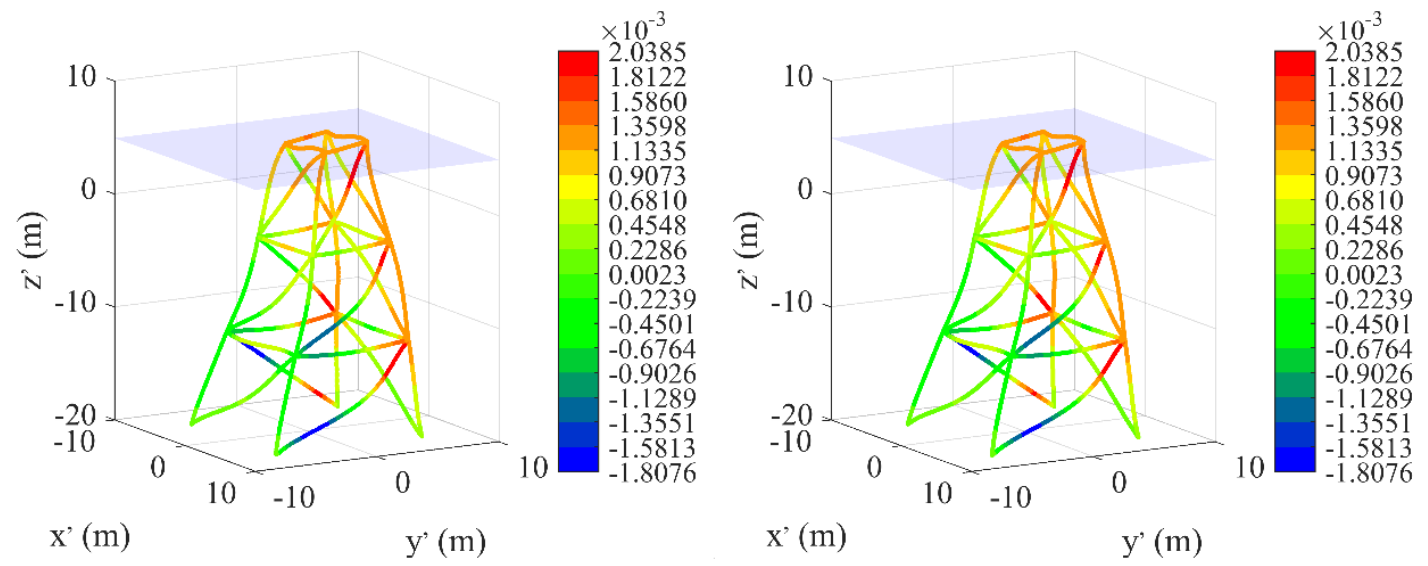

(a) 

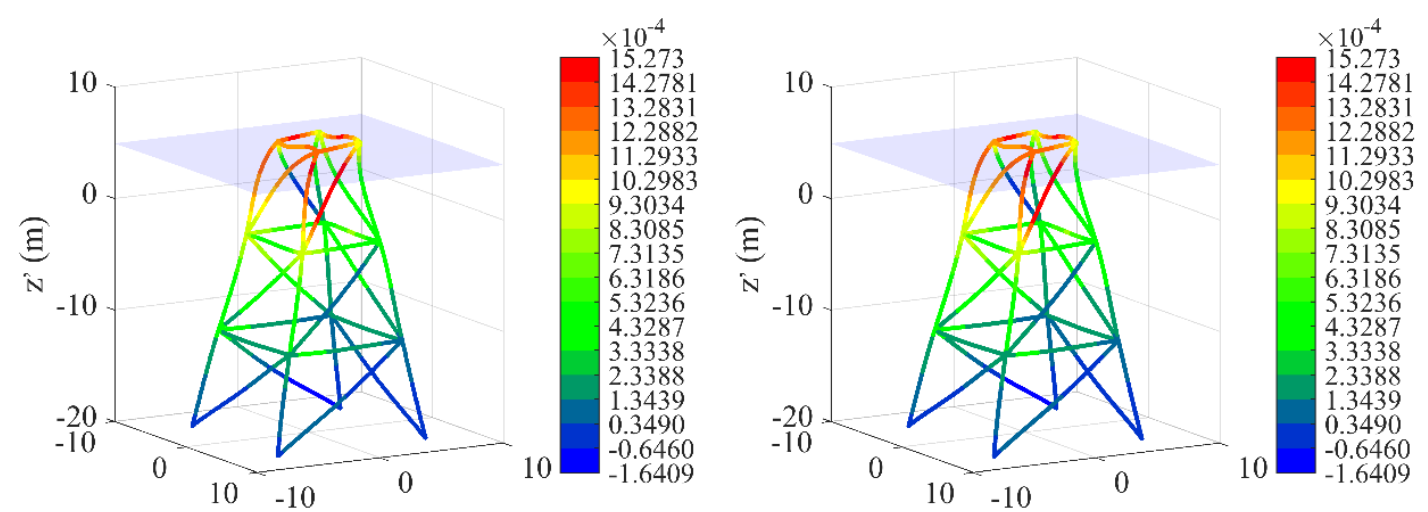

$$
\mathrm{x}^{\prime}(\mathrm{m}) \quad \mathrm{y}^{\prime}(\mathrm{m})
$$

$x^{\prime}(m)$

$y^{\prime}(m)$

(b)
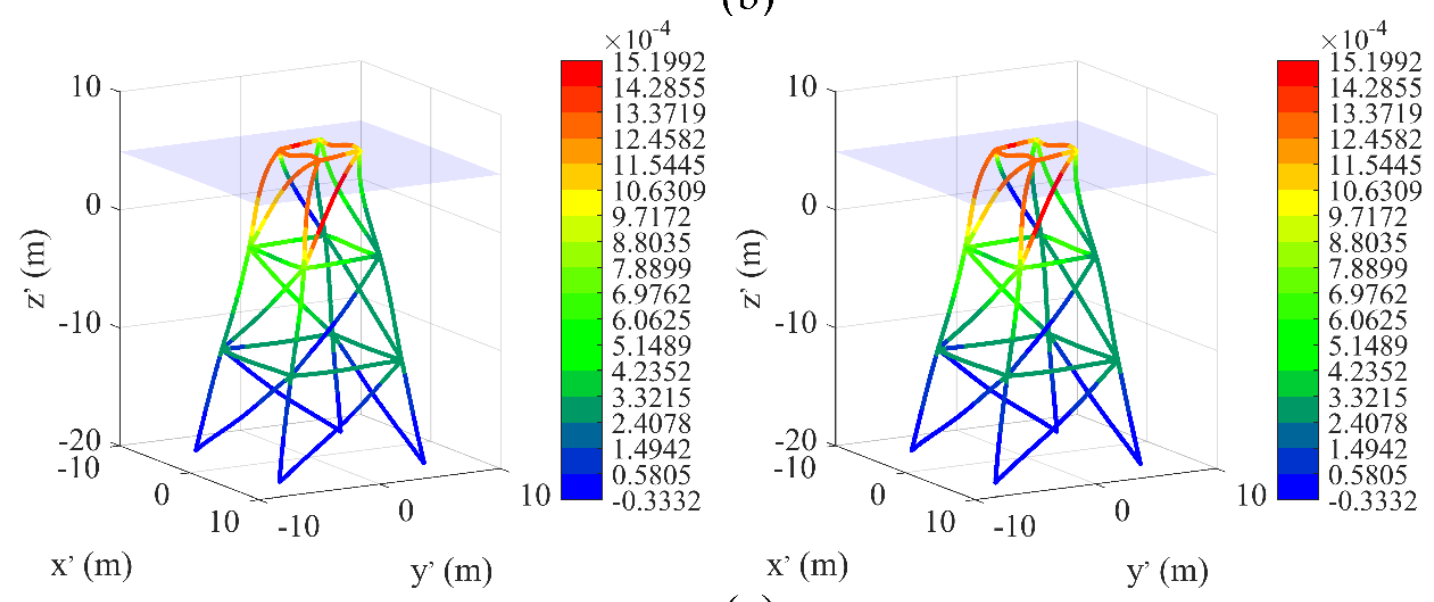

(c)

Fig. 29. Variation of displacement $\theta_{z^{\prime}}$ in PD (left), Sesam GeniE (right) at (a) $t=0$, (b) $t=2 T / 6$, and (c) $t=4 T / 6$ (displacements are magnified by 200 for the deformed configurations)

\subsection{Damage prediction for pre-notched beam subjected to transversely displacement}

After verifying the developed PD model, this example is to predict damage on a pre-notched beam subjected to transversely displacements. As shown in Fig. 30, the beam has length of $L=325 \mathrm{~mm}$ has an initial notch at the middle. The beam has rectangular cross-section with $b=20 \mathrm{~mm}$ and $h=6 \mathrm{~mm}$. The notch height and length are $2 \mathrm{~mm}$ and $3 \mathrm{~mm}$, respectively. The middle section of the beam is applied incremental displacements by using a striking mass as shown in Fig. 30. The beams are made of steel material with Young's modulus $E=2 \times 10^{11} \mathrm{~N} / \mathrm{m}^{2}$, shear modulus $G=1 \times 10^{11} \mathrm{~N} / \mathrm{m}^{2}$, mass density $\rho=7850 \mathrm{~kg} / \mathrm{m}^{2}$, and the critical energy release rate is $G_{c}=720 \mathrm{~J} / \mathrm{m}^{2}$.

In PD model, the beam is discretised into 3250 material points. In order to represent the initial notch, the cross-sectional properties of material points between $L / 2-1.5 \leq x \leq L / 2+1.5 \mathrm{~mm}$ are defined as $b=20 \mathrm{~mm}$ and $h_{2}=4 \mathrm{~mm}$.

In order to apply boundary condition, an incremental transverse displacement at the middle point of the beam is applied as 
$w(x=L / 2, t)=-10^{-8} \times t \mathrm{~m}$

As described in Section 5 for damage prediction, if the energy release rate of an interaction between two material points exceeds the critical value, the interaction is irreversibly removed.

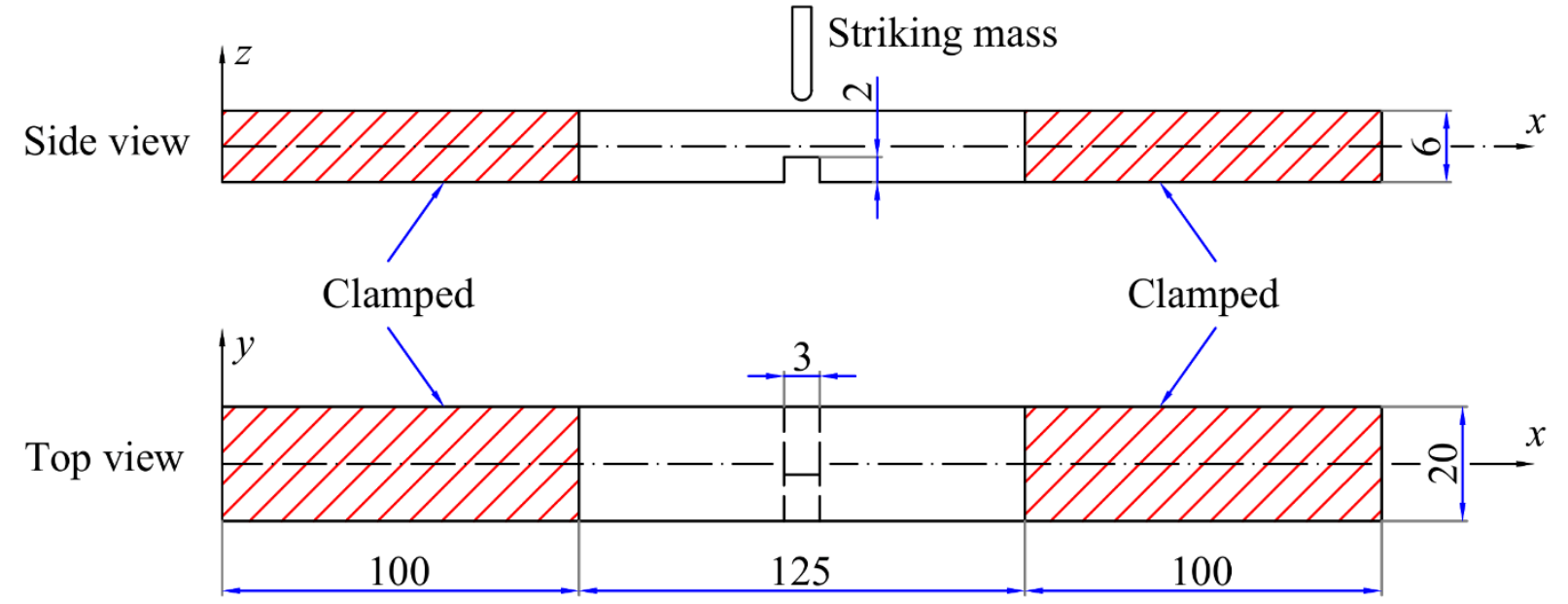

Fig. 30. Pre-notched beam specimen (dimensions are in millimetres)

Fig. 31(a, b) represent the variation of damage coefficient, $\varphi$ for the deformed configuration of the beam obtained by PD. Fig. 31(c) represents the shape of deformed configuration and the damage obtained by an experiment (Villavicencio and Soares, 2012). As shown in Fig. 31(b), the beam is completely split when the displacement of the middle point reaches $w(L / 2)=-2.5 \times 10^{-4} \mathrm{~m}$. It can also be seen that the damage location and the deformed shape of the beam captured by the developed PD model agree very well with experimental result. 


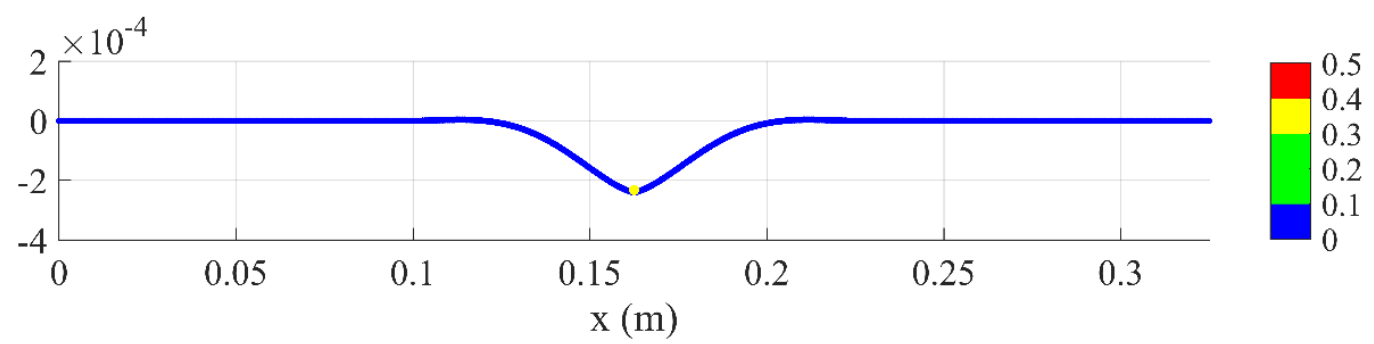

(a)

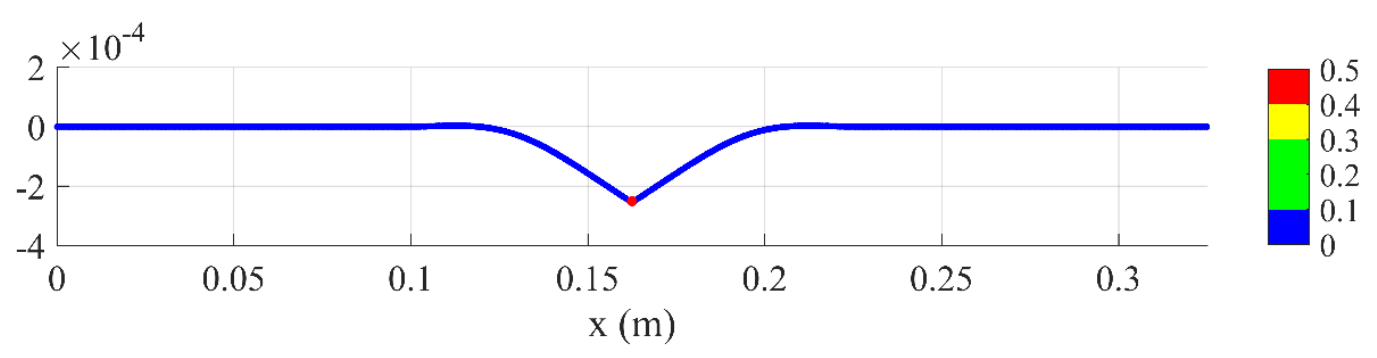

(b)

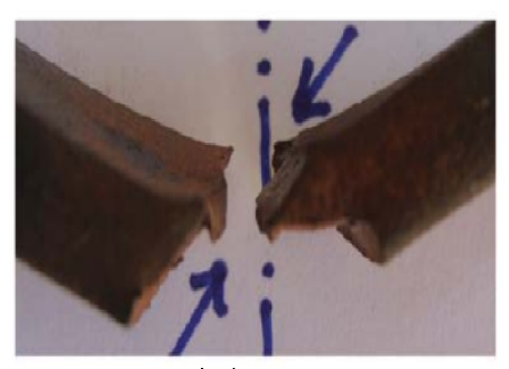

(c)

Fig. 31. Damage coefficient $\varphi$ in deformed configuration when (a) $w(L / 2)=-2.4 \times 10^{-4} \mathrm{~m}$, (b) $w(L / 2)=-2.5 \times 10^{-4} \mathrm{~m}$, (c) experiment (Villavicencio and Soares, 2012)

7.4. Damage prediction for jacket platform subjected to sea loads

After verifying the developed PD model for transient analysis of jacket platform, damage prediction for jacket platform subjected to sea loads is investigated. In this example, the jacket platform described in Section 7.1.3 has an initial damage at $z_{4}^{\prime}=13.5 \mathrm{~m}$ as shown in Fig. 32 and it is subjected to sea loads. The sea loads and material properties are described in Section 7.2. The critical energy release rate is $G_{c}=720 \mathrm{~J} / \mathrm{m}^{2}$. 


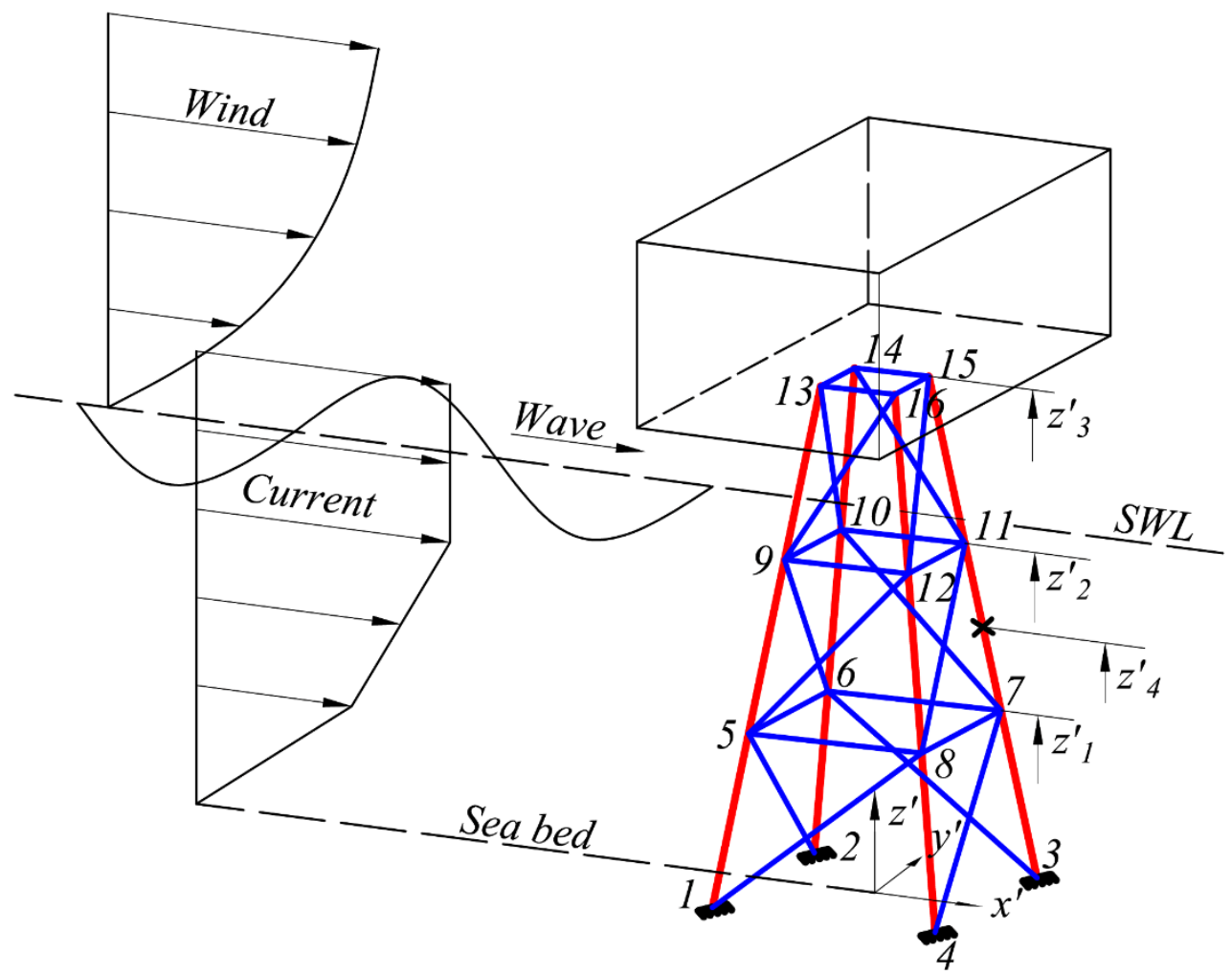

Fig. 32. Initial damaged jacket platform and the sea condition

Figs. 33-37 represent the variation of damage coefficient $\varphi$ on the jacket platform at different times. For visualization, the damaged positions are zoomed out. In Figs. 33-37, P5 - P16 represent joint point numbers as described in Fig. 15b. It is observed that the structure is damaged around joint points P5 to P12 after 1.875 seconds which causes discontinuities between beams as shown in Fig. 33. The damage positions remain the same after 15 seconds but the gap between discontinuities become larger as shown in Figs. 34-35. After 28.125 seconds, the structure is also damaged around joint points $\mathrm{P} 3$ and P16 as shown in Fig. 37. As it can be observed from the figure, the structure is mostly damaged due to applied loading. In conclusion, the damage starts and develops around joint positions. Therefore, the structure needs to be strengthened at these positions in order to minimize the occurrence of damage. 


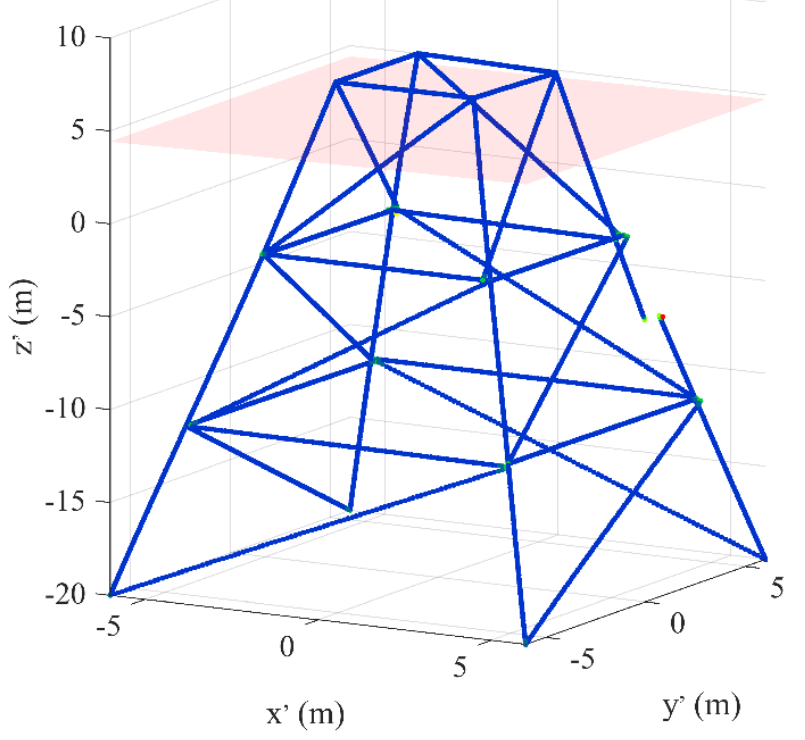

(P5)

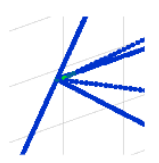

(P6)

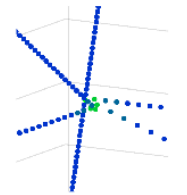

(P7)

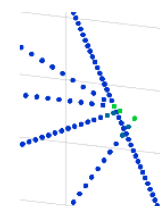

(P8)

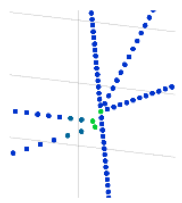

(P9)

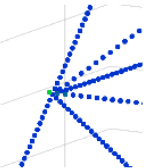

(P10)

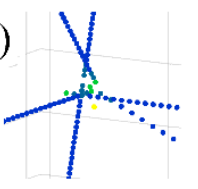

(P11)

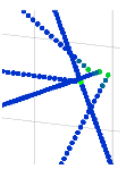

(P12)

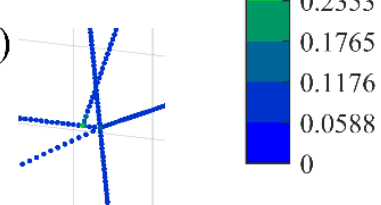

Fig. 33. Damage coefficient $\varphi$ in deformed configuration at $t=1.875 \mathrm{~s}$

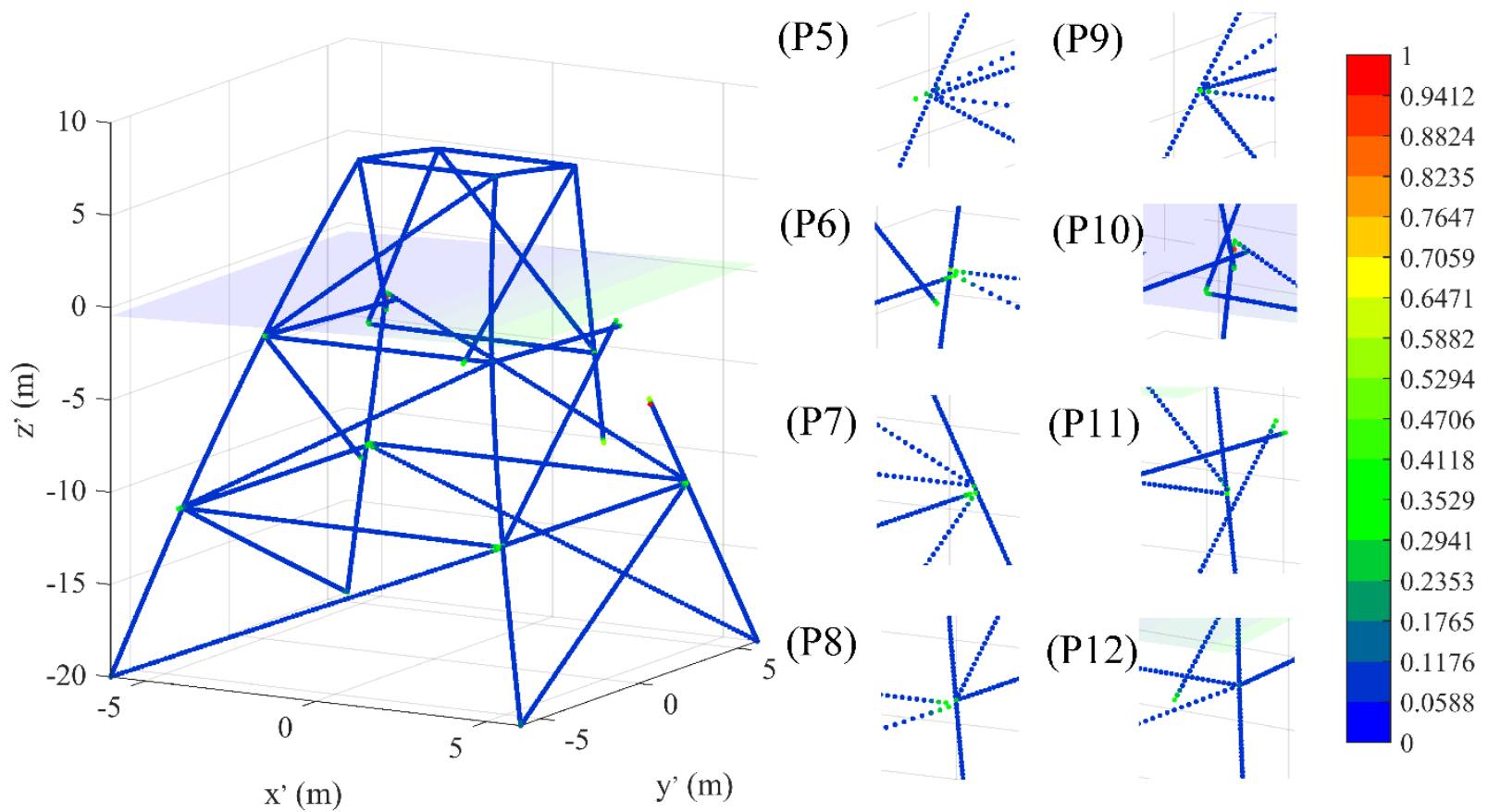

Fig. 34. Damage coefficient $\varphi$ in deformed configuration at $t=7.5 \mathrm{~s}$ 


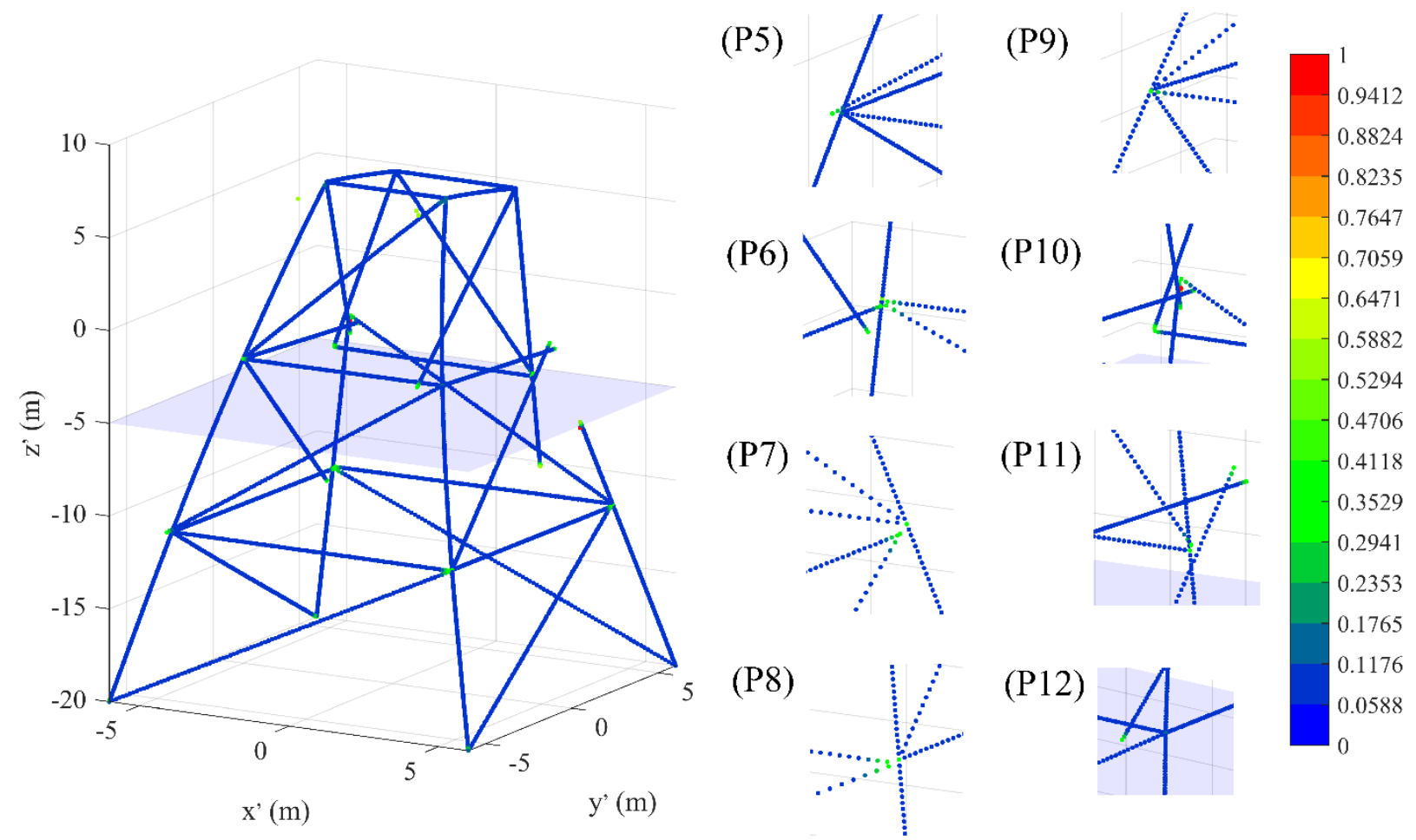

Fig. 35. Damage coefficient $\varphi$ in deformed configuration at $t=15 \mathrm{~s}$

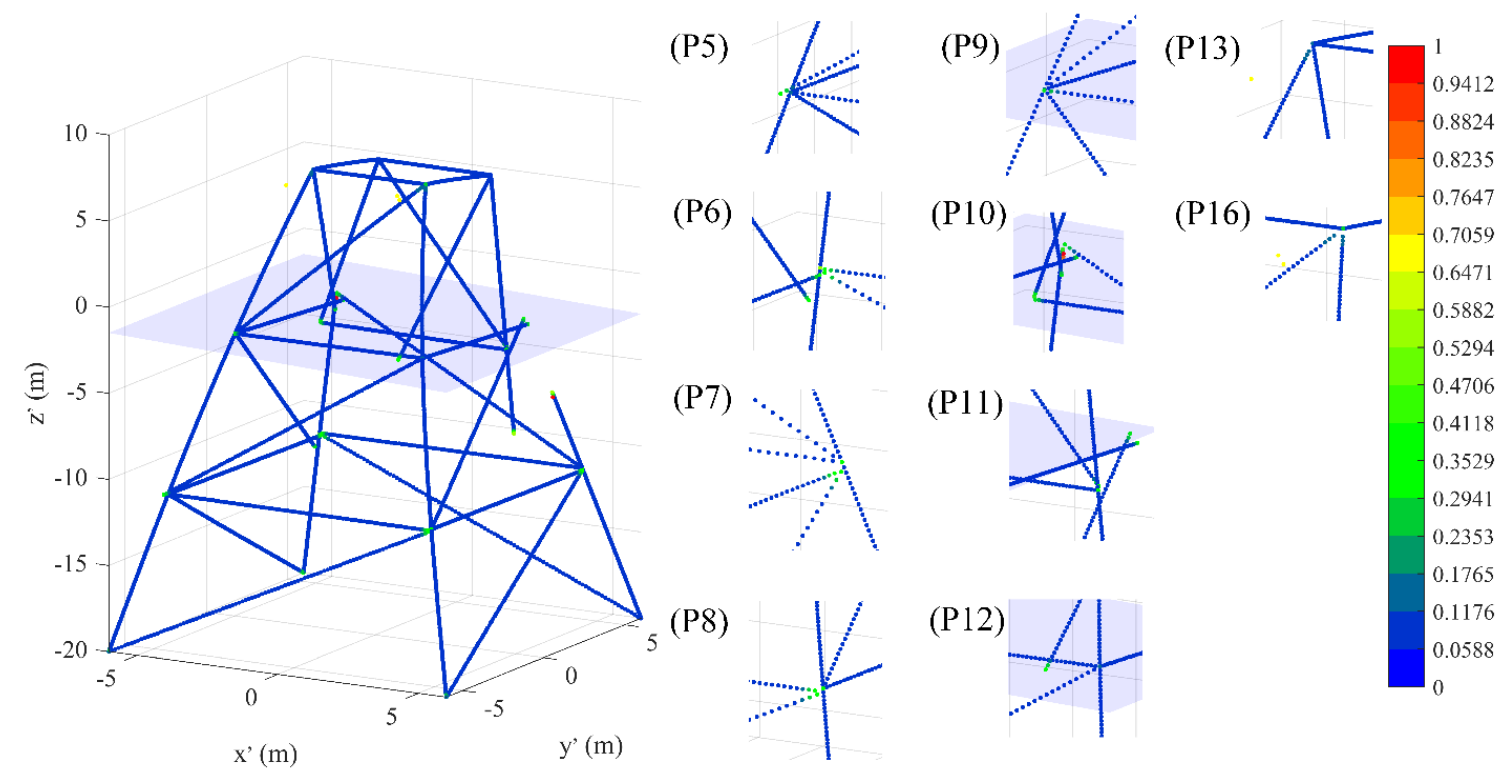

Fig. 36. Damage coefficient $\varphi$ in deformed configuration at $t=20.625 \mathrm{~s}$ 


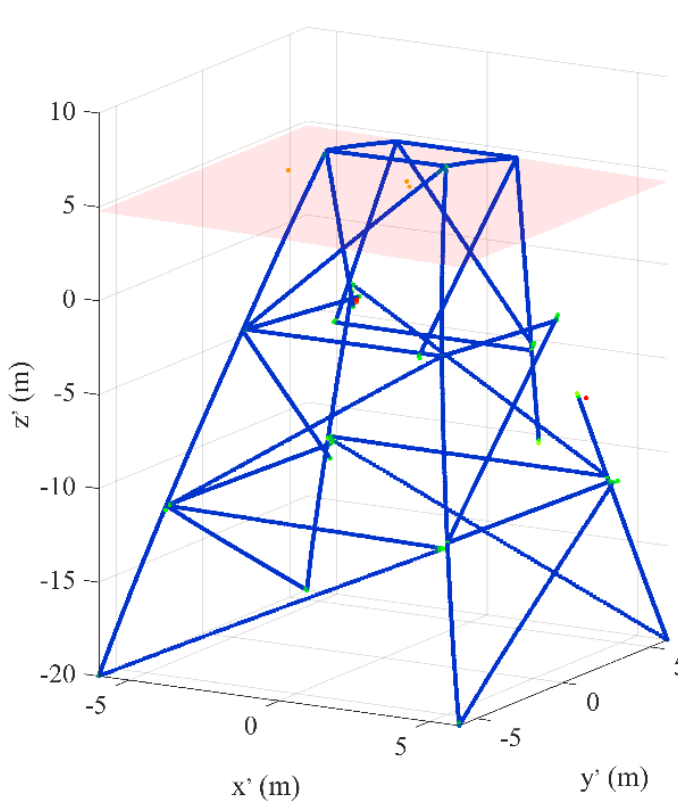

(P5)

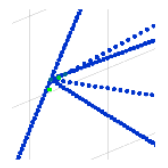

(P6)

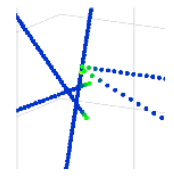

(P7)

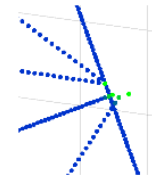

(P8)

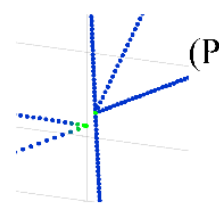

(P9)

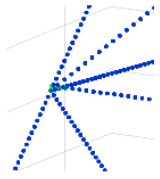

(P10)

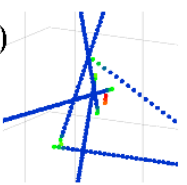

(P14)

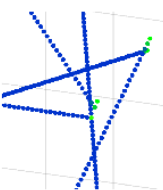

P12)

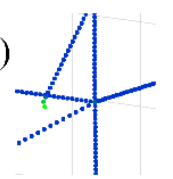

(P13)

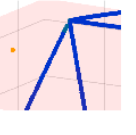

$-0.8824$

0.8235

0.7647

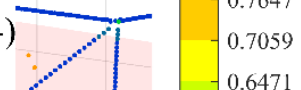

0.6471

0.5882

0.5294

0.4706

0.4118

0.3529

0.2941

0.2353

0.1765

0.1176

0.0588

Fig. 37. Damage coefficient $\varphi$ in deformed configuration at $t=28.125 \mathrm{~s}$

\subsection{Damage prediction for jacket platform subjected to ship collision}

In this example, the jacket platform subjected to ship collision is investigated. The ship is considered as a rigid body, moving with a constant velocity of $v_{0}=30 \mathrm{~m} / \mathrm{s}$ in $x^{\prime}$ direction. It is assumed that the collision is in between the front part of the ship and the jacket platform. Therefore, only the front part of the ship is modeled as shown in Fig. 38. The geometrical and material properties of the jacket platform are the same as in Section 7.2. The structure does not have any initial damage, but it is hit by the ship at $t=0$. The critical energy release rate of the material is $G_{c}=720 \mathrm{~J} / \mathrm{m}^{2}$.

Fig. 38 presents the extent of damage due to contact between ship and the jacket platform. As the ship moves to the left, the ship growingly collides with the jacket platform causing the increase of damage region on jacket platform. As shown in Fig. 38, the deformed shape of the contact region on the jacket platform is also associated with the shape of the contact region on the ship body. After 0.15 seconds, the ship with the velocity of $v_{0}=30 \mathrm{~m} / \mathrm{s}$ moves $0.45 \mathrm{~m}$ and breaks the jacket platform on contact regions. 


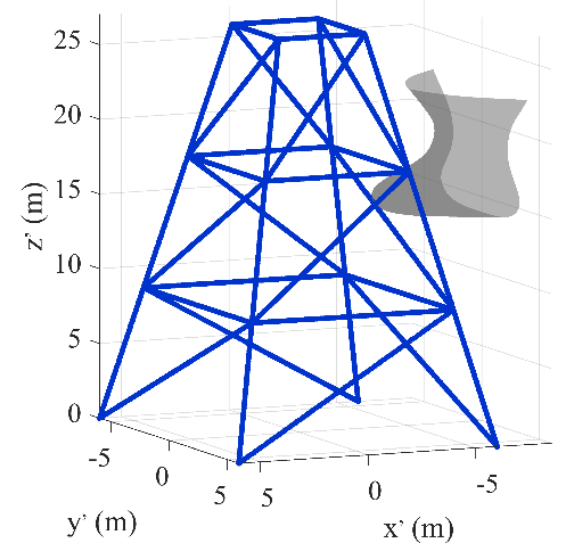

(a)

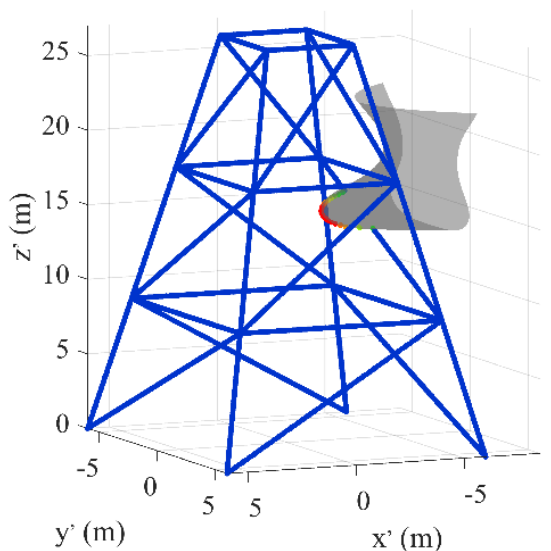

(c)

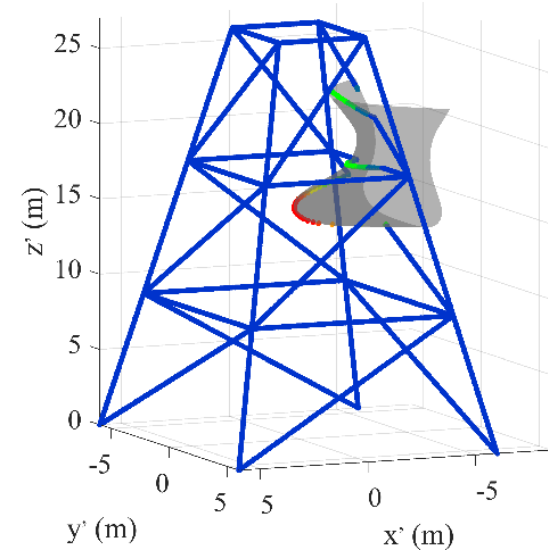

(e)

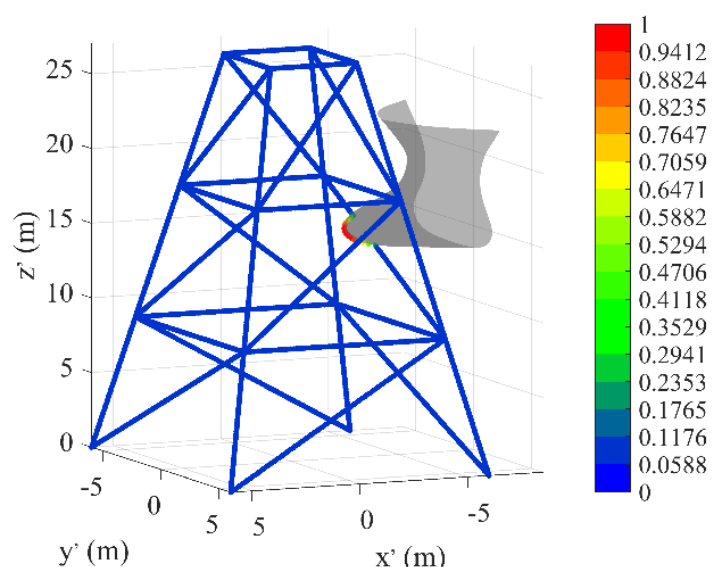

(b)

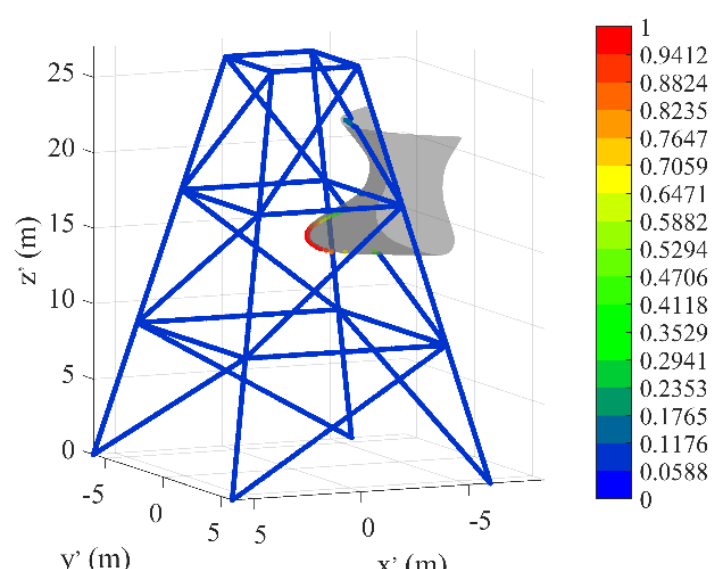

(d)

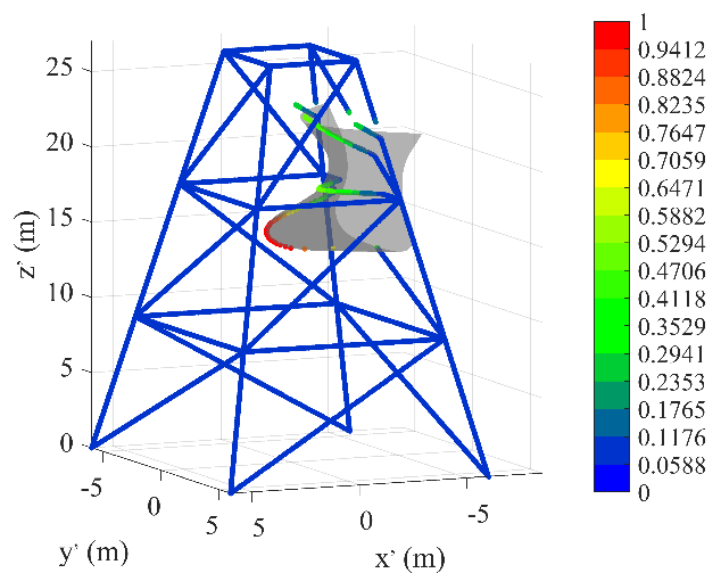

(f)

Fig. 38. Damage coefficient $\varphi$ in deformed configuration at (a) $t=0$, (b) $t=0.03 \mathrm{~s}$, (c)

$$
t=0.06 \mathrm{~s} \text {, (d) } t=0.09 \mathrm{~s} \text {, (e) } t=0.12 \mathrm{~s} \text {, (f) } t=0.15 \mathrm{~s}
$$

\section{Concluding remarks}

This study provides a novel PD approach for dealing with complex beam structures. For this purpose, a bond based PD beam model with 6 degrees of freedom is developed. The developed 
PD model is tested with various beam structures including straight and curved beams. The numerical procedure for investigating complex beam structures is also introduced for the first time in the PD literature. The results from PD analyses in both static and transient analyses have good agreement with those in FEA solutions. Damage criteria for beam model based on critical energy release rate are provided for damage predictions. The developed PD model is used to predict the damage for a pre-notched beam subjected to transverse displacements, a jacket platform subjected to sea load as well as ship-offshore jacket platform collision. The developed PD model can be used for any type of beam structures to predict possible damages that may occur during the operation process. 


\section{Acknowledgment}

The authors gratefully acknowledge financial support from the Ministry of Education and Training in Vietnam and University of Strathclyde.

\section{Appendix A. Linear wave theory}

Wave theories can be classified into two families which are "small amplitude wave theories" and "long wave theories" (Le Mehaute, 1969). The small amplitude wave theories are the linearized theories of the first categories of power series (Mader, 2004). The long wave theories use nonlinear long wave equations. Wave theories also depend on the wave height, wave length, wave period, and water depth as described by Le Mehaute (1969). In this study, for simplification, the linear wave (airy) theory is used.

\section{A.l Wave surface}

According to linear wave theory (Veritas, 1993) wave surface is sinusoidal as represented in Fig. A.1. The wave elevation at any point on the wave surface can be represented as

$$
\boldsymbol{\eta}\left(\mathbf{x}_{s}^{\prime}, t\right)=A_{w} \cos \left(\mathbf{k} \cdot \mathbf{x}_{s}^{\prime}-\omega t\right)
$$

where $A_{w}$ represents the wave amplitude which is equal to half of the wave height, $\mathbf{k}$ represents two-dimensional wave number, $\omega$ represents the wave angular frequency, $t$ represents the time and $\mathbf{x}_{s}^{\prime}$ represents a point on the wave surface which can be written as

$\mathbf{x}_{s}^{\prime}=x_{s}^{\prime} \cdot \mathbf{i}_{1}+y_{s}^{\prime} \cdot \mathbf{i}_{2}$

Two-dimensional wave number, $\mathbf{k}$ can be represented in terms of direction of wave propagation, $\beta$ as

$\mathbf{k}=k\left(\mathbf{i}_{1} \cos \beta+\mathbf{i}_{2} \sin \beta\right)$

where $k$ represents the absolute value of wave number which can be calculated from wave length, $L$ as

$k=\frac{2 \pi}{L}$

Wave angular frequency, $\omega$ can be calculated from wave period, $T$, by using following relation $\omega=\frac{2 \pi}{T}$ 


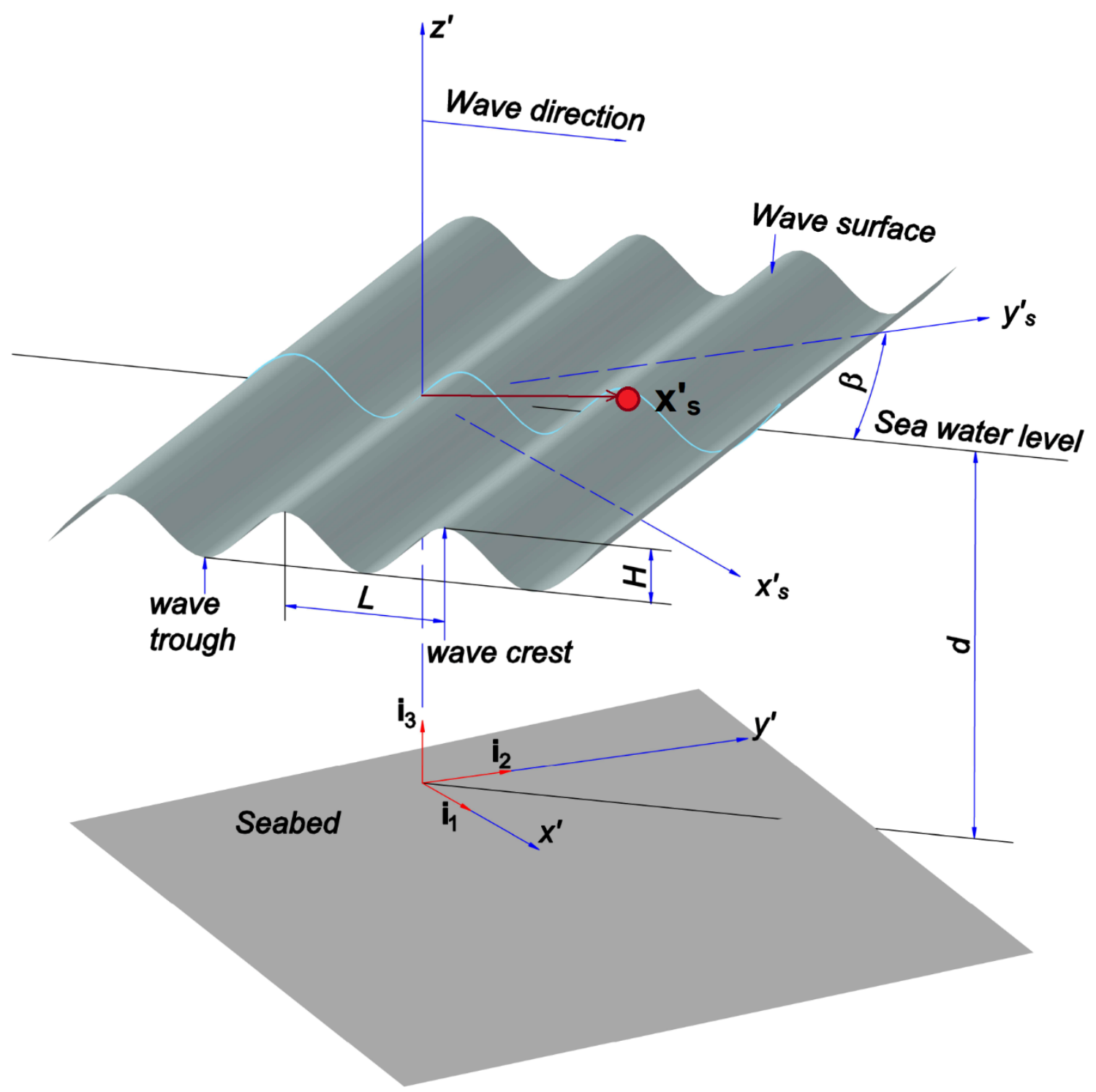

Fig. A1. Periodic wave surface

\section{A.2 Deep water kinematics}

When $d / L>0.5$, the environment is considered as deep water (Andersen et al., 2014). The wave length can be calculated as (Guo, 2002)

$$
L=\frac{2 \pi d}{\bar{x}^{2}\left(1-e^{-\bar{x}^{2.4908}}\right)^{-1 / 2.4908}}
$$

where $\bar{x}=\omega d / \sqrt{g d}$, and $g$ represents gravitational acceleration.

The undisturbed fluid velocity can be calculated as (Veritas, 1993)

$\mathbf{v}_{\text {(wave) }}^{\prime}=\mathrm{v}_{x^{\prime}(\text { wave })} \mathbf{i}_{1}+\mathrm{v}_{\left.y^{\prime} \text { (wave }\right)} \mathbf{i}_{2}+\mathrm{v}_{\left.z^{\prime} \text { (wave }\right)} \mathbf{i}_{3}$

with

$\mathrm{v}_{x^{\prime}(\text { wave })}=A_{w} \omega \cos \beta \frac{\cosh \left(k\left(z^{\prime}+d\right)\right)}{\sinh (k d)} \cos \left(\mathbf{k} \cdot \mathbf{x}_{s}^{\prime}-\omega t\right)$ 


$$
\begin{aligned}
& \mathrm{v}_{y^{\prime}(\text { wave })}=A_{w} \omega \sin \beta \frac{\cosh \left(k\left(z^{\prime}+d\right)\right)}{\sinh (k d)} \cos \left(\mathbf{k} \cdot \mathbf{x}_{s}^{\prime}-\omega t\right) \\
& \mathrm{v}_{z^{\prime}}=A_{w} \omega \frac{\sinh \left(k\left(z^{\prime}+d\right)\right)}{\sinh (k d)} \sin \left(\mathbf{k} \cdot \mathbf{x}_{s}^{\prime}-\omega t\right)
\end{aligned}
$$

The undisturbed fluid acceleration can be calculated as (Veritas, 1993)

$$
\mathbf{a}_{\text {(wave) }}^{\prime}=\mathrm{a}_{x^{\prime}(\text { wave })} \mathbf{i}_{1}+\mathrm{a}_{y^{\prime}(\text { wave })} \mathbf{i}_{2}+\mathrm{a}_{z^{\prime}(\text { wave })} \mathbf{i}_{3}
$$

with

$$
\begin{aligned}
& \mathrm{a}_{x^{\prime}(\text { wave })}=A_{w} \omega^{2} \cos \beta \frac{\cosh \left(k\left(z^{\prime}+d\right)\right)}{\sinh (k d)} \sin \left(\mathbf{k} \cdot \mathbf{x}_{s}^{\prime}-\omega t\right) \\
& \mathrm{a}_{y^{\prime}(\text { wave })}=A_{w} \omega^{2} \sin \beta \frac{\cosh \left(k\left(z^{\prime}+d\right)\right)}{\sinh (k d)} \sin \left(\mathbf{k} \cdot \mathbf{x}_{s}^{\prime}-\omega t\right) \\
& \mathrm{a}_{z^{\prime}}=-A_{w} \omega^{2} \frac{\sinh \left(k\left(z^{\prime}+d\right)\right)}{\sinh (k d)} \cos \left(\mathbf{k} \cdot \mathbf{x}_{s}^{\prime}-\omega t\right)
\end{aligned}
$$

The horizontal velocity can be calculated as (Veritas, 1993) $\mathbf{v}_{h(\text { wave })}^{\prime}=\mathrm{v}_{x^{\prime}(\text { wave })} \mathbf{i}_{1}+\mathrm{v}_{y^{\prime}(\text { wave })} \mathbf{i}_{2}$

Note that above the still water, the velocity distribution at the wave crest can be assumed equal to the water velocity at the still water level as shown in Fig. A2 (Faltinsen, 1993).

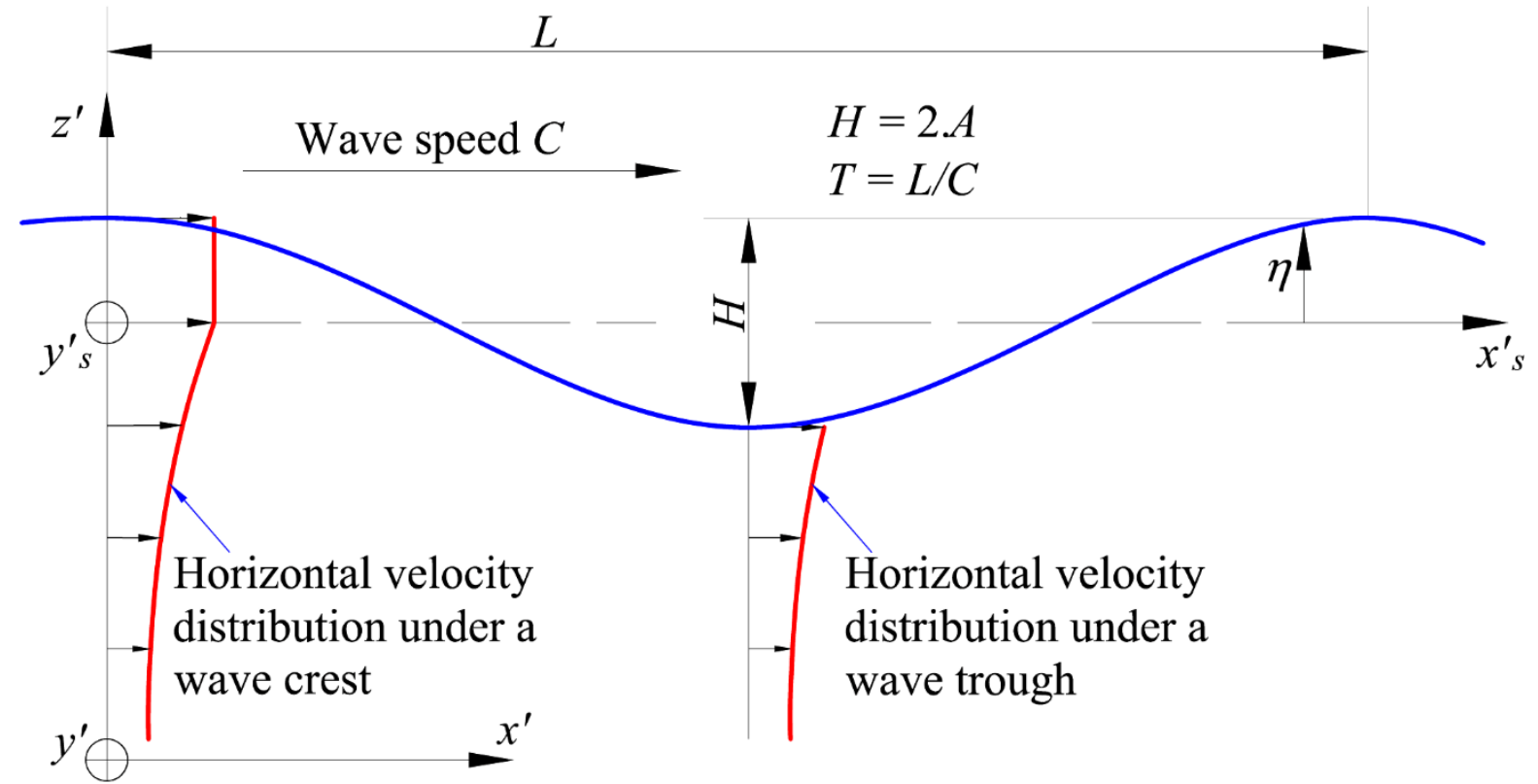

Fig. A2. Velocity distribution at wave crest and wave trough

\section{A.3 Shallow water kinematics}

When $d / L<1 / 20$, the environment is considered as shallow water (Le Mehaute et al., 1969) and the wave length and the absolute wave number can be defined as (Andersen et al., 2014) 
$L=T \sqrt{g d}$

For shallow water $k\left(z^{\prime}+d\right) \rightarrow 0 \quad$ and $\quad k d \rightarrow 0, \quad$ therefore $\cosh \left(k\left(z^{\prime}+d\right)\right) \rightarrow 1$, $\sinh \left(k\left(z^{\prime}+d\right)\right) \rightarrow k\left(z^{\prime}+d\right)$, and $\sinh (k d) \rightarrow k d$

Therefore, the fluid velocity given in Eq. (A.7) reduces to

$\mathrm{v}_{x^{\prime}(\text { wave })}=A \omega \cos \beta \frac{1}{k d} \cos \left(\mathbf{k x}_{s}^{\prime}-\omega t\right)$

$\mathrm{v}_{y^{\prime}(\text { wave })}=A \omega \sin \beta \frac{1}{k d} \cos \left(\mathbf{k x}_{s}^{\prime}-\omega t\right)$

$\mathrm{v}_{z^{\prime}(\text { wave })}=A \omega \frac{z^{\prime}+d}{d} \sin \left(\mathbf{k x}_{s}^{\prime}-\omega t\right)$

The fluid acceleration given in Eq. (A.8) reduces to

$$
\begin{aligned}
& \mathrm{a}_{x^{\prime}(\text { wave })}=A \omega^{2} \cos \beta \frac{1}{k d} \sin \left(\mathbf{k} \mathbf{x}_{s}^{\prime}-\omega t\right) \\
& \mathrm{a}_{y^{\prime}(\text { wave })}=A \omega^{2} \sin \beta \frac{1}{k d} \sin \left(\mathbf{k} \mathbf{x}_{s}^{\prime}-\omega t\right) \\
& \mathrm{a}_{z^{\prime}(\text { wave })}=-A \omega^{2} \frac{z^{\prime}+d}{d} \cos \left(\mathbf{k} \mathbf{x}_{s}^{\prime}-\omega t\right)
\end{aligned}
$$

A.4 Wave combined with current

The current velocity and acceleration can be defined as

$$
\begin{aligned}
\mathbf{v}_{\text {(current) }}^{\prime} & =\mathrm{v}_{x^{\prime}(\text { current })} \mathbf{i}_{1}+\mathrm{v}_{y^{\prime}(\text { current })} \mathbf{i}_{2}+\mathrm{v}_{z^{\prime}(\text { current })} \mathbf{i}_{3} \\
\mathbf{a}_{\text {(current })}^{\prime} & =\mathbf{0}
\end{aligned}
$$

The total fluid velocity and acceleration caused by wave and current can be calculated as $\mathbf{v}_{(\text {fluid })}^{\prime}=\mathbf{v}_{(\text {current })}^{\prime}+\mathbf{v}_{(\text {wave })}^{\prime}$

$\mathbf{a}_{(\text {fluid })}^{\prime}=\mathbf{a}_{(\text {wave })}^{\prime}$

\section{Appendix B. Loading calculation}

As shown in Fig. B1, the applied load on an offshore beam structure includes gravitational, buoyancy, wave, current and wind loads. The beam is discretized into material points. The loading acting on each material is summarised as:

\section{B1. Gravitational loads}

The gravitational force only have a component in the $-z^{\prime}$ direction which can be calculated as $F_{\text {gravity }(k)}=g \rho V_{(k)}$

where $V_{(k)}$ represents the volume of material point $k$, and $\rho$ represents the mass density of the material.

\section{B2. Buoyancy loads}

The buoyancy forces only have a component in the $+z^{\prime}$ direction which can be calculated as $F_{\text {buoyancy }(k)}=g \rho_{(\text {fluid })} V_{\text {displaced }(k)}$

where $V_{\text {displaced }(k)}$ represents the volume of fluid displaced by material point $k$, and $\rho_{(\text {fluid })}$ 
represents the mass density of the fluid. Note that in this study $V_{\text {displaced }(k)}=\pi D_{\text {out }}^{2} d x / 4$ for each material point, where $D_{\text {out }}$ represents outer diameter of the beam.

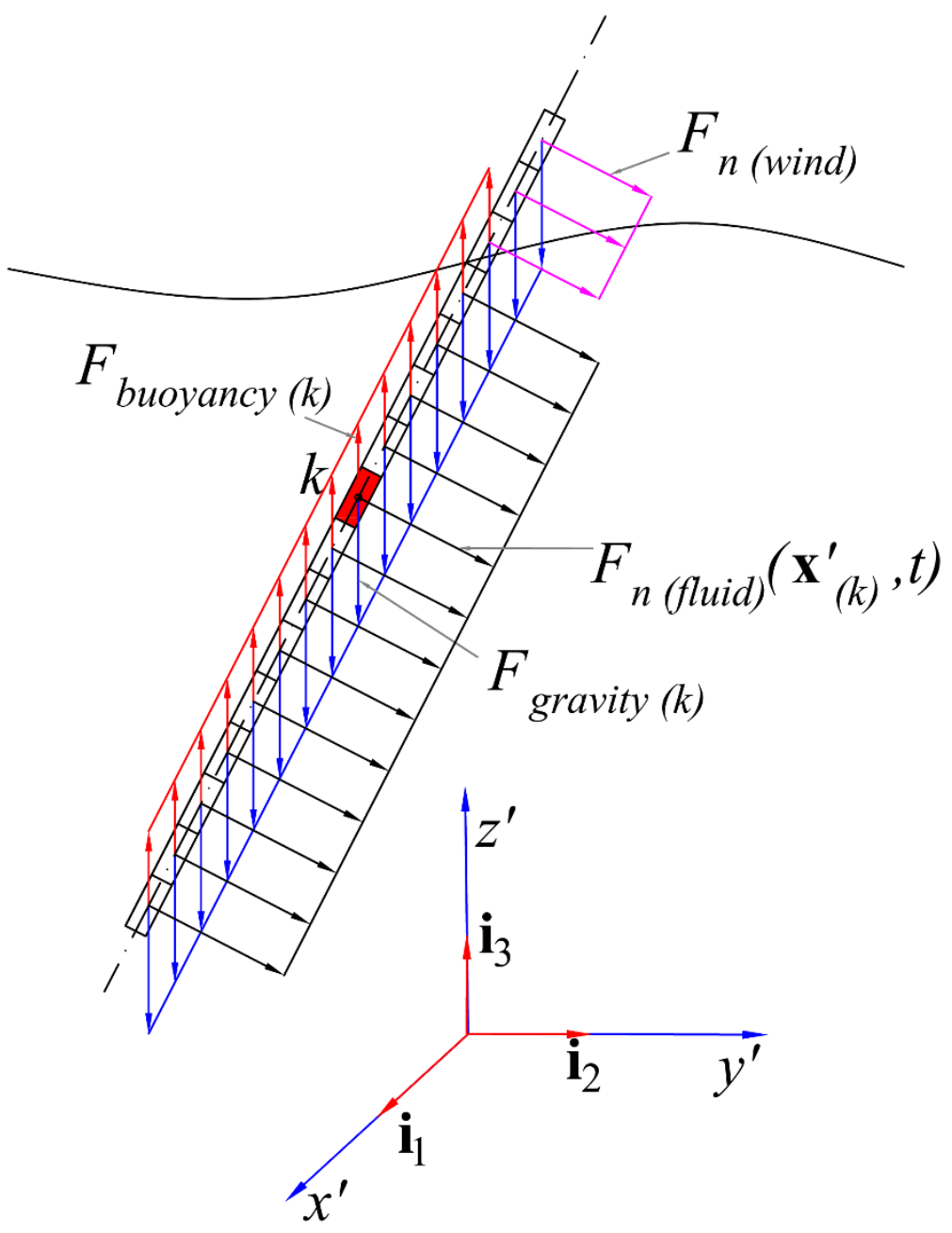

Fig. B1. Components of sea loads on a beam structure

\section{B3. Wave and current loads}

For a beam with diameter smaller than $1 / 5$ of wave length, the sea loads on the beam can be calculated by using Morison's equation (Veritas, 1993). As suggested by Veritas (1993), longitudinal component of the sea load can be neglected. Therefore, only load normal to the direction of the beam is considered. The distributed force per unit length acting normal to the beam direction due to wave and current loads can be represented as

$\mathbf{F}_{n(f \text { fluid })}\left(\mathbf{x}_{(k)}^{\prime}, t\right)=\mathbf{F}_{n(f \text { fluid }) \text {,inertia }}\left(\mathbf{x}_{(k)}^{\prime}, t\right)+\mathbf{F}_{n(f \text { fuid }), \text { drag }}\left(\mathbf{x}_{(k)}^{\prime}, t\right)$

where $\mathbf{F}_{n(f \text { fuid),inertia }}$ represents the inertia force and $\mathbf{F}_{n(f \text { fuid }) \text {,rrag }}$ represents the drag force acting at material point $k$. 
The inertia force in Eq. (B.3) can be represented as

$\mathbf{F}_{n(f \text { fluid }) \text {,inertia }}\left(\mathbf{x}_{(k)}^{\prime}, t\right)=\rho_{(\text {fluid })} \frac{\pi D^{2}}{4} C_{m} \cdot \mathbf{a}_{n(\text { fluid })}^{\prime}\left(\mathbf{x}_{(k)}^{\prime}, t\right)$

where $D$ represents the diameter of the beam, $\mathbf{a}_{n(f \text { fluid })}^{\prime}$ represents the fluid acceleration normal to the beam centre line and $C_{m}$ represents the inertia coefficient which is considered as $C_{m}=1.8$ (Faltinsen, 1993).

The drag force in Eq. (B.3) can be represented as

$\mathbf{F}_{n(\text { fluid }) \text {,drag }}\left(\mathbf{x}_{(k)}^{\prime}, t\right)=\frac{1}{2} \rho_{(w)} D C_{d} \cdot \mathbf{v}_{n(f \text { fluid })}^{\prime}\left(\mathbf{x}_{(k)}^{\prime}, t\right)\left|\mathbf{v}_{n(f \text { fluid })}^{\prime}\left(\mathbf{x}_{(k)}^{\prime}, t\right)\right|$

where $\mathbf{v}_{n(f \text { flid })}^{\prime}$ represents the fluid velocity normal to the beam centre line and $C_{d}$ represents the drag coefficient which is considered as $C_{d}=0.7$ (Faltinsen, 1993).

The normal component of velocity and acceleration can be calculated as

$\mathbf{v}_{n(\text { fluid })}^{\prime}\left(\mathbf{x}_{(k)}^{\prime}, t\right)=\mathbf{v}_{(f l u i d)}^{\prime}\left(\mathbf{x}_{(k)}^{\prime}, t\right) \cdot \sin \alpha_{\left(\mathbf{v}_{n(f \text { fuid })}^{\prime}\left(\mathbf{x}_{(k)}^{\prime}, t\right), n_{x(k)}\right)}$

$\mathbf{a}_{n(\text { fluid })}^{\prime}\left(\mathbf{x}_{(k)}^{\prime}, t\right)=\mathbf{a}_{(f l u i d)}^{\prime}\left(\mathbf{x}_{(k)}^{\prime}, t\right) \cdot \sin \alpha_{\left(\mathbf{a}_{(f \text { fuid }}^{\prime}\left(\mathbf{x}_{(k)}^{\prime}, t\right), n_{x(k)}\right)}$

where $\alpha_{\left(\mathbf{v}_{n}^{\prime}(f \text { muli })\left(\mathbf{x}_{(k)}^{\prime}, t\right), n_{x(k)}\right)}$ represents the angle between velocity vector and unit vector and $\alpha_{\left(\mathbf{a}_{(f, \mu l i d)}^{\prime}\left(\mathbf{x}_{(k)}^{\prime}, t\right), n_{x(k)}\right)}$ represents the angle between acceleration vector and unit vector as shown in Fig.

B2.

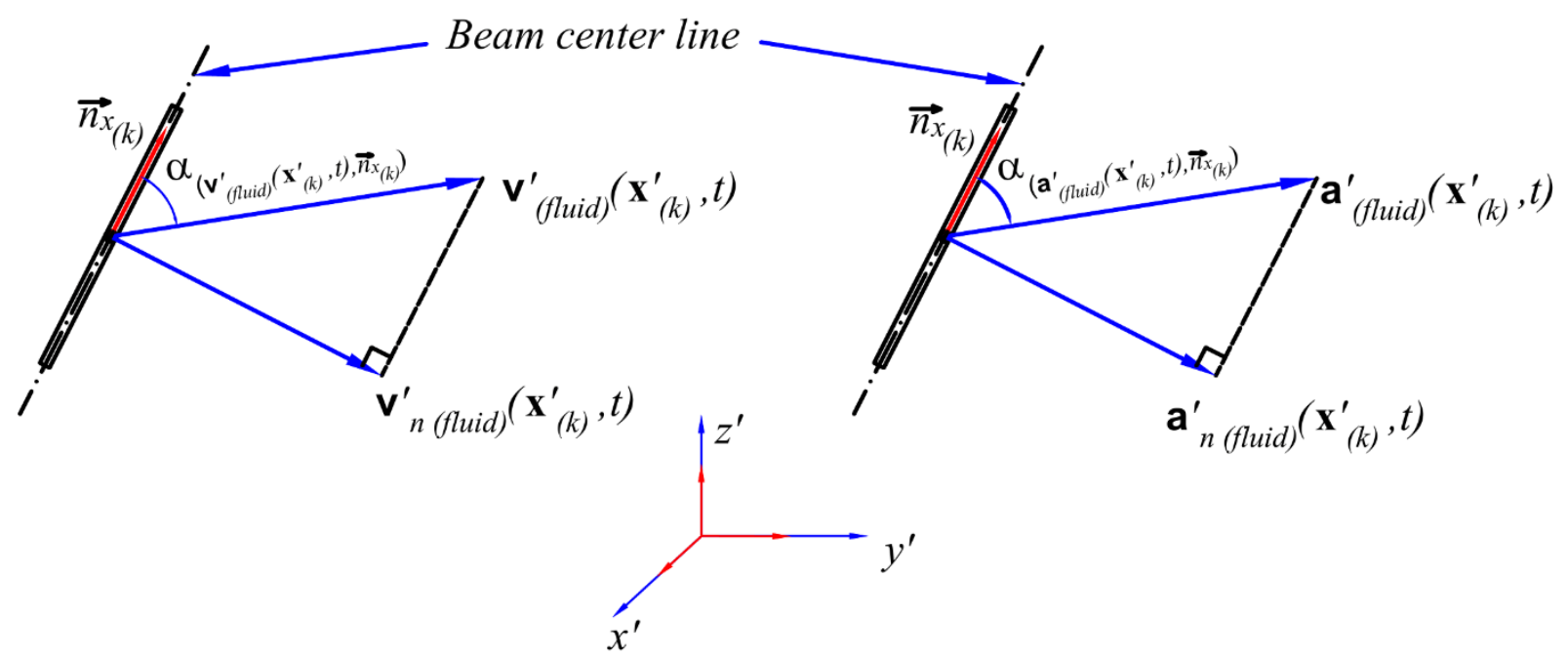

Fig. B2. Fluid velocity and acceleration components

\section{B4. Wind loads}

Similar to wave load only the component of wind force normal to beam direction is considered as shown in Fig. B1. The distributed force per unit length acting normal to the beam direction due to wind loads can be represented as

$\mathbf{F}_{n(\text { wind })}\left(\mathbf{x}_{(k)}^{\prime}, t\right)=\frac{1}{2} \rho_{(\text {air })} D C_{(\text {air })} \mathbf{v}_{n(\text { wind })}^{\prime}\left(\mathbf{x}_{(k)}^{\prime}, t\right)\left|\mathbf{v}_{n(\text { wind })}^{\prime}\left(\mathbf{x}_{(k)}^{\prime}, t\right)\right|$ 
where $\rho_{(\text {air })}$ represents the density of air, $\mathbf{v}_{n(\text { wind })}^{\prime}$ represents the components of wind velocity normal to the beam centre line and $C_{(\text {air })}$ represents the air drag coefficient.

The normal component of wind velocity can be calculated as

$\mathbf{v}_{n(\text { wind })}^{\prime}\left(\mathbf{x}_{(k)}^{\prime}, t\right)=\mathbf{v}_{(\text {wind })}^{\prime}\left(\mathbf{x}_{(k)}^{\prime}, t\right) \cdot \sin \alpha_{\left(\mathbf{v}_{n(\text { wind })}^{\prime}\left(\mathbf{x}_{(k)}^{\prime}, t\right), n_{x(k)}\right)}$

where $\alpha_{\left(\mathbf{v}_{n(\text { wind })}^{\prime}\left(\mathbf{x}_{(k)}^{\prime}, t\right), n_{x(k)}\right)}$ represents the angle between vectors $\mathbf{v}_{(\text {wind })}^{\prime}\left(\mathbf{x}_{(k)}^{\prime}, t\right)$ and $n_{x(k)}$ as shown in Fig. B3.

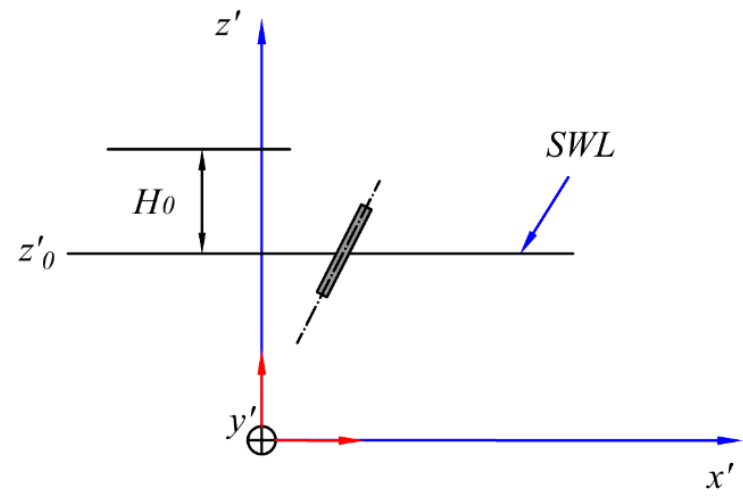

Side view

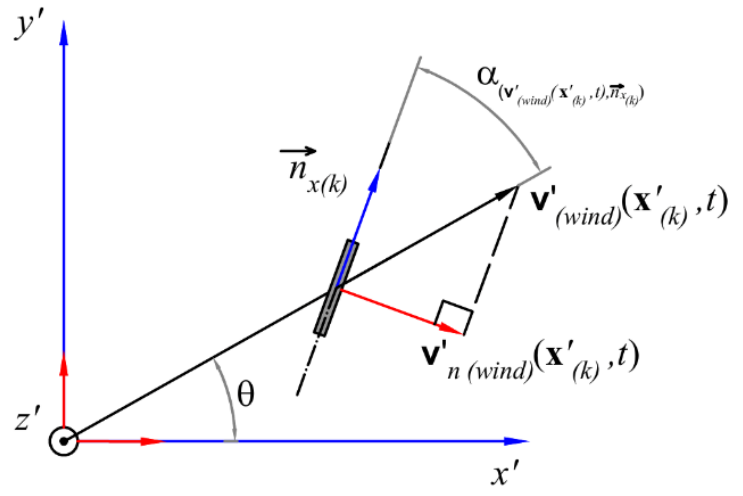

Top view

Fig. B3. Sketch of an element in a wind field (Veritas, 1993)

Air drag coefficient, $C_{(\text {air })}$ can be evaluated based on the Reynolds number of air flow as (Veritas, 1993)

$C_{(\text {air })}=\left\{\begin{array}{llr}1.2 & \text { for } & 0<\operatorname{Re} \leq 2.5 \times 10^{5} \\ 7.2426 \times 10^{10} / \mathrm{Re}^{1.9972} & \text { for } 2.5 \times 10^{5}<\operatorname{Re} \leq 3.8 \times 10^{5} \\ \operatorname{Re}^{0.2557} / 51.392 & \text { for } & 3.8 \times 10^{5}<\operatorname{Re}\end{array}\right.$

with

$\operatorname{Re}=\frac{\left|\mathbf{v}_{n(\text { wind })}^{\prime}\left(\mathbf{x}_{(k)}^{\prime}, t\right)\right| D}{v_{a}}$

and $v_{a}$ represents the viscosity coefficient for air.

In this study, the velocity formulation of wind is defined as (Veritas, 1993)

$\mathbf{v}_{(\text {wind })}^{\prime}=V_{(\text {wind })} f\left(\frac{z^{\prime}-z_{0}^{\prime}}{H_{0}}\right)^{P}\left(\mathbf{i}_{1} \cos \theta+\mathbf{i}_{2} \sin \theta\right)$

where $H_{0}$ represents the reference height, $V_{(\text {wind })}$ represents the mean wind velocity at the reference height, $f$ represents the gust factor, $P$ represents the height exponent, $z_{0}^{\prime}$ represents the position of the still water level and $\theta$ represents the angle of wind direction with the global $x^{\prime}$ axis as shown in Fig. B3. Note that in this study position of the sea water level is taken as water depth. 
The reference height $H_{0}$ is defined as the distance from sea water level to the mean wind velocity reference level (Veritas, 1993).

\section{B5. Numerical procedure for loading calculation}

The numerical procedure for loading calculation can be summarised as follows;

(1) Define geometry and other input parameters such as;

- Structural material properties, water and air properties

- Wave properties including wave height, $H$, wave period, $T$, wave direction, $\beta$, water depth, $d$, current velocity profile, $\mathbf{v}_{(\text {current })}^{\prime}$

- Wind properties including mean wind velocity, $V_{(\text {wind })}$, reference height, $H_{0}$, wind direction $\theta$, gust factor, $f$, height exponent, $P$

(2) Discretization: The model is discretized into $N_{p}$ material points.

(3) Define wave surface:

- The wave length, $L$ is calculated by using Eq. (A .6) for deep water, or Eq. (A.10) for shallow water

- The wave angular frequency, $\omega$, absolute value of wave number, $k$, two dimensional wave number, k , are calculated by using Eq. (A.5), (A.4), and (A.3), respectively.

- The wave surface is then defined by using Eq. (A1).

(4) Loop 1: Create a loop over time steps: $t=0$ to $N_{t}$ with time step size $d t$.

(4.1) Find the intersection points between the structure and the wave surface to define the part of the structure that is above water and below water surface.

(4.2) Loop 2: Create a loop over the structural material points: $k=1$ to $N_{p}$

(4.2.1) Determine wave kinematics:

- For deep water, the components of fluid velocity and acceleration located at the same position with material point $k$ is calculated by using Eq. (A.7) and (A.8), respectively.

- For shallow water, the fluid velocity and acceleration can be calculated using Eq. (A.11), and (A.12), respectively.

- The horizontal component of fluid velocity is calculated using Eq. (A.9). It is assumed that the horizontal component of fluid velocity of points located above SWL is set equal to the value of horizontal fluid velocity at SWL as shown in Fig. (A.2).

(4.2.2) The total fluid velocity and acceleration can be calculated by using Eq. (A.14)

(4.2.3) Calculate wind velocity using Eq. (B.12)

(4.2.4) Gravitational loads: calculate gravitational loads using Eq. (B.1)

(4.2.5) Buoyancy loads: If material point $k$ is below water surface, the buoyancy load is calculated by using Eq. (B.2). Otherwise, there is no buoyancy load.

(4.2.6) Wave and current loads:

- The components that normal to the beam direction of fluid velocity and acceleration can be calculated by using Eq. (B.6), and (B.7).

- The force caused by fluid is then calculated by using Eq. (B.3).

- The force acting on material point, $k$ due to fluid can be calculated by multiplying Eq. (B.3) by grid size, $\Delta x$.

(4.2.7) Wind loads:

- The normal component of wind velocity can be calculated is calculated using Eq. (B.9) 
- The Reynolds number of air flow is calculated using Eq. (B.11)

- The air drag coefficient, $C_{(a i r)}$ is calculated by using Eq. (B.10)

- The wind forces can be calculated by using Eq. (B.8). The wind force acting on current material point, $k$ can be calculated by multiplying Eq. (B.8) by grid size, $\Delta x$.

(4.2.8) Go to next material point until loop 2 finishes.

(4.2.9) Store data of loading at current time $t$ for all structural material points.

(4.3) Go to step (4.1) until loop 1 finishes.

(5) Output loading calculation for each material point at each time step.

\section{Appendix C. Adaptive Dynamic Relaxation for static and quasi-static solution}

For static and quasi-static problems, the adaptive dynamic relaxation (ADR) can be used (Kilic and Madenci, 2010). The mass vector $\mathbf{m}_{(k)}^{\prime}$ can be replaced by a mass stable vector $\mathbf{M}_{(k)}^{\prime}$, and a damping matrix $\mathbf{C}_{(k)}^{\prime}$ is added into the equation of motion given in Eq. (43) as

$$
\mathbf{M}_{(k)}^{\prime} \mathbf{u}_{(k)}^{\prime}+\mathbf{C}_{(k)}^{\prime} \mathbf{u}_{(k)}^{\prime}=\sum_{j=1} \mu_{(k)(j)} \mathbf{f}_{(k)(j)}^{\prime} V_{(j)}+\mathbf{b}_{(k)}^{\prime}
$$

According to Underwood, (1983), the right-hand side of Eq. (C.1) should be real physical values, but the left-hand side of Eq. (C.1), which includes mass stable vector and the damping matrix which can be chosen arbitrarily to get a converged solution. However, the matrix $\mathbf{M}_{(k)}^{\prime}$ is required to be a diagonal matrix. A simple way for determination of mass stable vectors in global coordinates is described as follows

$$
\mathbf{M}_{(k)}^{\prime}=\left[\begin{array}{cccccc}
M_{u_{(k)}^{\prime}}^{\prime} & 0 & 0 & 0 & 0 & 0 \\
0 & M_{v^{\prime}(k)}^{\prime} & 0 & 0 & 0 & 0 \\
0 & 0 & M_{w_{(k)}^{\prime}}^{\prime} & 0 & 0 & 0 \\
0 & 0 & 0 & M_{\theta_{x^{\prime}(k)}}^{\prime} & 0 & 0 \\
0 & 0 & 0 & 0 & M_{\theta_{y^{\prime}(k)}}^{\prime} & 0 \\
0 & 0 & 0 & 0 & 0 & M_{\theta_{z^{\prime}(k)}}^{\prime}
\end{array}\right]
$$

with

$$
\begin{aligned}
& M_{u_{(k)}^{\prime}}^{\prime}=M_{v_{(k)}^{\prime}}^{\prime}=M_{w_{(k)}^{\prime}}^{\prime}=\max \left(M_{u_{(k)}}, M_{v_{(k)}}, M_{w_{(k)}}\right) \\
& M_{\theta_{x^{\prime}(k)}}^{\prime}=M_{\theta_{y^{\prime}(k)}^{\prime}}^{\prime}=M_{\theta_{z^{\prime}(k)}}^{\prime}=\max \left(M_{\theta_{x^{\prime}(k)}}, M_{\theta_{y^{\prime}(k)}}, M_{\theta_{z_{z(k)}}}\right)
\end{aligned}
$$

where $M_{u^{\prime}(k)}^{\prime}, M_{v^{\prime}(k)}^{\prime}, M_{w_{(k)}^{\prime}}^{\prime}, M_{\theta_{x^{\prime}(k)}}^{\prime}, M_{\theta_{y^{\prime}(k)}}^{\prime}, M_{\theta_{z^{\prime}(k)}}^{\prime}$ represent the components mass stable vector corresponding to translational and rotational DOFs in the global coordinates, $M_{u_{(k)}}, M_{v_{(k)}}, M_{w_{(k)}}$, $M_{\theta_{x(k)}}, M_{\theta_{y(k)}}, M_{\theta_{z(k)}}$ represent the components of a mass stable vector in the local coordinates. The components of the mass stable vector in the local coordinates can be calculated based on PD bond constants as

$$
M_{u_{(k)}} \geq \frac{1}{4} \frac{d t^{2} A C_{a x} \delta}{d x}
$$




$$
\begin{aligned}
& M_{v_{(k)}}=M_{w_{(k)}} \geq \frac{1}{4} \frac{d t^{2} A C_{s} \delta}{d x} \\
& M_{\theta_{x(k)}} \geq \frac{1}{4} \frac{d t^{2} A C_{t} \delta}{d x} \\
& M_{\theta_{y(k)}} \geq \frac{1}{4} \frac{d t^{2} A C_{b y} \delta}{d x} \\
& M_{\theta_{z(k)}} \geq \frac{1}{4} \frac{d t^{2} A C_{b z} \delta}{d x}
\end{aligned}
$$

where $d t=1$ represent the time step for quasi-static solution (Underwood, 1983).

\section{References}

Andersen, T.L., Frigaard, P. and Burcharth, H.F., 2014. Lecture notes for the course in water wave mechanics.

Askari, E., Bobaru, F., Lehoucq, R.B., Parks, M.L., Silling, S.A. and Weckner, O., 2008. Peridynamics for multiscale materials modeling. In Journal of Physics: Conference Series (Vol. 125, No. 1, p. 012078). IOP Publishing.

Bathe, K.J., 2006. Finite element procedures. Klaus-Jurgen Bathe.

Bie, Y.H., Cui, X.Y. and Li, Z.C., 2018. A coupling approach of state-based peridynamics with node-based smoothed finite element method. Computer Methods in Applied Mechanics and Engineering, 331, pp.675-700.

Bobaru, F. and Ha, Y.D., 2011. Adaptive refinement and multiscale modeling in 2D peridynamics

Boresi, A.P., Schmidt, R.J. and Sidebottom, O.M., 1985. Advanced mechanics of materials (Vol. 6). New York et al.: Wiley.

De Meo, D., Zhu, N. and Oterkus, E., 2016. Peridynamic modeling of granular fracture in polycrystalline materials. Journal of Engineering Materials and Technology, 138(4), p.041008.

Diyaroglu, C., 2016. Peridynamics and its applications in marine structures (Doctoral dissertation, University of Strathclyde).

Diyaroglu, C., Oterkus, E., Oterkus, S. and Madenci, E., 2015. Peridynamics for bending of beams and plates with transverse shear deformation. International Journal of Solids and Structures, 69, pp.152-168.

Diyaroglu, C., Oterkus, E. and Oterkus, S., 2017a. An Euler-Bernoulli beam formulation in an ordinary state-based peridynamic framework. Mathematics and Mechanics of Solids, p.1081286517728424.

Diyaroglu, C., Oterkus, S., Oterkus, E. and Madenci, E., 2017b. Peridynamic Modeling of Diffusion by Using Finite-Element Analysis. IEEE Transactions on Components, Packaging and Manufacturing Technology, 7(11), pp.1823-1831.

Faltinsen, O., 1993. Sea loads on ships and offshore structures (Vol. 1). Cambridge university press.

Foster, J.T., Silling, S.A. and Chen, W.W., 2010. Viscoplasticity using peridynamics. International journal for numerical methods in engineering, 81(10), pp.1242-1258.

Gao, Y. and Oterkus, S., 2019. Fully coupled thermomechanical analysis of laminated composites by using ordinary state based peridynamic theory. Composite Structures, 207, pp.397-424.

Gao, Y. and Oterkus, S., 2018. Peridynamic Analysis of Marine Composites under Shock Loads by Considering Thermomechanical Coupling Effects. Journal of Marine Science and Engineering, 6(2), p.38.

Guo, J., 2002. Simple and explicit solution of wave dispersion equation. Coastal Engineering, 45(2), pp.7174.

Han, S., Lim, S., Bae, J., Hwang, Y., Lee, S., Oterkus, S., Madenci, E., Diyaroglu, C. and Oterkus, E., 2015, 
May. Equivalent acceleration assessment of JEDEC moisture sensitivity levels using peridynamics. In Electronic Components and Technology Conference (ECTC), 2015 IEEE 65th (pp. 1518-1523). IEEE.

Han, S.W., Diyaroglu, C., Oterkus, S., Madenci, E., Oterkus, E., Hwang, Y. and Seol, H., 2016, May. Peridynamic direct concentration approach by using ANSYS. In Electronic Components and Technology Conference (ECTC), 2016 IEEE 66th (pp. 544-549). IEEE.

Hu, W., Ha, Y.D. and Bobaru, F., 2012. Peridynamic model for dynamic fracture in unidirectional fiberreinforced composites. Computer Methods in Applied Mechanics and Engineering, 217, pp.247-261.

Javili, A., Morasata, R., Oterkus, E. and Oterkus, S., 2018. Peridynamics review. Mathematics and Mechanics of Solids, p.1081286518803411.

Kilic, B. and Madenci, E., 2010a. An adaptive dynamic relaxation method for quasi-static simulations using the peridynamic theory. Theoretical and Applied Fracture Mechanics, 53(3), pp.194-204.

Kilic, B. and Madenci, E., 2010b. Coupling of peridynamic theory and the finite element method. Journal of mechanics of materials and structures, 5(5), pp.707-733.

Le Mehaute, B., Divoky, D. and Lin, A., 1969. Shallow water waves a comparison of theories and experiments. In Coastal Engineering 1968 (pp. 86-107).

Le Méhauté, B., 1969. An Introduction to Hydrodynamics and Water Waves Volume II: Water Wave Theories.

Liu, W. and Hong, J.W., 2012. A coupling approach of discretized peridynamics with finite element method. Computer Methods in Applied Mechanics and Engineering, 245, pp.163-175.

Macek, R.W. and Silling, S.A., 2007. Peridynamics via finite element analysis. Finite Elements in Analysis and Design, 43(15), pp.1169-1178.

Madenci, E. and Oterkus, E., 2014. Peridynamic theory and its applications (Vol. 17). New York: Springer.

Madenci, E. and Oterkus, S., 2016a. Ordinary state-based peridynamics for plastic deformation according to von Mises yield criteria with isotropic hardening. Journal of the Mechanics and Physics of Solids, 86, pp.192-219.

Madenci, E. and Oterkus, S., 2016b. Peridynamics for Coupled Field Equations. Handbook of Peridynamic Modeling.

Madenci, E. and Oterkus, S., 2017. Ordinary state-based peridynamics for thermoviscoelastic deformation. Engineering Fracture Mechanics, 175, pp.31-45.

Mader, C.L., 2004. Numerical modeling of water waves. Crc Press.

Mitchell, J.A., 2011. A non-local, ordinary-state-based viscoelasticity model for peridynamics. Sandia National Lab Report, 8064, pp.1-28.

Silling, S.A., 2000. Reformulation of elasticity theory for discontinuities and long-range forces. Journal of the Mechanics and Physics of Solids, 48(1), pp.175-209.

Silling, S.A., Epton, M., Weckner, O., Xu, J. and Askari, E., 2007. Peridynamic states and constitutive modeling. Journal of Elasticity, 88(2), pp.151-184.

Silling, S.A. and Askari, E., 2005. A meshfree method based on the peridynamic model of solid mechanics. Computers \& structures, 83(17-18), pp.1526-1535.

Silling, S.A., Zimmermann, M. and Abeyaratne, R., 2003. Deformation of a peridynamic bar. Journal of Elasticity, 73(1-3), pp.173-190.

Stephen, N.G., 1981. Considerations on second order beam theories. International Journal of Solids and Structures, 17(3), pp.325-333.

O'Grady, J. and Foster, J., 2014. Peridynamic beams: a non-ordinary, state-based model. International Journal of Solids and Structures, 51(18), pp.3177-3183.

Oterkus, E., 2010. Peridynamic theory for modeling three-dimensional damage growth in metallic and composite structures.

Oterkus, E. and Madenci, E., 2012. Peridynamic theory for damage initiation and growth in composite laminate. In Key Engineering Materials (Vol. 488, pp. 355-358). Trans Tech Publications.

Oterkus, E., Madenci, E., Weckner, O., Silling, S., Bogert, P. and Tessler, A., 2012. Combined finite element and peridynamic analyses for predicting failure in a stiffened composite curved panel with a entral slot. Composite Structures, 94(3), pp.839-850. 
Oterkus, S., 2015. Peridynamics for the solution of multiphysics problems (Doctoral dissertation, The University of Arizona).

Oterkus, S. and Madenci, E., 2015. Peridynamics for antiplane shear and torsional deformations. Journal of Mechanics of Materials and Structures, 10(2), pp.167-193.

Oterkus, S. and Madenci, E., 2017. Peridynamic modeling of fuel pellet cracking. Engineering Fracture Mechanics, 176, pp.23-37.

Oterkus, S., Madenci, E. and Oterkus, E., 2017. Fully coupled poroelastic peridynamic formulation for fluid-filled fractures. Engineering Geology, 225, pp.19-28.

Timoshenko, S. P., \& Goodier, J. N., 1970. Theory of elasticity (Vol. 3, pp. 403-408). London: McGrawHill, New York.

Underwood, P., 1986. Dynamic relaxation. Computational method for transient analysis, 1, pp.245-263.

Veritas, D.N., 1993. WAJAC--Wave and Current Loads on Fixed Rigid Frame Structures. DNV SESAM AS. Version, 5(02).

Villavicencio, R. and Soares, C.G., 2012. Numerical plastic response and failure of a pre-notched transversely impacted beam. Ships and Offshore Structures, 7(4), pp.417-429.

Yang, Z., Oterkus, E., Nguyen, C.T. and Oterkus, S., 2018. Implementation of peridynamic beam and plate formulations in finite element framework. Continuum Mechanics and Thermodynamics, pp.1-15. 